Article

\title{
Critical Assessment of Theoretical Calculations of Atomic Structure and Transition Probabilities: An Experimenter's View
}

\section{Elmar Träbert ${ }^{1,2}$}

${ }^{1}$ AIRUB, Fakultät für Physik und Astronomie, Ruhr-Universität Bochum, 44780 Bochum, Germany; E-Mail: traebert@astro.rub.de; Tel.: +49-234-3223451

${ }^{2}$ Physics Division, Physical and Life Sciences, Lawrence Livermore National Laboratory, Livermore, CA 94550, USA

Received: 25 January 2014; in revised form: 5 March 2014 / Accepted: 5 March 2014 / Published: 19 March 2014

\begin{abstract}
The interpretation of atomic observations by theory and the testing of computational predictions by experiment are interactive processes. It is necessary to gain experience with "the other side" before claims of achievement can be validated and judged. The discussion covers some general problems in the field as well as many specific examples, mostly organized by isoelectronic sequence, of what level of accuracy recently has been reached or which atomic structure or level lifetime problem needs more attention.
\end{abstract}

Keywords: atomic spectra; lifetimes; fine structure

Classification: PACS 32.30.-r, 32.70.Cs, 32.10.Fn

\section{Introduction}

Let me refer to higher authority for guidance in the task prescribed in the title. Burkhard Fricke, Professor of Theoretical Physics at Kassel, some time vice president of his university, recalls advice by his elders as "If theory and experiment disagree, work on theory; if they agree, improve the experiment." Alan Hibbert, professor in the Department of Applied Mathematics and Theoretical Physics at Queens University Belfast and sometime Dean also of the Faculty of Theology, would add a caution to this: "If theory and experiment agree, be aware of the possibility that both may be wrong." Right. Moreover, if one considers another research practicality, one may suffer the consequences "if theory and experiment agree, you won't get funding for further research." 
My aim in this article is a discussion of what $\mathrm{I}$ as an experimenter expect from theory and atomic structure computations, how I try to assess the quality of the work, and what experiment can or can't do towards a critical validation process of such calculations. After all, experiments and experimenters come in many flavors, too. We all make mistakes, and maybe we can learn from some of them that we have recognized by now. In this context, I dare to digress into anecdotal evidence of various problems in the interplay of atomic structure theory and experiment, into human and technical sources of error and uncertainty, before turning to a more systematic discussion of data on specific isoelectronic sequences. However, I will not present an ultimate recipe for assessing calculated data. Both, experiment and theory, are intertwined in the scientific process, and progress may involve a new perspective, if not always a paradigm shift. More than three decades ago, when I joined Josh Silver's group at Oxford, we wanted to do spectroscopy on two-electron systems with measurements that ought to be good enough to test the smallish QED contributions. We thought QED to be important, and we wanted to challenge theory (and theoreticians). When the agreement with theory turned out only moderately good, we wanted to blame the quality of the QED calculations, but were informed by the theorist A. M. Ermolaev that the theory authors actually believed them to be highly accurate. Those people conceded that the shortcomings lay in the quantum mechanical treatment of the two-electron system-a topic seemingly much less fancy than QED, but nonetheless essential and fundamental. This perspective has been corroborated over and over again since. Thus it is not lofty "theory" that experiment is testing, but computer implementations of many-body quantum mechanics, relativistic wave functions, nuclear magnetic field distributions, multipole expansions of the radiation field, and so on-in a package that combines some of almost everything. The more detail is to be treated, the more unwieldy become the programs, and the fewer cases are being calculated for a given amount of grant money. Any practical atomic structure computation represents a compromise, just as any measurement is a result achieved with limited insight and means. Yet some turn out better than others and need to be recovered from the chaff of computations and whatever unstructured data. Maybe one could develop a feeling for quality?

It is difficult to draw a line at which to begin or stop discussing data and developments, be it on the grounds of progress being made or of data quality. Various good people have written useful surveys of the material, and there also are a number of my own discursive listings of atomic lifetime and spectral data. There is no need to replay such presentations for completeness of content here. I have been guided in the field by some experts such as Lorenzo J. ("Larry") Curtis and Indrek Martinson (for samples of their reviews and systematizations see [1-4]). Curtis and Martinson have always been extremely polite when discussing other peoples' work, even when finding fault as the result of their own scrutiny. My language is more blunt, especially when I discuss bad science, but it is not intended to exceed the educative. Curtis and Martinson have worked — among other techniques—with beam-foil spectroscopy, from the very early days of the field, and they have developed many of the crucial insights that helped to obtain reliable wavelength and lifetime data. I have come to the same field later and thus had to find my niche by interacting with established groups elsewhere, by working on heavier ions, shorter and longer atomic lifetimes, peculiarities of time-resolved spectroscopy, and so on. In a later period I then learned how to operate ion traps and to exploit a heavy-ion storage ring and electron beam ion traps. My views are, of course, trained and tainted by the experience. My report will largely bypass classical spectroscopy, because much of that has happened before my time, has either been done at national 
standards laboratories or long since been critically evaluated there. The task put to me is not to recount "how we got there", but to assess the situation now, how to find an orientation and how to possibly move onward. Also, I am not trying to evaluate computational techniques and programs. There is a case (recalled below) in which notionally the same technique has been employed by members of an erstwhile collaboration; one part of the collaboration has proceeded to much better results within a year, while the other collaborators stuck to the poorer version even a year after that. Evidently one should not blame the technique per se, but the executors. As a user of computational results, I am rather more interested in whether the results are reliable and useful because they are tied to experimental data or whether they have been obtained $a b$ initio and thus represent our theoretical understanding of atomic structure - at the risk of a possibly poorer match with the data.

I am grateful to the editors for letting me combine atomic energy levels and transition rates in the discussion. There is the Ritz variation principle that states how any imperfection in the wave function of the lowest state of a given symmetry will result in a calculated energy higher than in nature/truth. For decades this insight has fired a competition for reaching a lower value for the computed energy of the ground state of the He atom, and the guidance has resulted in excellent achievements. Moreover, in the calculation of level energies theory often separates the wave functions into angular and radial parts. For a long time, orthogonality of the radial wave functions has been considered essential, but nowadays even nonorthogonal operators are employed. There now are some extremely accurate level computations, but why is theory so much less reliable with transition rates?

Firstly, the transition dipole operator er for electric dipole (E1) transitions explicitly contains the radial part of the wave function, and this forces the problem of orthogonality to the light, while at the same time there is no variational principle available that defines a boundary condition for guidance.

Secondly, in a single-configuration picture the transitions between fine structure levels or between the ground configuration levels of a multi-electron system (all of the same parity, by definition) are not E1 transitions and these rates do not depend on an operator with radial wave functions. However, the single-configuration picture is simplistic, and deeper investigations by theory as well as accurate experiments show the need for a multi-configuration description and thus for taking care of considerable complexity. Here the transition rate depends on a higher power of the transition energy, and it is difficult to calculate these particular energy differences with a sufficient accuracy.

Thirdly, intercombination transitions are usually modeled as being mediated by spin-orbit coupling and the multiplet mixing of very few levels. The Breit operator, however, ought to be represented by a series expansion, but in practice this is usually truncated after the first term (!), because it is so computationally challenging. Combining that with the need to calculate the influence of all levels with the same parity and total angular momentum, it becomes clear that there may be more problems lurking in dark corners than have been recognized so far. The contributions listed are intertwined; in the discussion below there are cases that are dominated by one or another of these problems. It should come as no surprise that not all of them have been solved yet. It takes comparisons between nature/experiment and theory/computation to find out about the amount of detail that needs to be explored. 


\subsection{Codes}

Among the many codes used in atomic structure computations there are veterans and newcomers, well-trained packages in new disguises, re-packaged well-proven standard parts, and more. Look into the codes and find contributions from many authors, some of them vintage, some newly phrased or added. Debugging new code is tedious and takes a lot of experience and judgement to recognize problems in the product. Some of the codes alluded to below and some of their exemplary developers-cum-users (as far as I know) are:

CIV3 (non-relativistic, with partial relativistic corrections) Configuration Interaction Version 3, developed by A. Hibbert and his group (including many foreign students) at Belfast,

HULLAC Hebrew University Lawrence Livermore Atomic Code, developed by M. Klapisch in Israel and various colleagues as well as many people at LLNL,

MCHF Multi-Configuration Hartree-Fock (non-relativistic, with relativistic corrections, etc.), a classic development over decades by the likes of C. Froese Fischer and her team when she was at Vanderbilt,

MCDF Multi-Configuration Dirac-Fock and MCDHF Multi-Configuration Dirac-Hartree-Fock (relativistic wave functions), with original program versions developed by J. P. Desclaux and P. Indelicato at Paris and by I. P. Grant and his team at Oxford (generic Grant code) and with additional development by C. Froese Fischer and collaborators, among them G. Tachiev, S. Fritzsche and P. Jönsson, (GRASP92, GRASP2K codes and various derivatives),

MCRRPA Multi-Configuration Relativistic Random-Phase Approximation, used by various people, notably by K.-N. Huang at Taipei,

MBPT Many-Body Perturbation Theory and RMBPT Relativistic MBPT, much pushed towards quality by W. R. Johnson, S. A. Blundell, and U. I. Safronova at Notre Dame,

RCI Relativistic Configuration Interaction, practiced and pushed to remarkable accuracy by M. H. Chen and K. T. Cheng at Livermore,

MR-MP Multi-Reference Møller-Plesset, developed by Y. Ishikawa and group (for atomic structure computations: K. Koc, M. J. Vilkas, J. López Encarnación, J. A. Santana, F. Diaz) at San Juan, Puerto Rico,

FAC Flexible Atomic Code, developed for speed, utility, extensive laptop use and collisional-radiative modeling by M. F. Gu when at Stanford, Livermore, Columbia, and Berkeley,

PCFI Partitioned Correlation Function Interaction, a recent appearance aiming at high accuracy in low charge ions, see recent papers by S. Verdebout, P. Rynkun, P. Jönsson, M. Godefroid, G. Gaigalas, and C. Froese Fischer.

There also are updates and improvements of venerable codes such as the Cowan code and its derivatives, HXR, Autostructure, Superstructure, Atom, and so on. Talking about codes, their quality and possible shortcomings, does not befit me as an experimenter who rarely, if ever, runs such a code. Atomic structure theory is a highly developed field. A real (and different) challenge lies in the proper coding for computation, as well as in the instructions for proper use. The results need to be tested by 
quality experiments (that is where I see my role). In many cases the comparison of computation and measurement will point out a need of further debugging the codes and/or to better experiments.

\section{Historical Remarks}

Here is a nasty remark from me as an experimenter right at the start: One ought to distinguish theory and computation. Not everybody running an atomic structure code qualifies as a theorist. However, the words "doing theory" are frequently used for employing somebody else's programs, and I am not singlehandedly able to abolish that custom. During the Cold War it was said that the Soviet scientists - for lack of computers-did theory, while their Western counterparts ran computers. This statement surmises a development of mathematical formalisms for the explanation of physical phenomena on one side vs. the search for numerical recipes and "mechanical" number crunching on the other. The contrast is certainly overstated, and both sides are needed, but similar biases crop up in other contexts. While the economic situation in the former Eastern bloc countries is still poor enough not to support all good scientists on location there, quite a number have found employment in the West. Thus the Western countries reap the fruits of a different scientific education path, and Eastern-born scientists have joined the number crunching cottage industry, as has the younger Chinese generation.

When atomic structure codes were developed (mostly, but not exclusively, in the West-see the ATOM code, which is based on $1 / Z$ expansions), aspiring young scientists from developing countries would go there and learn how to use the programs. Some of the young scientists grasped the underlying physics as well as the need to push out the frontier of knowledge and insight. Others were happy to obtain a copy of the program they had worked with, persuaded their home department of physics to purchase a personal computer for them (much more affordable than the equipment and running costs for an experimental laboratory), and began running the same program on the same problems as before, maybe changing the nuclear charge or the range of principal quantum numbers covered. Yes, I have seen quite a number of manuscripts submitted to learned journals in which the physics context was still at the state of thirty years earlier, most of the references were equally dated, and the results were in agreement with what other people had obtained using the Cowan code thirty years earlier (or using CIV3 not long thereafter). No, this problem is not restricted to theorists, I have also seen it with experimenters. I consider theory (in the present context atomic structure theory) as intellectually demanding. I see the quest for a valid implementation of a workable description of physical phenomena into numerical codes also as demanding (and code debugging has to go on forever in that work as well as in experiments). However, I am much less impressed if what I see is the application of somebody else's program suite to yet another case of the same ilk, the umpteenth paper on Mg-like or Al-like ions (with up to 92 elements plus the transuranes to choose from, one by one), and minute changes in the dressing. I have seen gross misunderstandings of basic quantum physics by a (theory!) coauthor of a long string of computed papers. It would be better (and save many trees and hi-tech resources) to have a code that can be run by nonspecialists on demand, if and when such numbers are actually needed. Hence I see not everything published from atomic structure computations as a product of theory or by a theoretician. In fact, both from experimenters and theoreticians I have seen manuscripts of authors who believed in their computed results (from Western programs, so they must be good?), but showed a profound lack 
of understanding the principles of atomic structure. Chance coincidences of measured and calculated wavelengths were assumed as proof of identity and classification in the spectra of foil-excited ion beams or of an ECR plasma discharge. The authors clearly disregarded that with non-selective excitation the complete spectrum of each ion charge state must be excited, not just a single line here and there, and that a spectral data analysis without attention to a sensible charge state distribution is moot. Some of that work originated in China (which I would consider as a product of authors low on the learning curve, but on their way up), and some (among other places) from a particular university physics department in the Western United States (where after many such occurrences I doubt that their learning curve was pointing up).

One major topic of my discussions will be atomic lifetime calculations and measurements. However, there is no need to repeat too much of the information discussed in earlier reviews [5-13] where also large numbers of references lead to piles of data and to explanations how to collect them. Many significant phenomena can be discussed with four-electron ions, and therefore I am presenting what I want to say in two locations, in the general section here and in a more technical one later.

\subsection{Be-Like Ions}

Be-like ions with the ground configuration $1 s^{2} 2 s^{2}{ }^{1} S_{0}$ may seem not much more complex than a $\mathrm{He}$ atom, as long as the $1 \mathrm{~s}^{2}$ core is not opened. However, there are displaced terms in the $n=2$ shell (configurations $2 \mathrm{~s} 2 \mathrm{p}$ and $2 \mathrm{p}^{2}$ ), and hence transitions of a fair variety (E1, spin-forbidden E1, M1, E2, hyperfine induced decays, etc.) can occur. In contrast to He-like ions in which the $\Delta n>0$ resonance transitions are closely related to resonance transitions in $\mathrm{H}$-like ions (which can be computed accurately), the dominant transitions in Be-like ions are $\Delta n=0$ transitions which are less easily treated by computation. Hence Be-like ions offer a laboratory for testing computational approaches against experimental data some of which challenge technology. For example, the resonance level $\left(2 \mathrm{~s} 2 \mathrm{p}{ }^{1} \mathrm{P}_{1}^{\mathrm{o}}\right)$ lifetime is rather short and dips into the picosecond range for ions beyond $Z=4$. Such lifetimes are routinely measured by beam-foil spectroscopy (see [13]), but a naively obtained apparent lifetime result taken at face value may be severely misleading, if the upper level is not selectively excited, but is suffering from cascade repopulation. This problem and its treatment (by measuring the decay curves of the cascades and performing a correlated analysis, for example in the ANDC scheme [14-17], or by employing a cascade model fit) have been discussed repeatedly [17-21] and need not be replayed here beyond mentioning their existence (see discussion below). The $2 \mathrm{~s}^{2}{ }^{1} \mathrm{~S}_{0}-2 \mathrm{~s} 2 \mathrm{p}{ }^{3} \mathrm{P}_{1}^{\mathrm{o}}$ intercombination decay rate, in contrast, is several orders of magnitude smaller than the resonance decay rate and relates (for low- $Z$ ions) to level lifetimes in the second to millisecond range. This is way too long for classical beam-foil spectroscopy, and ion trapping techniques are a necessity, with their own evolutional progress by trial and error, failure and insight. These two extreme cases provide a useful set of stepping stones for the discussion in the present context.

\subsubsection{Resonance Transition Rate}

The measurement of the resonance transition rate in Be-like ions has been a cornerstone project of beam-foil spectroscopy. When the experimental results did not match those of perceived-to-be-good 
computations, however, the discrepancy was used by some people to disregard the experimental technique and to claim (even some of the earlier proponents of the technique did so) that beam-foil spectroscopy was generally unable to produce reliable lifetime data. This impression, though formed by a lack of insight, has stuck, not the least because it has been repeated many times in authoritative conference presentations by a few key representatives from NIST and their data base project. On the experimental side, even several decades after the proper procedure of taking cascades into account had been developed, there have been publications of data evidently not involving the cascade corrections that are particularly important in Be-like ions. The cascades are so important in Be-like ions (and correspondingly in Mg- or Zn-like ions), because there are $2 \mathrm{p}^{2}{ }^{1} \mathrm{~S}_{0}$ and ${ }^{1} \mathrm{D}_{2}$ levels in the same shell as the $2 \mathrm{~s} 2 \mathrm{p}{ }^{1} \mathrm{P}_{1}^{\mathrm{o}}$ resonance level, which are easily populated in the interaction of fast ions passing through a thin foil (solid matter). The $2 \mathrm{p}^{2}{ }^{1} \mathrm{D}_{2}$ level is longer lived than the resonance level by a factor of about 4 to 5 (at low $Z$ ) and the corresponding cascade is easily recognized and treated. In contrast, the $2 \mathrm{p}^{2}{ }^{1} \mathrm{~S}_{0}$ level lifetime differs only by some $20 \%$ from that of the resonance level, for many ions along the isoelectronic sequence. This causes a growing-in cascade which, when not recognized, results in a seeming lengthening of the resonance level lifetime by some 30\%. Reistad and Martinson [22] and the present author [23] have analyzed the literature data on the members of the isoelectronic sequence and demonstrated the wide scatter of the uncorrected data as well as the isoelectronic consistency of all such lifetime data that had been evaluated with cascade-correction techniques. The isoelectronic trend of the line strength ([24] in a formulation that follows Hylleraas' expansion of basic atomic entities [25,26]) thus established is reliable to within, say, 3\% (and incidentally agrees with good quality theory). This is not bad for a system that critics have claimed to prove the unreliability of lifetime measurements by beam-foil spectroscopy.

What the experience actually does prove seems to be the fact that a fully automated analysis of decay curves can have grossly misleading results, because it disregards the information that is available from atomic structure knowledge for the system studied. No engineer would construct a bridge without reviewing the terrain and the ground, but there have been physicists who dreamed of "completely unbiased (by human interference) analyses of multi-exponential decay curves". Already decades ago Curtis and others have repeatedly demonstrated under which peculiar circumstances naive analyses of such multi-decay curve data may or even must fail, but there is no conceptual obstacle to any such measurement and meaningful evaluation once the underlying atomic structure is recognized and ANDC procedures or related modeling techniques are applied. Of course, the simplest cure for most of these problems would be the selective excitation of a given level. Unfortunately, lasers that produce multiply ionized atoms and also reach an arbitrary level of interest to modify its population are beyond technical reach so far. Let us see in the discussion below where we get with the tools we have.

Because of the aforementioned cascade correction problem and the many early "wrong" lifetime results, beam-foil researchers have mostly become overly cautious (there also is a fraction that persistently overstates the quality of their results) with error estimates for lifetime measurements. This is clearly apparent in the Be sequence. Träbert and Curtis [21] plot the resonance level lifetime (as a scaled line strength, that is, after removing the wavelength as an easily predictable or well-measured entity) and an isoelectronic trend (based on one of Curtis' many systematizations, avoiding atomic structure computations). Figure 1 shows the same situation in a comparison of experimental lifetime data with 
theory. The scatter of the data around the mean is clearly smaller than the typical individual $10 \%$ error bar. This reflects the caution of the experimenters (or maybe only a hand-waving estimate of errors by order of magnitude) as well as the good internal consistency of the data set (once the poor evaluations are discarded). Unfortunately, 3\% are an achievement on one hand, but the community would like to have even better numbers to test theory, which in this case has been claimed to be good to $\pm 100 \mathrm{ppm}$ [27].

Figure 1. Lifetime data on the $2 \mathrm{~s} 2 \mathrm{p}{ }^{1} \mathrm{P}_{1}^{o}$ level, the lowest excited singlet level in Be-like ions. The data are shown by their deviation from the lifetime predictions in the Multi-Configuration Hartree-Fock/Multi-Configuration Dirac-Hartree-Fock (MCHF/MCDHF) collection data base provided by C. Froese Fischer and G. Tachiev (the horizontal line) [28]. Also shown (broken line) is the result of a fit to the experimental data [29]. For some elements there have been several measurements, of which only the result of best quality (judged by the author) has been selected. For Be there are three data entries (the most accurate ones), and open questions remain (see text).

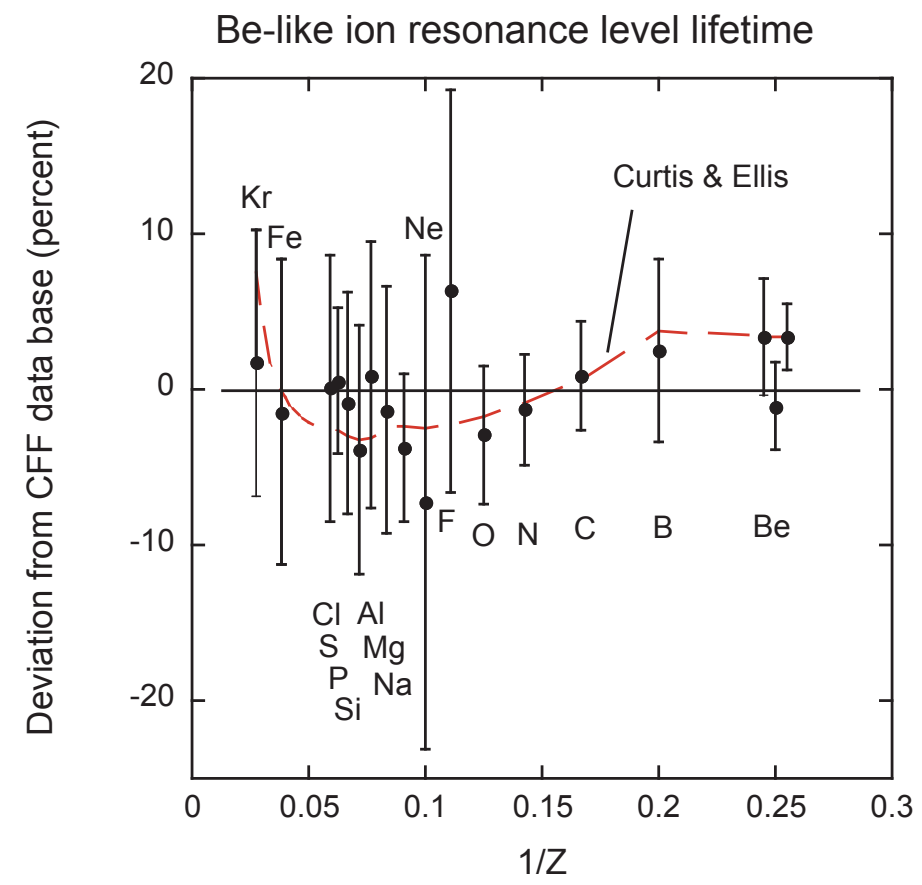

Computations of neutral atoms are particularly challenging, and, indeed, neutral $\mathrm{Be}^{0}$ has been declared to be of interest as a benchmark. There are beam-foil lifetime results from early days by Martinson, Gaupp and Curtis [30] and much later ones by Irving et al. [31], and they differ by some $4.5 \%$. Since the early data point predates the development of the ANDC cascade correction technique, one would assume that the newer data point benefits from the better insight and must be better. Because the new team included Indrek Martinson and Larry Curtis from the first measurement, one may safely assume that all technical knowledge accumulated in the community was taken care of. However, neither the old nor the newer result differs severely from the theoretical results or the aforementioned isoelectronic trend, but at about $2 \%$ the remaining deviation from the latest computations is not satisfactory. This overall reproducibility has been explained by the fact that the aforementioned strong cascade that plagues the ions in the Be isoelectronic sequence does not matter in the neutral atom, because there the cascading level would lie above the ionization limit and preferentially decay by 
autoionization. However, it is an open question what fraction of the decay is taken by autoionization, and whether the "surviving" decay branch might still cause a significant shift of the apparent lifetime of the resonance level. To me it is surprising that a system in which the recognized dominant source of systematic error (that particular cascade) is claimed to be absent should yield two lifetime results that are so different, from experiments using the same technique.

Well, I have run lifetime measurements on Na-like ions of various elements, and among those of $\mathrm{Ca}$ [32], some results differed significantly from the isoelectronic trend and from theory predictions that were beyond doubt. Although seasoned beam-foil colleagues from various countries tried their individual pet evaluation techniques, in the end we all agreed on the same numbers that differed from prediction-yet we stated in our publication that we recognized the difference and could not explain it. We do not think that theory needs to be modified, because we think the computations on Na-like ions are more reliable than a single measurement (which was repeated, of course, but did not change in result), even as that was done at the technically most suitable accelerator facility (at Bochum). However, an Asian theory colleague then suggested that theory and experiment would agree better on the lifetime, if one assumed that not all radiative decay channels mattered. He tried to sell this idea to various experts who all told him off, and he tried to have a manuscript with such anti-quantum mechanical fallacy published; as far as I know he failed in the attempt, because different journals sometimes turn to the same experts. The other measurements on the same $\mathrm{Na}$ isoelectronic sequence do not show such a deviation from the prescribed trend [33-36]. This leaves at least two explanations; firstly, from a statistical view, there "have to be" outlier results, which we can't always explain. Alternatively, since (negative) Ca ion beams (for a tandem accelerator) are difficult to produce in an ion source, the ion beam current was relatively low and thus a sufficiently high signal rate required observations through wider spectrometer slits which encompass poorer spectral resolution and enhance the likelihood of spectral blends (none has been recognized and identified yet) which might affect the decay curves. So much for the unsolved riddle of $\mathrm{Ca} \mathrm{X}$.

What may have also been at play in Be I could be an unconscious need to have a different result from before, because the new effort was more involved. A new evaluational result may therefore have received more credit than a confirmation of the old number. Why this unsubstantiated conjecture? When Schnabel and Kock [37] tried a different experimental technique, laser-induced fluorescence, their lifetime result incidentally corroborated the earlier beam-foil number rather than the newer and purportedly better one. The laser technique, however, is not necessarily guaranteed to yield correct results. A lifetime near $1 \mathrm{~ns}$ is short for producing (visible) laser light pulses at a selected wavelength. (Yes, sure, nowadays the attosecond range has been reached, but this is not practical nor sensible for many atomic physics experiments in which valence electrons populate levels that have mean lives in the nanosecond range.) The shape of the laser pulse in the time and wavelength domains needed to be modeled, and also magnetic sublevels played a role via quantum beats (a consequence of coherent excitation of nondegenerate levels). A laser experiment that aims for selective level population therefore is not necessarily as clean as one would hope for. I would not be surprised to learn that eventually experiment might find a result in the middle between those three results mentioned, which would be close to more recent computations (see discussion in [38]), but what is needed is also a smaller experimental uncertainty. 
Verdebout et al. [38] have used the resonance line of Be I as a test-case of their recently developed PCFI approach. Their theoretical oscillator strength, $f=1.375(1)$, is close to various other recent theoretical results. So, whatever experimental value is taken for comparison, the theory-experiment mismatch is around $2 \%-3 \%$, much worse than the theoretical uncertainty estimate. However, isoelectronically smoothed representations of the line strength and similar averaging procedures recounted and discussed by Irving et al. [31] pass through the region of overlap of the experimental error bars for Be I. Maybe for now the experimental results should be represented by their weighted average. (The neutral members of some other isoelectronic sequences, especially Ne I, suffer from much worse discrepancies among their experimental lifetime data - see below.)

Evidently there is still room for further experimental work on a strong and fully allowed E1 transition in a four-electron atom. This puzzling situation calls for an experiment that is independent of environmental density (beam-foil is a high-density excitation, low-density observation technique) and that comes close to selective excitation in a low-density environment, perhaps by synchrotron radiation. Beryllium, however, is toxic when in the form of breathable dust, and it would need to be dispersed as atoms (a hollow cathode discharge was used in the Schnabel and Kock work) or clusters, a clearly health-hazardous situation which one tries to avoid (not least when cleaning the apparatus after the experiment or disposing of Be traces safely). It is much easier to call for new and better measurements than to actually do them.

\subsubsection{Intercombination Transition Rate}

At the risk that the reader might see this report as full of unsubstantiated ranting, let me discuss an example in which both, theory and experiment, show ample blundering (in hindsight). Some learning theory claims that we (only) learn from our own mistakes. Help me show them wrong by learning also from the mistakes of others (at least occasionally)! The $2 \mathrm{~s}^{2}{ }^{1} \mathrm{~S}_{0}-2 \mathrm{~s} 2 \mathrm{p}{ }^{3} \mathrm{P}_{1}^{\mathrm{o}}$ intercombination transition

in Be-like ions such as $\mathrm{C}^{2+}$ (spectrum C III) is of fundamental as well as of astrophysical interest, and the calculation of the transition rate has drawn the interest of more than 30 authors or teams of authors over the years, plus more again on four-electron ions of the neighboring elements in the sequence. In the grand picture (see Figure 2, which is based on an earlier version [6]), the predictions from the 1960 s to the 1990 s scattered by $\pm 30 \%$ around a mean near $100 \mathrm{~s}^{-1}$, earning the transition rate a low accuracy rating in the Wiese tables [39]. The level lifetime $\tau$ of a level with an unbranched decay is the inverse of the transition rate $A$. In 1983, a lifetime measurement at the Harvard-Smithsonian Center for Astrophysics (CfA) employed a radiofrequency (Paul) ion trap and reported a decay rate of $100 \pm 20 \mathrm{~s}^{-1}$ at a conference [40], followed up a year later with a rate some $25 \%$ lower [41,42], and another 9 years later by a rate higher than the first one by some $20 \%$ [43]. The error bars of the purportedly improved data left a gap in the middle (where the first report had placed the result). This part of the story might reflect progress with the experiment, and thus would seem laudable. Also, from a laboratory with quite some reputation, one might expect a sound explanation of the technical progress involved, but none was given in the publications. However, I have worked in the same laboratory years later and was told details that are not mentioned in the publications. I was told by bystanders that there was not an original data set later superseded by improved measurements, but that all three publications were based on one single measurement campaign; the different results apparently stemmed from different interpretations or 
rather from shifting assumptions whether a single or two exponentials should be used in fits to the data, and whether - if using two components - the fast or the slow one should be considered the right one. Such a discussion would have befitted the publications, but it might also have limited the appeal of the work to referees and readers as a source of reliable data (with a $6 \%$ error bar in the third installment). In fact, this last data point was adopted in the new issue of the famous Wiese tables [44] at a time when the 'definitive' CfA result was already experimentally and theoretically superseded by much more accurate results - which lay smack in the middle, where the two "improved" CfA-results had left a gap (see below). To me, this is a case of misjudgement (and misinformation) by overly eager experimenters (possibly fueled by Harvard's self-image). Let us now put some blame also on theorists.

Figure 2. Intercombination transition rate in C III (adopted from Figure 4 in [6]). The timeline combines calculated $(\mathrm{T})$ and measured (E) results and is discussed in the text. The publications in this incomplete listing are identified by authors and year. The uncertainty of the 1997 measurement by Doerfert et al. is as small as the center of the cross mark. Note that a number of recent computations also bear error estimates.

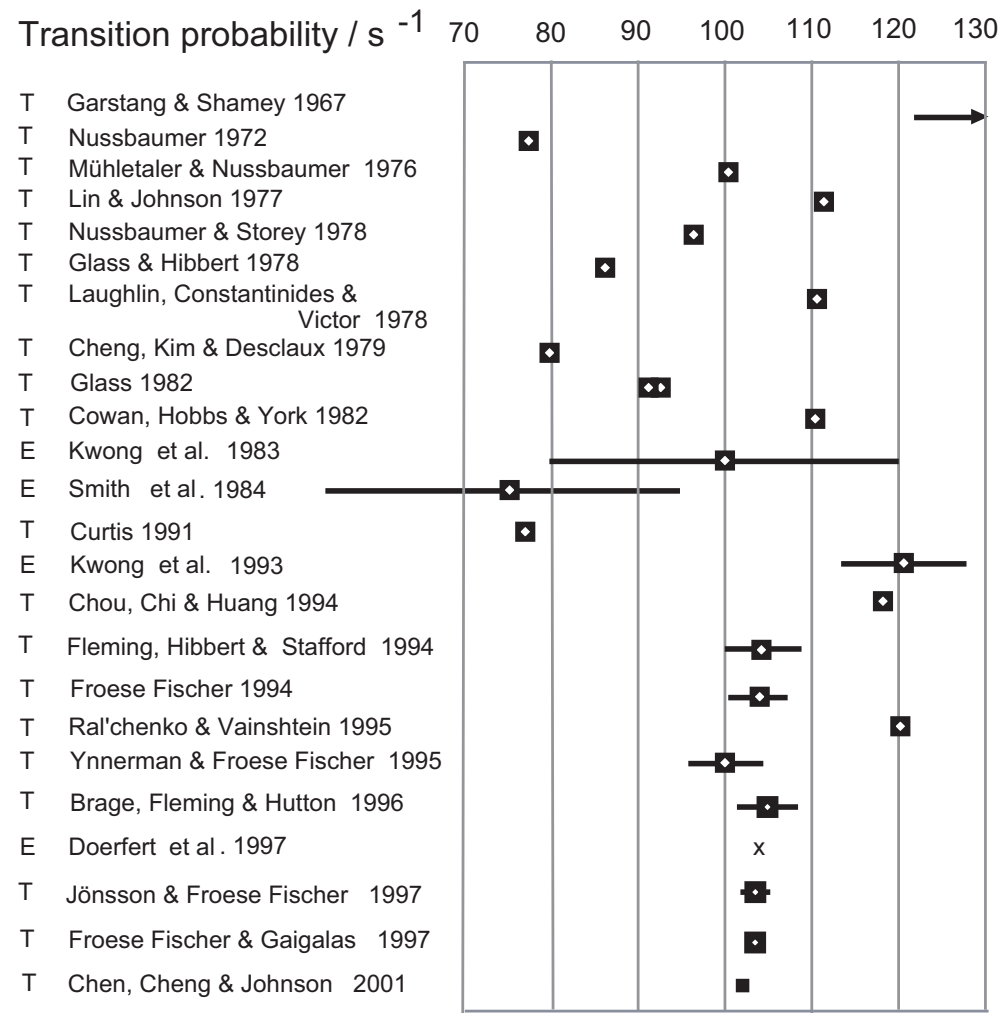

In Figure 2, a 1991 data entry by L. J. Curtis [24] follows the 1984 experimental data reported by Smith et al. [41,42] at CfA. This is not a calculation per se, but Curtis had assumed the CfA data to be reliable and thus used them as an anchor in his semi-empirical analysis of the isoelectronic sequence. Hence the isoelectronic trend he derived (a worthy exercise to bridge the wide gaps left by too few hard data) was flawed at low values of the nuclear charge $Z$, because the experimental reference data were mistaken. The next (and clearly different) CfA data point for the same entity was followed within a year by a theoretical treatment from Taiwan [45] (apparently the authors were unawares of any measurement of the triplet level lifetime), and within another year by another publication from 
Leningrad [46]; that paper explicitly claimed good "agreement with the experimental results, including the recent ones". When talking to coauthor Vainshtein about this result in the light of later progress in the field, I was told "that this is where experiment was at the time". Evidently the theorists had been satisfied with the outcome of their computations, because it agreed with published experimental data. As an experimentalist I ought to be happy about this trust in experiments, but I am hesitant to rely on computations and experiments that have not been critically evaluated and passed various intrinsic tests, preferably also having been cross-checked against work using other techniques, and so on. Theory claims to be of predictive value; if computations are considered successful because they reproduce experiment (without independent accuracy estimates), then they have no predictive value and should not be considered proper. However, our story continues.

Beginning in 1994, the groups of Alan Hibbert and Charlotte Froese Fischer (and their postdoctoral coworkers), using very different codes, began to place the intercombination transition rate of interest in C III just above $100 \mathrm{~s}^{-1}$, at first with uncertainty estimates of 4\% and 3\% [47-49], respectively, but already stating that their result was incompatible with the aforementioned papers from CfA. In 1996 a new experiment at the Heidelberg heavy-ion storage ring TSR succeeded (published in 1997 [50]). The first case studied with this technique was the very intercombination transition in C III, and the excellent data quality left no options for various ways of evaluation: a single exponential yielded a lifetime (and thus also the transition rate) with an uncertainty of a small fraction of $1 \%$, the most accurate such data point for any multiply charged ion at the time, and in full agreement with the more recent computations. (Curtis [51] lateron has tried to see whether any deviations from an exponential decay law might be recognized in the original data files with their excellent signal-to-background ratio, because that would be of high fundamental interest, but has not found any.) Theory (mostly by the same groups and their associates) since then has dared to specify a theoretical uncertainty of about $1 \%$-again, once one knows the "right" answer, one may feel more confident also about computations that come close. The situation of theory and experiment in Be-like ions then became the topic of a systematic review [27] that included versions of atomic structure packages that are still in use today, and the present manuscript in a way is an offspring of that review, too.

Theory with an uncertainty estimate of $1 \%$, however, does not test an experimental data point of much smaller error bars. Fortunately, Mau Chen, K. T. Cheng and Walter Johnson [52] have mustered the resources of a National Laboratory and applied Relativistic Configuration Interaction (RCI) computations to the problem, with a few hundred thousand configurations. Their computation massively reduced the often astoundingly large discrepancy between results obtained in Babushkin and Coulomb gauges (in nonrelativistic approaches called the length and velocity operator forms), and their error estimate is on the order of half a percent. Their result differs from experiment by slightly more than the combined error bars. In a way, that is a healthy situation. Agreement of experiment and computation is good at a high level in this example, but not perfect. This leaves room for improvement on both sides. However, funding for improved experiments or for even larger computations has not been forthcoming yet. The level of accuracy reached in this atomic lifetime measurement and associated theory carries over to systems which would test QED predictions at the edge of the Standard Model, if only many-body quantum mechanics was developed well enough, as will be discussed later. 


\subsection{General Problems}

The above examples touch on many problems. Within the active time of a given field (fashions come and go also in science) or a given experimental technique, such as beam-foil spectroscopy, there are learning curves of individuals and of the community as well as the development of technical procedures and of equipment. It is important to become aware of laboratories, their attitude, achievements, and possible decline, of people, facilities, funding cycles, alliances and hostilities. This is not limited to experimental work, but also to computations. When computing was still expensive and technically very limited, some shrewd operators (such as Harry Nussbaumer) might find configurations that yielded atomic structure numbers reliably close to reality, from small basis sets that seem ridiculously small in hindsight, while the results obtained by others with similar technical means scattered rather wildly compared to what we know now. The less successful people would not advertise that their means were so limited; it is almost an indicator of self-delusion, if one sees authors praise their own computations without any realistic proof. Nowadays we can expect computations to include convergence tests in which, for example, the number of configurations is systematically varied in order to see whether the result changes significantly. This procedure is expensive in terms of computing time, because it does not increase the number of results, but it is one step of many to estimate the reliability of computations.

Authors with a poor grasp of the concept of reliability seem to prefer to list results with many decimals, most of which are actually meaningless, even as any computer program will reproduce them when starting with the same input. When years ago Pedro Goldman and Gordon Drake had a friendly competition about the accuracy possible in the computation of Bethe logarithms and energy levels in two- and three-electron ions, it was a useful measure to talk about twelfth and thirteenth digits. The two parties challenged each other and tested each other, but always with an eye on the demands of highly accurate experiments. Their computations corroborated experiment at a very high level and then went beyond the experimental data in order to be available when experiment would be improved again. This was excellent work. When nowadays I see manuscripts of computations on core-excited few-electron ions that state ever so many significant digits, I am regularly disappointed, because what matters is not any long number, but usually the difference (term difference) between pairs of them. The really reliable digits disappear in the difference, and the reader is left at a loss when wanting to judge the reliability of the difference.

Of course, this is not a new problem. Dividing one by three leaves you with an infinitely long decimal expression, but of only a single significant digit. Some people write down eight digits from a pocket calculator, or nowadays use double precision computer printouts. This does not enhance the true, inherent accuracy. If an author uses the GRASP code to calculate wavelengths of transitions of ordinary ions in the visible and lists eight significant figures, I know that the author lacks basic physics insight. I have seen tables filling full journal pages with such nonsense, comparing the computations of fine and hyperfine components of lines with poor measurements that yielded (visible) wavelengths with a spectral resolution of maybe a nanometer and a precision that justified no more than 4 significant figures (at best). A journal editor with basic science training should recognize the nonsense, but he/she usually leaves that to a voluntary referee, to whose helpful comments some authors do not concede any value. If rejected by one journal, those authors simply turn to another journal, with the same faulty manuscript. 
I have also seen Indian claims of confirming beam-foil lifetime measurements of three decades earlier, with similar error bars stated as in the sound early papers from American colleagues. (In this paragraph I refer, among others, to a specific author who has published various unscientific (in my eyes also fraudulent) claims in Phys. Rev. A, J. Phys. B, Phys. Scr., Z. Physik, Eur. Phys. J., Nucl. Instrum. Meth.- this is not a problem of one journal or one country or continent.) Alas, in none of the papers submitted by the same cluster of authors it was ever proven that the ion accelerator they had used actually did provide ion beams at an energy necessary to produce the high ion charge states in question. In fact, the lead author relied on a French charge distribution model that had been published by its authors for a much higher energy range. The French authors explicitly limited their validation of the model to a certain range (way above the working range of the accelerator used in the experimental work by "those other authors"). Actually, there are valid charge distribution models for the energy range of interest, and they show that the claims by "those other authors" were untenable (I should say fraudulent). Moreover, "those other authors" for almost all of their work used a solid state X-ray detector that yields basically a single spectral line of the X-ray spectrum where with a proper spectrometer one can see dozens of lines from different charge states (which invalidates any interpretation based on that single line). Thirty years earlier spectroscopic measurements had been run in a physically sound way at various laboratories. One might expect that 30 years later people would build on that knowledge and make progress, but "those other authors" instead have elected to turn to fanciful and modern-sounding interpretations of their single-line $\mathrm{X}$-ray data which are physically unsound and in violation of established knowledge. Yet referees and editors have fallen for the competent-sounding manuscripts from a formerly developing country (countries that boast nuclear weapons do not deserve "helpful refereeing" any longer!) and completely overlooked the rotten base. Unpleasant as it is, one has to consider the existence of science colleagues who are not just doing poor science, but who invent meaning, stylize their own work preposterously, operate at least close to fraud. It does not help the field to let their manuscripts pass into print. Well established international physics journals have violated their own policies against serial publications with such non-physical material and have not shown any inclination to shield themselves against such authors in the future after having been warned. Thus neither the status of a journal nor the peer review system provide any warranty for the soundness of published data. If we know our colleagues, we may form an opinion on their work. Newcomers to the field will need some period of apprenticeship with established work groups to gain personal credibility — whatever atomic structure packages they might use. The same holds for experimenters - they are working in a laboratory set up by somebody else, usually beginning with projects thought up by somebody else, and they need to gain and demonstrate experience on their own. Trust and distrust are human factors, but as discussed above, even when judging dry spectroscopic data, some skepticism about authors and institutions is warranted. Historians have turned to institutions such as the Harvard-Smithsonian Center for Astrophysics and revealed its particularly troubled period in the mid-1950s. In other institutions, too, there must have been ups and downs linked to people and funding, and possibly to scientific quality as well.

Although a commonly trusted large institution such as the National Institute of Standards and Technology (NIST) is seemingly beyond reproach, it would be unlikely not to suffer occasionally from flaws in some or other of its sections. After all, they employ people. Maybe such institutions are too easily seen as a brand name and a guarantor of product quality, instead of their true role as an address 
at which many people work. Still, name recognition may be useful for funding purposes and public relations. However, institutional status and policy can also have drawbacks for science. For example, as part of such an institution, the spectroscopic data base group at NIST feels obliged not to correct even clearly clerical errors in their data base, unless the correction is the result of a fully-fledged research effort of their own with all the in-house refereeing and vetoing prescribed, which is costly and for which the manpower is too scarce. Thus mistakes that have passed by the internal checks and referees into their data compilations can be long-lasting and almost impossible to weed out - as an inadvertent result of their quality-assurance policy. The error rate in the measurements, evaluations, compilations and tabulations by Bill Martin, Victor Kaufman, Jack Sugar, Joseph Reader (to name just a few NIST spectroscopists I had most contact with) and their colleagues is admirably low, but naturally it is not zero.

The sheer size and complexity of any spectroscopic data base such as that built up and maintained at NIST (ASD) have brought about various side effects. No longer are individual entries linked to their sources; the tabulations mix contributions from experiment, derived from a meta-analysis of experimental levels (by the Ritz combination principle), and from computations. A data base aiming at completeness of entries is appreciated, but the data quality is becoming murky. Decades ago a declared goal of several NIST spectroscopy group members was to obtain EUV and soft-X-ray wavelengths with an accuracy of $5 \mathrm{~m} \AA$. By far not all data compiled in the data base have ever come close to that standard. In recent EUV spectroscopy work, a number of NIST ASD spectrum listings have been found to contain much less accurate wavelength data, and there is no simple way anymore to find out what the original error estimate has been. In the very helpful (though dated) Kelly and Palumbo tables [53] the authors state that L. J. Palumbo was responsible for the technical process of porting whatever tabulations into the book format - a formidable task. R. L. Kelly took the blame for all scientific decisions, including the selection of data. That selection, indeed, is where some sources that had stated rather too many digits of the wavelengths were (mis-)judged to be better than others, but actually had been wrong in measurement or line identification. These problems with a group of soft-X-ray publications surfaced when Multi-Reference Møller-Plesset computations were used to interpret high-resolution EUV beam-foil data (see examples and discussion in [54]), and old reference data turned out to be at fault.

By the way, one should also be cautious of new ways of defining error. One group at NIST once introduced a "probable error" different from variance or standard deviation. It made their measurement uncertainties look smaller (when the numbers are expressed in error intervals), but luckily it was not adopted elsewhere. (Although, whenever the community sees the label NIST, people assume that something substantial is being conveyed and try to understand the procedures.) Instead, some eyebrows were raised when the actual NIST X-ray data associated with the new error measures were scrutinized in other laboratories and were found to not support the image of world class reference data. Another nuisance is the claimed 'custom' in various communities to quote one standard deviation (comprising $67 \%$ of the data) or two (95\% confidence interval). On the odd chance that somebody from a different neighborhood should read a paper, it would be best to state what error measure is being used.

Experiment and theory are both needed at a high quality level before one can begin judging the quality of computations by an intercomparison. It is not a suitable measure of reliability if the output of one computation largely agrees with that done by somebody else (an argument frequently encountered in computational manuscripts from non-Western countries). If it does not agree, it is obvious that there 
must be problems, but agreement may just be by chance, or by using a similar algorithm with the same shortcomings (perhaps in a program of a different name). When the Grant code was in its infancy, lifetime predictions showed a significant unreliability. Debugging efforts by members of other groups then revealed that certain sets of integrals had only been estimated (by a prominent theory coauthor) in order to obtain some output (not bad for that). When the numerical problems were solved and the integrals properly solved, the results improved markedly. The successor stages of that development have become mainstays of the atomic structure computation business.

Error estimates sometimes also are parts of political plays. There are groups eager to shave a little off an error bar in order to be seen as better than the competition, and there are others who tend towards more conservative estimates as a matter of principle. In the 1970s, there was a competition for the most accurate atomic lifetime measurements on fast beams of atoms. The group of Horst Jürgen Andrä at Berlin was particularly active and was repeatedly credited with the most important work. In early work on Ba II an uncertainty of $0.1 \%$ was claimed [55], to which no correspondingly precise theory was available for comparison. The laboratory work therefore was re-oriented towards calculable systems such as the alkali atoms where an intermediate experimental result reached $0.26 \%$ (reported at a conference; the final results were later published with error estimates of $0.15 \%$ for $\mathrm{Li} \mathrm{I}$ and $0.18 \%$ for $\mathrm{Na} \mathrm{I} \mathrm{[56]).}$ Some hay was made out of the disagreement of any but the poorest computations with the superb measurements. At the same time the group of Schmoranzer at Kaiserslautern obtained data with a slightly larger error bar [57], but their data were internationally almost overlooked, because the Berlin data seemed superior. Using a different technique, laser excitation of atoms in a vapor target, Jörgen Carlsson at Lund measured lifetimes of the same levels (and applied the same measurement techniques also to hyperfine structure studies), but again at slightly larger uncertainties [58-60]. In the long run, theory was improved until in the end it was accurate enough and then it contradicted the Berlin measurement. After a few years of uncertainty of whom to believe more, a very different technique (using the weak binding between distant $\mathrm{Na}$ atoms [61]) enabled a major improvement. The new data from molecules and from a newer version of the Kaiserslautern fast-beam experiment match recent theory within their $0.2 \%$ uncertainty [62,63], and the continuing work at Kaiserslautern has also indicated a likely minor flaw in the early Berlin work. By the way, both, the original Kaiserslautern and Lund results and the clearly improved new ones, are compatible with theory. So Kaiserslautern and Lund were right from the beginning, but lost out on the advertising side.

The Berlin and Kaiserslautern groups used fast atom beams produced by the neutralization of ion beams in a gas, which is associated with a smaller energy loss than ions experience in an interaction with a thin foil. This energy loss (mean and scatter) is one of the disadvantages of beam-foil spectroscopy, because it affects the knowledge of the ion velocity at observation past the foil. In a Swedish lifetime measurement on the He I 1s3p ${ }^{1} \mathrm{P}_{1}^{\mathrm{o}}$ level [64], the actual ion beam velocity was measured from quantum beats in neutral He atoms, the beat frequency being provided by highly accurate computations of the $n=3$ triplet fine structure intervals. The singlet level lifetime result has been published with an uncertainty of $0.27 \%$ and still is the most accurate traditional beam-foil lifetime measurement result. Most certainly the $0.27 \%$ uncertainty value is the result of a thorough error analysis. However, I have learned [65] that H. J. Andrä, the group leader of Andreas Gaupp, jokingly congratulated Indrek Martinson, the senior scientist in the Swedish group, for having chosen an error bar just a little larger 
than their own (at the time, on a different atomic system and experiment). Knowing Indrek, who in the 1960s had worked alongside Andrä in Stanley Bashkin's newly started beam-foil spectroscopy group at Tucson, I would not exclude the possibility that a politically wise conscious adjustment of error bar size may have occurred rather than sheer happenstance. At least the anecdote reflects the competitive spirit of some actors in the field.

\section{Isoelectronic Sequences}

The recognition of experimental error sources and of error propagation in statistics and data evaluation suggests that we repeat any measurement many times. The finiteness of resources, however, makes us also strive for measurements on other systems. It would be good to correlate the two pools of measurements, for example by studies of ions along isoelectronic sequences. This adds a parameter, the nuclear charge $Z$ or the ion core charge $\zeta$ (the spectrum number), to the pool of data. Hylleraas' series expansions of atomic parameters such as level or binding energies, transition rates, line strengths, polarizabilities, and so on, provides simple systematic interconnects [25,26]. Where series expansions and analytic formulas don't reach, one may turn to simple computations as first approximations that give guidance but eventually need to be replaced by expansive work. Examples of such extremely useful tabulations are the MCDF computations of the energies and rates of transitions in ions isoelectronic with first row atoms (Li through F) by Cheng, Kim, and Desclaux [66] and in Al-, Si-, P-, and Cl-like ions all across the periodic table of elements by K.-N. Huang and his coworkers [67-70] from Taiwan which I have benefited from for decades. (This group uses more up-to-date computational tools nowadays, such as MCRRPA (see [71,72] and much more computing power), but the old tables have not been re-run and re-published.) However, with improvements of spectroscopic tools for beam-foil spectroscopy and with the advent of electron beam ion traps (EBIT), both of which can give access to all charge states of all elements, the Huang tables have reached the limits of their use, because they were computed when computing resources limited such work to rather few configurations and small basis sets. "Modern" data of high spectral resolution need higher quality computational results to compare with. Instead of including examples from those now outdated studies in my examples discussed below, I prefer to expand the scales of the graphs to show what more expansive computations can do nowadays. Similarly, there are series of tabulations of atomic data for such isoelectronic sequences and astrophysical applications (including spectral modeling) contributed, for example, by A. K. Bhatia and G. A. Doschek (Naval Research Laboratory) and various of their colleagues. Those tabulations have had their use and their deserved user community. However, the lowest energy transitions listed in those tabulations are usually the M1 transitions within the ground configuration. Transition rate measurements at heavy ion storage rings and at EBITs have repeatedly shown the tabulations by Bhatia et al. to be off by some $30 \%$ and more for the rates of these E1-forbidden transitions. It is therefore not appropriate any longer to include those outdated computations in a discussion of the state of the art. At the same time I am skeptical when I see that a computation addresses only a single ion species and not at least a few ions along an isoelectronic sequence. A variation of the parameter $Z$ in principle is simple in atomic structure computations, but might reveal quality deficiencies (of computations or measurements) in a comparison 
with further experimental data- $-\mathrm{a}$ chance not to be missed. Moreover, only if a computation reaches a given degree of accuracy before measurement can it boast of significant predictive power.

\subsection{H-Like Ions}

The Dirac equation is often considered as an exact description of the one-electron atom or ion. However, that would require an infinitely heavy nucleus to avoid recoil effects, which apparently cause problems to the formalism. Hence there is no exact description of even the simplest atom. Nevertheless, in the face of a plethora of correction terms and of the series expansions of quantum electrodynamics (QED), the general assumption in the community is that H-like atomic systems can be better calculated than measured. In practice, however, only H I and D I have been measured with extreme accuracy, and for all the ions of the $\mathrm{HI}$ isoelectronic sequence, the tools of study are much blunter.

In order to study higher-order expansion terms of QED, it would be interesting to do accurate measurements on the $\mathrm{H}$-like ion ${ }_{92} \mathrm{U}^{91+}$, because there those terms are relatively larger than the measurement accuracy of the same terms in HI. However, the uranium nucleus is far from spherical, and its imprecisely determined shape causes uncertainties in the computations. In contrast, ${ }_{82} \mathrm{~Pb}^{81+}$ has a doubly magic nucleus with a somewhat smaller nuclear charge and thus smaller QED contributions, but the expected accuracy of experimental tests of the calculated QED terms is expected to be still higher than for ${ }_{92} \mathrm{U}^{91+}$.

There are various computations that address single-electron ions of high- $Z$ elements, especially uranium (for a sample, see [73]), and the differences between the results are much smaller than the precision of direct measurements. A major problem for the experiment is the short lifetime of practically all levels of interest, which by the uncertainty principle is linked to a level width and thus to line broadening. The inter-shell $(\Delta n>0) \mathrm{E} 1$ transition rates scale as $Z^{4}$ as do fine structure splittings and leading QED terms (Lamb shift). The $2 \mathrm{~s}^{2} \mathrm{~S}_{1 / 2}$ level may be long lived in hydrogen $(\tau \approx 1 / 8 \mathrm{~s})$, but its two decay modes, 2E1 and M1 decays, scale as $Z^{8}$ and $Z^{6}$, respectively, resulting in a lifetime prediction of about $9 \mathrm{fs}$ in $\mathrm{U}^{91+}$, with the M1 decay dominating by a factor of 20 over the $2 \mathrm{E} 1$ decay branch. This short lifetime is associated with a considerable line broadening.

An alternative experimental approach uses Li-like ions and builds on theory's success in describing three-electron systems. The experimental idea is simple and exploits the fact that here the $1 \mathrm{~s}^{2} 2 \mathrm{~s}$ level is the ground state and thus not lifetime-broadened. The $2 p$ levels are connected to this ground state by E1 intra-shell decays of comparatively low rate and thus by EUV / soft-X-ray transitions that are not much broadened. Such measurements of $2 \mathrm{~s}-2 \mathrm{p}_{1 / 2,3 / 2}$ transition energies have been done at the Livermore electron beam ion trap [74-76] and in other experiments. In order to estimate the QED contributions in one-electron ions from the three-electron ion measurements, one needs theory as a bridge (with some not quite developed links) telling of the relative sizes of various QED corrections in high- $Z$ ions. In spite of the uncertainties of such a procedure, the result of a measurement on $U^{89+}$ and the extension to $U^{91+}$ by theoretical tools [76] has turned out more accurate as a test of the two-loop Lamb shift contribution to the 2 s level energy in a one-electron ion than any of the attempts at a direct measurement so far. 


\subsection{He-Like Ions}

Singly excited two-electron ions are considered to be accurately calculable [79], both for the level structure and the transition rates among the lower levels. At high $Z$, the longest lived level is $1 \mathrm{~s} 2 \mathrm{p}^{3} \mathrm{P}_{0}^{\mathrm{o}}$ which decays by an intra-shell decay to the $1 \mathrm{~s} 2 \mathrm{~s}{ }^{3} \mathrm{~S}_{1}$ level and by hyperfine mixing to the ground state (see the 4-percent beam-foil lifetime measurement on ${ }_{79}^{197} \mathrm{Au}^{77+}$ [80]). Multi-photon decay rates increase steeply with the nuclear charge $Z$; a quarter century ago Munger and Gould reported a 6-percent lifetime measurement on ${ }_{92}^{238} \mathrm{U}^{90+}$ (even isotope, no hyperfine structure) from Berkeley [81], which recent work at GSI Darmstadt [82] tries to improve on. The easiest detection is by the decay of the $1 \mathrm{~s} 2 \mathrm{~s}{ }^{3} \mathrm{~S}_{1}$ level which is very short-lived, but which is then replenished by the stronger decay branch of the $1 \mathrm{~s} 2 \mathrm{p}{ }^{3} \mathrm{P}_{0}^{\mathrm{o}}$ level. The rate of this decay branch depends on the $2 s-2 p$ energy splitting and can thus be exploited for a determination of the ${ }^{3} \mathrm{P}_{0}^{o}$ level position. In the highest- $Z$ naturally abundant element, uranium, the $2 \mathrm{~s}-2 \mathrm{p}_{3 / 2}$ transition has also been seen by now [83], but such an observation with its considerable uncertainty is no challenge to theory.

At lower $Z$, the longest-lived level is the lowest triplet level, $1 \mathrm{~s} 2 \mathrm{~s}{ }^{3} \mathrm{~S}_{1}$, with its M1 decay (line ' $\mathrm{z}$ ' in plasma lingo). The story of how this line was recognized against prediction in solar X-ray spectra [84], how theory struggled for a while to find a valid isoelectronic scaling of the transition rate, how Gordon Drake developed a description based on a relativistic transition operator and extensive nonrelativistic wave functions [85], how C. D. Lin in his Ph. D. thesis introduced a relativistic correction [86] to Drake's results, and how it then took a quarter of a century before a fully relativistic description including a wave function expansion to all orders became available [79], has been told in various places (among others, $[6,8,10]$ ) where also most of the experimental sources have been cited. Figure 3 shows a plot of the deviation between experimental data and theory along the isoelectronic sequence. Theory predicts a $Z^{10}$ dependence of the transition rate, which corresponds to a variation by 15 orders of magnitude over the range from neutral $\mathrm{He}$ to $\mathrm{Xe}^{52+}$ ions, and experiment largely agrees with theory within the experimental uncertainty. It is crucial to minimize the experimental uncertainty on such fundamental measurements. This demand has been met best in the range from $\mathrm{B}(Z=5)$ to $\mathrm{S}(Z=16)$, with measurements at the TSR heavy-ion storage ring and at the Livermore EBIT [87-92]. Here the typical uncertainty is about $0.5 \%$. This is comparable to the difference between Drake's (corrected) nonrelativistic computations and the relativistic computations by Johnson et al. [79]. Hence experiment is being challenged to reach an even higher accuracy. This experimental progress is most likely to happen in the range of ion trap experiments, where the accuracy already is highest.

The beam-foil technique used at higher nuclear charges [93-96] is not easily trained to achieve uncertainties of only, say, two percent (the measurement on $\mathrm{Nb}(Z=41)$ [95] is a rare exception). Slow ion beams have been applied to the case of $\mathrm{Ar}^{16+}$ which had a history of underestimated systematic errors in the pioneering fast-ion beam measurements at Berkeley. The incorrect lifetime data obtained initially then intrigued various theoreticians and thus yielded a playground for theory development. In the course of that competition a computation seemingly closest to experiment was found to suffer from a sign error, a clear sign that significant errors can be made in either field and need an attentive field of competitors to be spotted and cured. As of now, fast-ion lifetime measurements with serious precautions as well as slow-ion beam measurements based on recoil ions produced in a gas target under fast heavy-ion 
irradiation [97] have yielded $\mathrm{Ar}^{16+} 1 \mathrm{~s} 2 \mathrm{~s}{ }^{3} \mathrm{~S}_{1}$ level lifetimes near $200 \mathrm{~ns}$ that agree with each other and with theory within their experimental error bars of several percent. An improvement may be feasible near $Z=18$ with well controlled slow ion beams, perhaps produced from an electron cyclotron resonance ion source (ECRIS) or an electron beam ion source (EBIS).

Figure 3. Deviation of the measured M1 decay rate from the lowest triplet level in He-like ions ( $Z=3$ through 54) from the results of computations by Johnson, Plante, and Sapirstein (JPS) [79]. The experimental data points result from work at the Heidelberg heavy-ion storage ring TSR, the Livermore electron beam ion traps (EBIT), a recoil ion beam experiment at GSI Darmstadt, and from beam-foil measurements in various places (Berkeley, Argonne, Darmstadt). Some older results that have been technically superseded are not shown.

He sequence $2{ }^{3} \mathrm{~S}_{1}$ lifetime

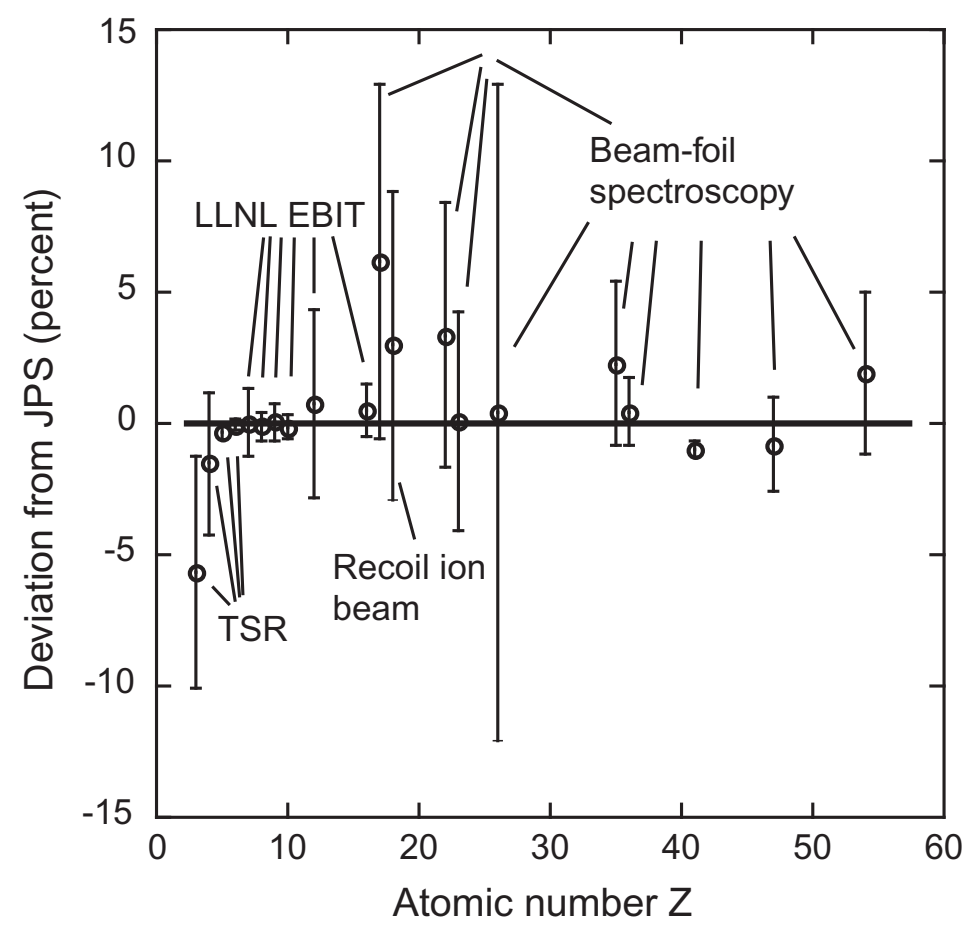

At low $Z$, the $1 \mathrm{~s} 2 \mathrm{~s}{ }^{3} \mathrm{~S}_{1}$ level lifetime in neutral He is a true challenge because of its magnitude on the order of one hour (see the pioneering measurement by Moos and Woodworth [98]). There is a $\mathrm{Li}^{+}$ data point with a $1 \sigma$-uncertainty of $10 \%$ from a measurement at the Heidelberg heavy-ion storage ring TSR that probed the $1 \mathrm{~s} 2 \mathrm{~s}{ }^{3} \mathrm{~S}_{1}$ level by an electron beam and dielectronic recombination. A significant contribution to the error budget is the shortening of the apparent lifetime by ion losses from the ion cloud circulating in the storage ring. In this case the ion loss rate was close in magnitude to the radiative decay rate. A later attempt at TSR has aimed at optically probing (by further laser excitation) the ${ }^{3} \mathrm{~S}_{1}$ level population of $\mathrm{Li}^{+}$ions. However, the laser-ion interaction appeared to introduce new problems, and the experiment has remained unfinished [99]. The TSR storage ring has since been closed down and will be moved to the ISOLDE facility at CERN. A new lifetime measurement at a storage ring with longer storage times, perhaps achieved by an even better vacuum enabled by a cryogenic vacuum 
vessel, seems a worthwhile enterprise in order to test theory in a near-neutral atomic system, which is particularly challenging.

\subsection{Li-Like Ions}

\subsubsection{Levels}

Although a bit dated by now, the 1991 publication by Yong-Ki Kim and colleagues on the resonance levels in $\mathrm{Li}^{-}, \mathrm{Na}-$, and $\mathrm{Cu}-$ like ions [100] still is a most useful benchmark paper, showing excellent agreement of these accurate computations with many-but not all—experimental data. Their computations combine relativistic many-body perturbation theory, Dirack-Fock computations and approximate "screened" QED corrections. Blundell [101] has treated QED in ions of the same sequences in a different way, and experiment indicates that some of his results are superior at very high $Z[102,103]$. However, his computations address only a selection of elements, and thus a direct comparison with experimental results is possible only in a few cases. This problem aggravates with some of the most detailed computations which often deal only with a single element, or with a few elements with isotopes that offer a chance at studying hyperfine effects (which scale with $Z^{3}$ ). There is a cottage industry of computations of higher-order QED contributions and nuclear magnetic effects to the level energies of Li-like (and H- and B-like) ions of a few promising isotopes. However, this view to the detail appears to blind out the larger picture and isoelectronic trends. If an isotope has not yet been studied for hyperfine effects experimentally, it is much less likely to find theoretical treatment. Of course, it is uneconomic to calculate all isotopes in detail, but it might be useful to identify isotopes that from a theoretical perspective (perhaps taking elemental abundances into account in the selection) appear to be more promising than others.

In the mid- $Z$ range, data have been pursued at tokamaks [104] and by beam-foil spectroscopy in a Giessen-led collaboration working at GSI Darmstadt [105-109], corroborated and almost matched in accuracy by EBIT work at Livermore [102]. EBITs have the advantage of a practically stationary light source where beam-foil spectroscopy suffers from having to correctly account for the Doppler shift of light emitted by a beam of fast ions. The mid- $Z$ range is of interest, because relativistic effects are notable, but not yet dominant. (Appropriate theory has been discussed in the aforementioned references.) With increasing ion atomic number, both, relativistic effects and QED contributions, increase and require fully relativistic computations, not just nonrelativistic computations with relativistic corrections. Nuclear physics effects also change with the atomic number. Very heavy nuclei are usually nonspherical, and the correction of nuclear size effects in QED that are related to actual nuclear structure is difficult, because the nuclear parameters of the heaviest naturally abundant isotopes are not known well enough. Hence QED effects are largest in uranium $(Z=92)$, as has been confirmed by measurement and theory for various highly charged ions [74-76], but for a long time the nuclear structure uncertainties posed a limit to accurate computations. Because of this, more accurate tests of QED contributions than with $\mathrm{U}$ may be doable with $\mathrm{Pb}(Z=82)$, although the absolute magnitude of the QED effects is smaller. Recent development in theory begins to shift this notion, and with improved modeling of the nuclear shape [77,78] now higher-order QED effects may be the main source of uncertainty. 
Because the weight of various entities and processes changes in atomic systems and in computations along isoelectronic sequences, one should not expect that a single computational approach easily matches all data. There are wide gaps in the upper half of the $Z$ range of elements, and that implies that the mutual consistency of the experimental data has not yet been assured by sheer statistics. Experimental problems with the ions of any given element possibly still play an unrecognized role which may only be found out when the data points are closing up to each other. If such problems have been overcome, the differences between measured and calculated data points (level energies, wavelengths, lifetimes, etc.) should follow smooth trends that can be interpreted for clues to missing contributions to atomic structure computations. As will be demonstrated in the subsequent sections, "we are not there yet". As a general observation, wide-range computations may be in excellent proximity to low- $Z$ data and yet be imperfect elsewhere. Atomic structure effects may appear as missing overall terms or as a change of balance of configuration interaction, relativistic effects, and QED contributions.

In comparisons of measured and calculated values, one would like to show the difference from "truth" which indicates how much further one has to go. Since truth is no physical entity and the correct numbers are not known, one could compare calculated values to measured ones (which bear an error bar), or measurements and computations to one of the latter (which ought to carry an error estimate). In the former presentation, the scatter of measured data is reflected in an opposite scatter of all other data entries. Because computations tend to yield a smooth trend and for the fact that computations can be done for each atomic number while measurements often cannot, the most commonly chosen option is the reference to one computation. This choice does not preclude the possibility of errors and inconsistencies in the trend of the computed numbers.

\subsubsection{Lifetimes}

Over the years, the $2 \mathrm{p}_{1 / 2}$ level lifetime in Li-like ions has been measured from $Z=3$ up to $Z=92$, and the $2 \mathrm{p}_{3 / 2}$ level lifetime from $Z=4$ up to $Z=36$ ( $\mathrm{Li}$ I was not included here because of quantum beat problems). This situation reflects the absence (largely) of relativistic effects in the $2 \mathrm{p}_{1 / 2}$ wave function and the proportionality of the level energy to $Z$, whereas the fine structure splitting, a relativistic effect (proportional to $Z^{4}$ ) massively modifies the $2 \mathrm{p}_{3 / 2}$ level energy. The steeply increased transition energy shortens the $2 \mathrm{p}_{3 / 2}$ level lifetime to a few picoseconds in $\mathrm{Kr}^{33+}$ [20], whereas the $2 \mathrm{p}_{1 / 2}$ level lifetime amounts to much more than that even in $\mathrm{U}^{89+}$ ions, namely about 62 ps [110]. These lifetimes in high- $Z$ Li-like ions are in good agreement with the venerable ab initio MCDF computations by Cheng, Kim and Desclaux [66]. Theodosiou, Curtis and El Mekki [111] have tried a combination of simpler computations (the numerical Coulomb approximation, which actually predates the MCDF approach by many years) in combination with corrections for experimental level data. Their parametrization effort yields rather similar level lifetimes for practical applications. However, their procedure is not a show of quality of theoretical achievement, but rather a show of what lengths one has to go for in the absence of sufficiently good ab initio predictions. The complete lifetime data set has been analyzed again elsewhere (see Figure 1 of [21], where also the references to the experimental data are given). Theoretical interest in the Li sequence has not waned, and only recently a stretch of the sequence has been recalculated for levels, lifetimes and collision strengths [112,113]. By the way, at low $Z$ there are two early beam-foil results for the $2 \mathrm{p}_{3 / 2}$ level lifetime in $\mathrm{Be}^{+}$, neither of which agrees with the mean trend-but the 
weighted mean of the data does, which suggests that at the time the experimental uncertainties may have been underestimated.

Another caveat for the search for 'best' data: The latest publication is not necessarily the most advanced one. For years, L. J. Curtis maintained a web page of his publications that also offered access to a manuscript by Kukla et al. in Phys. Rev. A style formatting. The manuscript reported on beam-foil observations of Li-like ions of $\mathrm{Kr}$, but it never appeared in the journal. At one stage the manuscript was cited by colleagues who had measured Li-like ions of Ni [114] and seemed to believe that the Kukla work was much younger than a 1995 publication on Li-like Kr [20]. However, I had met Kris Kukla on another project at Argonne when he was active in the field. Thus I had an inkling of the true time frame, and I knew of such (partial) data from Argonne mentioned in a 1999 conference paper [115]. From Curtis I learned that the plan had been to write a conclusive Kr paper on the basis of Kukla's thesis work, and the version offered on the web had (largely) been an unedited chapter of that thesis. I urged to see this material finally brushed up into a proper publication [116], thus adding another data point to the collection, in order to accompany my own earlier lifetime data for $\mathrm{Kr}^{33+}$, albeit from a more limited data evaluation. Still, a second data point (actually, in this case a third) is a good thing for a data base as it indicates the reproducibility.

It is not likely that much more lifetime work will be done on the $2 \mathrm{p}_{3 / 2}$ level between $\mathrm{Li}$ and $\mathrm{Kr}$, because the data collected so far seem consistent. However, higher accuracy, possibly better than $1 \%$, would be valuable. Selective excitation of trapped ions by VUV or EUV laser light and electronic timing might do for the first few ionization stages. An alternative for more highly ionized species, and with the potential of reaching high accuracy, might employ X-ray laser excitation starting from ions prepared in an EBIT [117]. The lifetime of the other fine structure level, $2 \mathrm{p}_{1 / 2}$, could thus be studied in all high- $Z$ ions. For this level even the proven technique of beam-foil spectroscopy would do in principle (if practically limited to an accuracy rather above than below 1\%), but no accelerator laboratory presently seems interested in such a project.

While the spectra of Li-like ions are dominated by the excitation of the single valence electron outside a closed shell, this is by no means the only option of interest. Double and triple excitation are feasible and cause satellite lines to the diagram lines of $\mathrm{H}$ - and He-like resonance line series in the X-ray spectra of many light sources (plus two-electron one-photon satellites). Since a long time the satellites have been exploited as a diagnostic of temperature and density in astrophysical plasmas and in fusion-oriented plasma research. Beam-foil spectra show a profusion of such satellite lines because of the high electron density (and thus a high collision frequency) in the excitation volume (the foil) [118-120]. Most multiply excited levels decay rapidly, often by autoionization, a process which in low charge state ions has time scales on the order of ten femtoseconds. However, symmetries sometimes hinder autoionization and/or $\mathrm{X}$-ray decays, and a triply excited level of neutral Li has been observed and its picosecond level lifetime measured [121-123], both in good agreement with computations. The wavelength measurement on the decay of the triply excited system incidentally represents one of the most accurate such measurements of beam-foil data in the EUV. For many less special levels the calculated results have been less fitting the anyway not very accurate experimental results $[124,125]$. This experience cautions against any general claims that three-electron systems are reliably calculated these days. What can be calculated 
well are singly excited three-electron systems in which the single valence electron outside a closed-shell core dominates.

Years ago a triply excited few-electron ion seemed rather exotic and far from any use. Nowadays synchrotrons, EUV- and X-ray lasers have become such brilliant light sources that with their intense radiation fields they can produce multiply excited ions from neutral matter. However, so many multiply excited levels can be reached (though not necessarily resolved) in the same energy pulse that in the face of such complex spectra so far no highly accurate numbers are needed for modeling and understanding the radiative properties of specific systems or their individual levels.

\subsection{Be-Like Ions}

Four-electron ions (partly discussed in the introduction), with two electrons in a closed $n=1$ shell and two valence electrons, add a lot of complexity to that of three-electron ions. Even within the lowest shell ( $n=2$ ) we now have not only electric dipole (E1) transitions (such as the resonance transition), but also a spin-changing E1 (intercombination) transition, M1 transitions between the $2 \mathrm{~s} 2 \mathrm{p}$ triplet levels, an E2 transition between the $J=0$ and $J=2$ triplet levels, $2 \mathrm{p}^{2}$ displaced levels, multiplet mixing, magnetic interactions, and so on, and the various interactions scale differently with $Z$. An exact atomic structure computation would return correct results on all atomic parameters. Existing programs do not achieve that goal. They usually employ approximations and address certain aspects, and we should not be surprised that computations that yield numbers in agreement with experiment for one atomic property do not necessarily serve equally well for the others.

Ulyana and Marianna Safronova, Walter Johnson, and their colleagues have studied various details of Be-like ions along the isoelectronic sequence by RMBPT computations [126-128]. Their plethora of results would not fit into any print journal, which is why numerical data often have been tabulated for a few ions only, and trends (especially useful for warning of cross-overs among levels and changes of their labeling) have been demonstrated graphically. It comes as no surprise that a schematic selection of ions (such as every fifth or tenth element) is not likely to provide data on all elements of interest to experimenters, and single-column wide graphics covering data of the whole table of elements that vary by orders of magnitude are not suitable to extract accurate values. The constraints may be imposed by economic limitations, but they also limit the feasibility of comparisons between experiment and computation. For low-lying levels in Be-like ions, among the more recent computations (two decades younger than the venerable MCDF computation by Cheng, Kim and Desclaux [66]) the most dense listing appears to be one of the papers named above [126], making it the most suitable reference for comparisons. Figure 4 shows that the experimental data points are internally consistent up to $Z=36$, but are very few and less precise above. As to the quality of the computations, the agreement with experiment is best near $Z=20$ and clearly poorer below and above this range. The three experimental results for high $Z$ elements originate from different light sources, for Mo $(Z=42)$ a tokamak plasma [104], and for Xe $(Z=54)$ a beam-foil experiment [108] and an electron beam ion trap [102], and they seem to indicate a different trend than the low- $Z$ data do. More experimental data are needed, and other types of computations would be welcome, too. There are, for example, the RCI computations by Chen and Cheng [129] that, however, treat even fewer ions than the Safronovas and Johnson. At low 
$Z$, these computations are practically coincident with the experimental data (in contrast to Safronova and Johnson); beyond $Z=30$ they deviate slightly from the perceived trend of deviations between experiment and MBPT results, and at high $Z$ the deviation between RCI and MBPT changes sign. Hence there are significant differences between the results obtained with highly developed atomic structure codes, and in this case RCI seems to do better. (Preliminary Livermore EBIT data for $\mathrm{Pb}(Z=82)$ fall close to the RCI predictions.)

Figure 4. Measurements (full black symbols) and Relativistic Configuration Interaction (RCI) computational results (open blue squares and broken line [129]) of the $2 \mathrm{~s} 2 \mathrm{p}{ }^{3} \mathrm{P}_{1}$ level in Be-like ions and their deviation from Many-Body Perturbation Theory (MBPT) computations by Safronova et al. [126]. Note that at low $Z$, the computation by Chen and Cheng [129] is close to experiment, while the other one is not, and vice versa at higher $Z$.

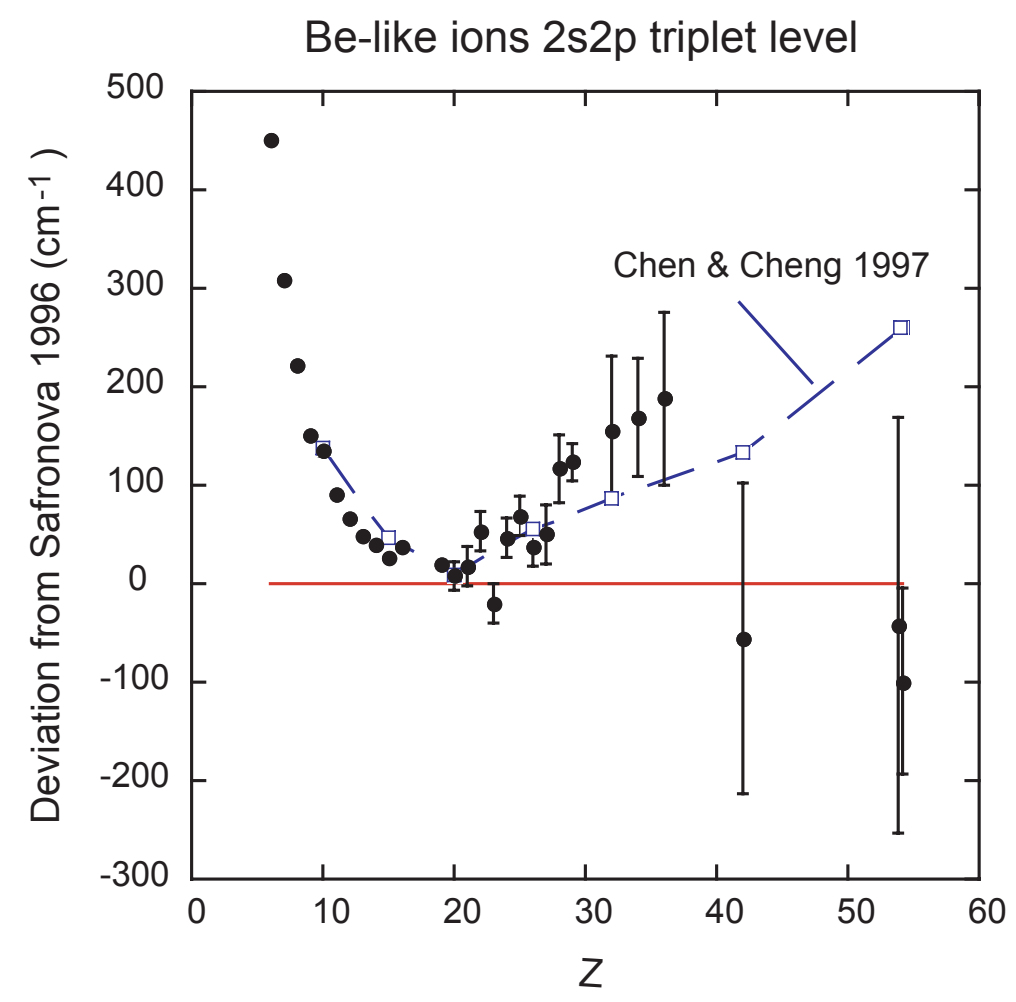

\subsection{1. $2 \mathrm{~s} 2 \mathrm{p}$ Level Lifetimes}

For comparisons with experiment it is helpful to find predictions for all elements. Träbert and Curtis [21] have listed much of the experimental lifetime work on ions in this isoelectronic sequence as well as compared the results to simple scaling rules in order to avoid the possible errors of new computations. Because the references are available there, the present discussion focuses on the state of the art computations.

Per Jönsson and Charlotte Froese Fischer have calculated the $2 \mathrm{~s}^{2}{ }^{1} \mathrm{~S}_{0}-2 \mathrm{~s} 2 \mathrm{p}{ }^{1} \mathrm{P}_{1}^{o}$ resonance transition rates for many values of $Z$ [27]. However, the computations converge better for a more dominant central Coulomb field, that is, for higher $Z$. Computations for low- $Z$ ions thus require more elaborate wave functions, with a larger number of configurations, for the same estimated level of accuracy. Considering the much increased computer run time requirement with larger sets of wave functions, the computations 
then are not formulated identically for different ions. In general, the drop in computing costs is successively permitting the use of larger basis sets for the benefit of computational accuracy. However, not all computations are being updated when the occasion arises, and computations of different quality may be contributing to the same data collection.

Froese Fischer and Tachiev have a web site (presently hosted at NIST) that lists their own MCHF/MCDF/MCDHF results [28]. For Be-like ions, the tables cite MCHF computations of 1999 (for $Z=4-12$ ) and MCDHF computations of 2004 ( $Z=9-30)$ and 2009 ( $Z=10-30)$. No single set of computations covers all elements. What then can one use as a reference to compare experimental data and the results of other computations with? Irrespective of claims at some time that the computational techniques are so good that the resonance transition rate in Be-like ions may well be known (from computations) with an accuracy on the order of $10^{-4}$, the differences among the corresponding tables on this web page amount to several percent. One has a choice if several computations are listed for a given ion, but at low $Z$ there are results only from the oldest computations by the team. This does not matter much for the singlet level lifetime, for which experiment has an uncertainty of about $3 \%$. This can be seen in Figure 1 where the experimental data are referred to the Froese Fischer and Tachiev data collection; the figure reveals that a semiempirical analysis of the same data by Curtis and Ellis [29] describes the isoelectronic sequence equally well, but slightly differently at low $Z$. There is an amusing detail for neutral Be (already discussed above): One analysis favors one of the recent experimental results, and the other favors the other.

For the $2 \mathrm{~s} 2 \mathrm{p}{ }^{3} \mathrm{P}_{1}^{\mathrm{o}}$ triplet level lifetime (see Figure 5) the inconsistency of computational results presented on the same web page [28] is notable. The MCDF/MCDHF computations accessible there have used different numbers of configurations along the isoelectronic sequence, and they did not include $\mathrm{Xe}(Z=54)$, the highest $Z$ data point long since established by experiment [130]. (The result obtained by Cheng, Kim and Desclaux [66] for $Z=54$ was used as an unmarked stand-in in Figure 5, although the isoelectronic trend of those computations is very different and approaches the newer results only in this range.) At low $Z$, results of computations of different vintages are available on the Froese Fischer and Tachiev web page: MCHF for $Z=5-12$, MCDHF of 2004 for $Z=9$ to 30, but leaving out $Z=10$, and MCDHF of 2009 for $Z=10$ to 30, but leaving out $Z=14$. When comparing data outside the range of a single computation, one finds that the MCHF and MCDHF computations by the same team of theorists differ by $7 \%$ (at low $Z$ ); the two sets of MCDHF computations differ from each other by $3 \%$ at $Z=11$ and by about $1.5 \%$ at $Z=30$. Needless to say, it is difficult to trust a computation to, say, $1 \%$, if ever so often new runs by a similar suite of programs result in output that differs by a multiple of the claimed uncertainty from previous results. At low $Z$, the computations by Brage, Fleming, and Hutton [131] (which, alas, do not treat all elements) agree best with the rather accurate atomic lifetime data from heavy-ion storage ring experiments. For $\mathrm{Ne}(Z=10)$ and above, most of the experimental data are rather poor and cannot discriminate between the more modern computations. This calls for further meaningful experiments, for example on $\mathrm{Ne}$, since the present "experimental lifetime" literature entry for $\mathrm{Ne}$ is an estimate from plasma observations which is based on theory plus a $50 \%$ error estimate, and that does not really imply a meaningful measurement. 
Figure 5. Lifetime of the $2 \mathrm{~s} 2 \mathrm{p}^{3} \mathrm{P}_{1}^{\mathrm{o}}$ level in Be-like ions (intercombination decay) displayed as the relative deviation from the computational web resources (MCHF/MCDHF collection) made available by Froese Fischer and Tachiev [28]. A problem lies in the only partial coverage by any given computation, and therefore one cannot refer all of the available measurements and computations to a single reference data set. Because of this, the trends shown all suffer a step function (by $7 \%$ ) above $\mathrm{Ne}(Z=10)$, because this is where one of the references ends and the comparison changes to another one. The only continuous reference data set would have been the one derived by a semiempirical averaging procedure applied by Curtis and Ellis [29] (broken red line) to computations and measurements, which thus represents a secondary trend. The broken blue line refers to computations by Jönsson and Froese Fischer [27], the short-dash broken black line connects the computational results reported by Brage et al. [131] (open circles, the study has to be lauded for point-by-point estimates of uncertainty). Not all of the experimental data (filled circles) are shown, because for several of them the size of the error bars exceeds the plot boundaries.

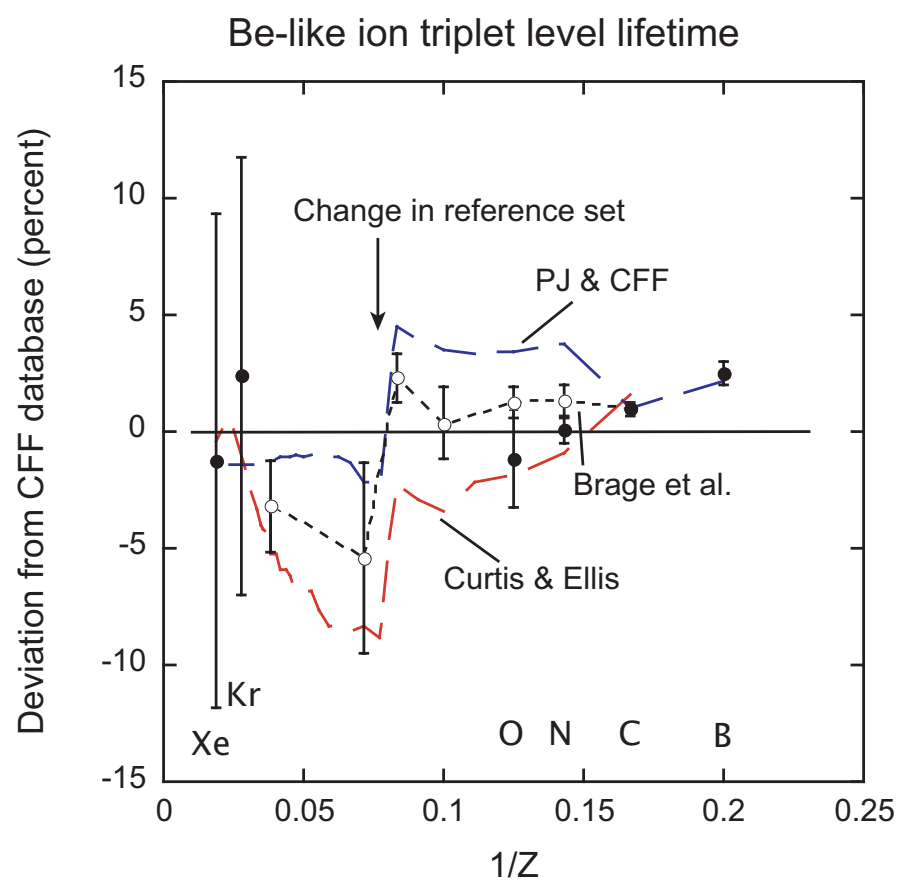

\subsubsection{Displaced Level Lifetimes}

When trying to measure the lifetime of the $2 \mathrm{~s} 2 \mathrm{p}{ }^{1} \mathrm{P}_{1}^{\mathrm{o}}$ level in Be-like ions, especially in beam-foil spectroscopy, one has to also obtain information on the lifetimes of the strongest cascades that replenish this level, which originate from the $2 \mathrm{p}^{2}{ }^{1} \mathrm{~S}_{0}$ and $2 \mathrm{p}^{2}{ }^{1} \mathrm{D}_{2}$ levels which in turn are replenished by other levels. Also, there is the prominent $2 \mathrm{~s} 2 \mathrm{p}{ }^{3} \mathrm{P}^{\mathrm{o}}-2 \mathrm{p}^{2}{ }^{3} \mathrm{P}$ line multiplet. (Similar transitions connecting to displaced levels are numerous in the further isoelectronic sequences.) A rather reliable resource on these transitions has been provided by Brian Fawcett [132] who has catalogued wavelengths and transition rates for L-shell ions up to $Z=28$ from a combination of observations and scaled Cowan code (and variants) computations. Within the typical $10 \%$ uncertainty of beam-foil spectroscopy, the most widely employed technique for lifetime measurements on such systems, the Fawcett tabulations have been found 
to be pretty reliable. This compilation is very valuable for the practitioner; I am not aware of any $a b$ initio computation with a similarly wide scope and accuracy of the results. Some specific cases will be discussed below.

Fawcett's computations are basically nonrelativistic (with relativistic corrections). However, there are effects of multiplet mixing that are much more pronounced than fine structure splittings. For example, with increasing nuclear charge $Z$, there increases the probability of a spin-changing decay branch of the $2 p^{2}{ }^{1} \mathrm{D}_{2}$ level to the $2 \mathrm{~s} 2 \mathrm{p}{ }^{3} \mathrm{P}_{1,2}^{\mathrm{o}}$ levels. (A corresponding case, the $3 \mathrm{p}^{2}{ }^{1} \mathrm{D}_{2}$ level decay, also with in-system and intercombination decay channels, occurs in the $\mathrm{Mg}$ isoelectronic sequence $[32,133]$.) One might expect that any atomic structure code operating in intermediate coupling would include this decay branch automatically, but the scarcity of literature data and the scatter of the results of the few available computations tell a different story.

\subsubsection{Hyperfine-Induced Decays}

Just as the $1 \mathrm{~s} 2 \mathrm{p}^{3} \mathrm{P}_{0}^{\mathrm{o}}$ levels in He-like ions with a non-zero nuclear spin can be made to decay by hyperfine interaction (which mixes the level with a hyperfine level of the nearby $1 \mathrm{~s} 2 \mathrm{p}{ }^{3} \mathrm{P}_{1}^{\mathrm{o}}$ level), the $2 \mathrm{~s} 2 \mathrm{p}{ }^{3} \mathrm{P}_{0}^{\mathrm{o}}$ levels in Be-like ions can be made to decay by hyperfine mixing with a hyperfine level of the nearby $2 \mathrm{~s} 2 \mathrm{p}{ }^{3} \mathrm{P}_{1}^{\mathrm{o}}$ level. The intercombination transition rate in Be-like ions is very much lower than that in He-like ions, and so must be the hyperfine-induced decay rate, requiring a combination of highly charged ions and ion trapping techniques. Hence this decay rate has been measured in only a few ions. Theoretical discussions of the situation have been around for several decades, but have recently been modified considerably. Cheng, Chen and Johnson [134] have recently looked into this problem and explained several computational approaches, which is very helpful for the community. The newer computations largely agree with each other, but the "agreement" with experiment is a few percent in one ion and about $20 \%$ in another (which represents a massive improvement on previous treatments!). This situation is not yet satisfactory, as has been discussed elsewhere [135], and more measurements are sorely needed. It is too early to make sensible statements on the quality of computations in this case.

\subsection{B-Like Ions}

With more electrons in the atomic system, the degree of complexity rises. In addition to inter-shell and intra-shell transitions without or with a change of spin, regular and displaced levels (already present in Be-like ions) there now are E1-forbidden transitions within the ground term. Such (E1-) "forbidden" transitions are of wide-spread importance in plasma diagnostics, because their intensity is sensitive to electron density in a much lower range than intercombination (spin-changing) or E1-allowed transitions. In low-charge ions, they serve to understand the extremely dilute planetary nebulae; in moderately charged ions of iron group elements they help to investigate solar and other stellar coronae, and in highly charged heavy ions, where their rate (climbing steeply with $Z$ ) is no longer low in comparison to E1 transitions, they signal the presence of certain charge states and thus serve as temperature diagnostics. Decades ago, Kaufman and Sugar [136] have compiled a hugely useful collection of wavelength and transition rate data on such E1-forbidden transitions up to $Z=42$ (Mo), which in turn has also contributed notably to the NIST data base, combining experimental data with 
(Cowan code) computations of their own. Isoelectronic lifetime studies by experiment, backed-up by modern computations, however, have revealed a fair number of inconsistencies in those tables. Some of those lifetime measurements (mostly done at a heavy-ion storage ring, some at electrostatic ion traps or at EBITs) are cited in the following sections.

Inspired by Bengt Edlén's early systematizations of level data, a number of computations have produced results for ions in the first third of the periodic table, but far from covering all elements in this range (see, for example, [137-145] and further references therein). This patchy coverage renders intercomparisons for extended sections of the isoelectronic sequence difficult or impossible. On the upside, many computations include those of transition rates in addition to levels. Hence measured lifetimes of displaced levels (for Si and P see [146,147], the latest such study may have been the one by Tordoir et al. on $\mathrm{Na}$ [148]) could be and have been compared to theory, whether semiempirically flavored [132] or calculated ab initio [142]. One way out of the page limit of printed journals are electronic depositories. Apparently the authors of the latter work were forced by referee suggestion (or by the journal) to put some of their tables (for three elements) into an electronic depository with the publishers instead which, because of mislabeling, seemed inaccessible 14 years later. (Thanks to A. E. Kramida for pointing out the Erratum with the correct file path!). For anybody interested in the data it is difficult to understand why thus two pages were saved from a print journal, one of which was lost again because of the need for an Erratum.

In B-like ions (and correspondingly in Al-like ions, etc.), several fine structure levels of the displaced configuration $2 \mathrm{~s} 2 \mathrm{p}^{2}$ cross each other along the isoelectronic sequence. The level mixing affects the relative line intensities of $2 \mathrm{~s}-2 \mathrm{p}$ transitions. This effect has been systematically studied in beam-foil spectra $[149,150]$; the observations qualitatively confirm various computational predictions.

A key role in B-like ions is played by the fine structure splitting of the ground term, $1 s^{2} 2 s^{2} 2 p$ ${ }^{2} \mathrm{P}^{\mathrm{o}}$ (Figure 6). The MBPT computations by Safronova et al. [138] approximate the experimental level splitting well up to about $Z=36$, and then they gradually, but distinctly, deviate (see [102]). Experimental data at high $Z$ are sparse [102]. For spectroscopy there also is a complication: the $2 \mathrm{~s}^{2} 2 \mathrm{p}$ ${ }^{2} \mathrm{P}^{\mathrm{o}}$ ground state fine structure interval increases with $Z^{4}$, and so does the fine structure splitting of the lowest $2 \mathrm{~s} 2 \mathrm{p}^{2}$ term, ${ }^{4} \mathrm{P}$. The fine structure splittings grow faster than the $2 \mathrm{~s}-2 \mathrm{p}$ electrostatic level splitting (which increases approximately linearly with $Z$ ), and therefore at some value of $Z$ the lowest quartet level drops below the upper level of the ground term. In addition to the M1 decay that connects the upper with the lower fine structure level of the ground term, there then is the alternate decay path via two intercombination transitions and the lowest quartet level. Incidentally, in Xe the energies of the two intercombination transitions are close to each other and can be obtained from the same spectrum (see [102]). Having to combine results from two spectral lines increases the overall uncertainty, but the measurement takes advantage of a less crowded portion of the EUV spectrum-a measurement on the single-photon decay branch is not likely to provide a more accurate result with present equipment. 
Figure 6. The $2 \mathrm{~s}^{2} 2 \mathrm{p}{ }^{2} \mathrm{P}^{\mathrm{o}} J=1 / 2-J=3 / 2$ ground state fine structure splitting in B-like ions, shown as the deviation from the computations by Safronova et al. [138]. Up to about $Z=30$, experiment (filled symbols) and other computations (open symbols) stay close to the trend predicted by Safronova et al., but then they diverge. Unfortunately, in the latter range the accurate computations are extremely sparse. The EBIT data point for Xe $(Z=54)$ represents the sum of two transition energies, because the lowest quartet level drops below the upper fine structure level of the doublet ground term and thus opens an alternate (two-step) decay path to ground that is more easily observed and resolved spectroscopically than the single-step decay branch.

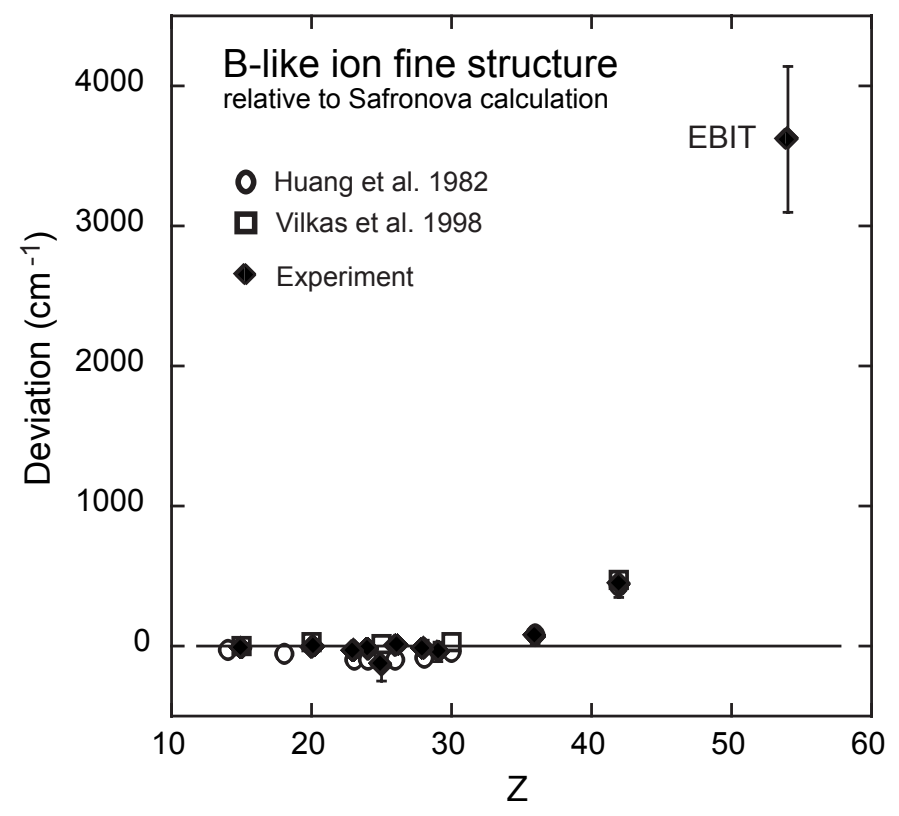

\subsubsection{E1-Forbidden Transition Rates}

The M1 transition rate in the ground term is of some interest in collisional-radiative spectral modeling. Given the vagaries of determining line intensities and line ratios, an accuracy on the order of one or a few percent seems sufficient in practice. Such an accuracy can be expected from a combination of transition energy and line strength. In a first approximation (and a single-configuration picture), the line strength is an exact factor readily determined from Racah algebra. Rather many publications have produced M1 rates for this and related electric-dipole forbidden transitions in a wide variety of ions of astrophysical interest, and the published results have usually agreed to within a few percent with each other and with the above estimate based on transition energy and line strength. However, the actual situation is much more complex. Many people have tried to calculate the fine structure interval (the transition energy), and most computations have fallen short of an accurate result. If one then honestly combines the poor calculated transition energy value with the easily obtained line strength approximation, the transition rate deviates from expectation (see Figures 1 and 2 in [151]). It is deceiving to see most computational papers reporting "good" results, because when the computed energies are poor, a standard escape is the so-called "semi-empirical" correction (recently and more correctly called "rescaling to experimental wavelengths") by which one's own poor energy intervals are replaced by the experimental ones (from well-resolved spectroscopic observations), and then the combination of known energy and geometric 
factors automatically yields results close to the pack. These results are then (probably) close to the 'true' ones and provide a useful guide for the experimenter, but they have not been consistently obtained from atomic structure theory.

Progress has been made in computations thanks to cheap computing power, and nowadays more and more computations are arriving at results closer to the experimentally known fine structure intervals. If the transition energies of these E1-forbidden transitions can be calculated, the second factor, the line strength, becomes of interest in more detail, because it reflects the multi-configuration composition of the wave functions. Fortunately, ion trapping techniques (using EBITs or heavy-ion storage rings, including a cross-check for consistency [152], see the original work cited in the reviews [6-12]) have addressed the millisecond lifetime range and nowadays can measure the transitions rates of many of those E1-forbidden transitions in the ground configurations of multiply charged heavy ions, and they often reach accuracies of better than one percent. One percent-surely computations can do better than that? Skipping all details, the lifetime predictions for levels in the ground configurations often differ by several percent even among more recent computations (Figure 7).

Figure 7. Transition rate of the $2 \mathrm{~s}^{2} 2 \mathrm{p}{ }^{2} \mathrm{P}^{\mathrm{o}} J=1 / 2-J=3 / 2$ ground state fine structure transition in B-like ions, depicted as the deviation from the predictions by Rynkun et al. [144]. Measurements have been done at an electrostatic ion trap [153], electron beam ion traps [154-157], and at a heavy-ion storage ring [158]. The EBIT measurements have eventually reached high accuracy, as is seen in the data point for Ar that has an uncertainty smaller than the data marker. Three representatives of recent computations are shown (identified by the lead authors), and the best experiment [156,157] agrees with only one of them, the computations by Rynkun et al. [144] (full line). It would be good to know whether this computation has taken the anomalous magnetic moment of the electron (EAMM) contribution to the M1 transition moment into account, an effect that amounts to $0.45 \%$ and thus to much more than the experimental uncertainty (smaller than the symbol size), but I have not seen it being mentioned.

B-like ions gs fs $\mathrm{M} 1 / \mathrm{E} 2$ transition rate

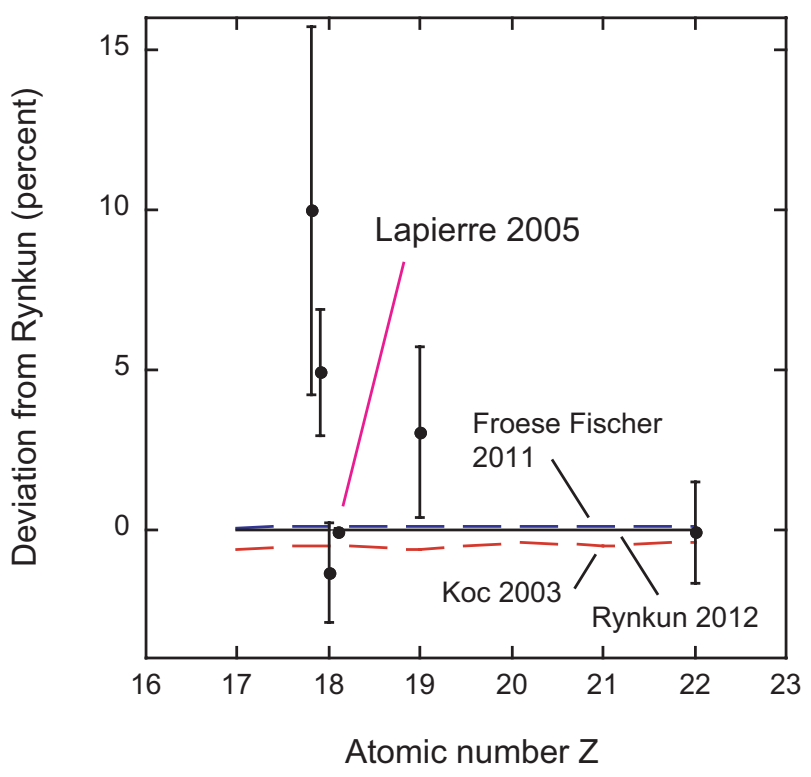


Is it worth worrying about this scatter? Does it matter-since it hardly did matter for decades? Several measurements of E1-forbidden transition rates have reached an accuracy of better than $0.5 \%$, which implies a significant challenge and requires a qualitative step forward for theory. Conceptually established in the 1950s, but largely overlooked and neglected by atomic structure theoreticians, the anomalous magnetic moment of the electron (EAMM) - beyond the $(g-2)$ QED effect on the level energy — causes an increase of the M1 transition rate by $0.45 \%$ [156,157,159]. A lifetime measurement of an accuracy better than this percentage can in principle test a leading QED correction-and there are not many QED contributions as large as this one. One can test an element of the Standard Model by a lifetime measurement that is good to, say, $0.1 \%$, and such measurements are feasible on various atomic systems nowadays. Unfortunately, there is a correspondence to the intended spectroscopic QED measurements on two-electron ions mentioned above: Atomic structure theory (apart from QED) for multi-electron ions is not up to the part (yet). When the Heidelberg EBIT group had analyzed their data and obtained error bars on the order of $0.1 \%$ [157] (which as a matter of principle need to be checked by experiments elsewhere), they found the available theoretical predictions of the lifetime (after adding the EAMM correction to all those computations that had not considered it) to scatter within an interval of about $1 \%$, for a relatively simple atomic system consisting of a closed $K(1 \mathrm{~s})$ shell, a closed $2 \mathrm{~s}$ subshell, and a single $2 \mathrm{p}$ electron. To cut the discussion short, among theorists (by now) there is little doubt about the necessity and amount of the EAMM contribution. However, it cannot presently be tested by experiment, because the much larger contribution by non-QED many-body quantum mechanics is not known with sufficient reliability. Atomic structure theory (many-body quantum mechanics) is not (seriously) in doubt, but its application by means of practical (even large-scale) computations is not good enough yet. Since testing the Standard Model (eventually) by atomic lifetime measurements seems so desirable, a few further examples will be presented below, as well as the interplay with theory in the interpretation of the data and the quest for even higher reliability.

\subsection{CNO-Like Ions}

In $\mathrm{C}$ - ( $\mathrm{Si}$-, Ge-) like ions, a noteworthy pair of intercombination transitions connects the $\mathrm{ns}^{2} \mathrm{np}^{2}{ }^{3} \mathrm{P}_{1,2}$ levels of the ground configuration with the lowest quintet level, nsnp ${ }^{3} \mathrm{~S}_{2}^{o}$. Several atomic structure details complicate the computation of the transition rate; the multiplet mixing with $J=2$ triplet levels suffers from cancellation effects in the $\mathrm{nsnp}^{3}{ }^{3} \mathrm{P}_{2}^{\mathrm{o}}$ level decays and requires the inclusion also of mixing with the $n s n p^{3}{ }^{1} \mathrm{D}_{2}^{o}$ level, and there are reasons why the fine structure splittings cannot be employed as a computational bridge as they can in other situations. Hence the triplet-quintet intercombination line has posed a challenge to theory while the lifetime data from early ion trap measurements also scattered notably. Two computations that involved convergence studies (as a function of maximum principal quantum number in the basis set) lastly agreed with each other $[160,161]$ and with the mean of the earlier experimental findings on $\mathrm{N}^{+}$ions, but a heavy-ion storage ring experiment [162] came out slightly different just beyond the mutual error bars. As Alan Hibbert jokingly noted at a conference, his second-but-last convergence step (which he had included in his publication) agreed with the new experimental result. By doing worse in procedure he could have reached seemingly perfect agreement with an experiment (then still in the making). (Hibbert's joke was a much better reaction than the anger 
that was reported to me from a theory colleague who chided me for citing her published second-order approximation computational result—which did not look so good in the end-instead of her unpublished first-order result.) With the given experimental equipment, the same transition could be (and has been) studied in only one other atomic system, the $\mathrm{O}^{2+}$ ion [166], but with a larger uncertainty that does not contribute much towards better insight.

Recent computations of C- and N-like ions [163-165] have come with extensive bibliographies, which saves me from doubling the effort. The ground configurations of ions with an open $n=2$ shell (Li- through F-like) provide prototypes for related systems with an open $n=3$ (Na- through Cl-like) or $n=4$ shell, and so on. Within the $n=2$ shell systems, there also are symmetries that link, for example, the $\mathrm{C}$ - and $\mathrm{O}$-like ions which all have ${ }^{3} \mathrm{P},{ }^{1} \mathrm{D}$, and ${ }^{1} \mathrm{~S}$ levels and similar E1-forbidden transitions between them (though the level sequence within the ${ }^{3} \mathrm{P}$ ground term is inverted in the O-like ions). This situation is attractive for systematic studies, since it increases the chance that there are ions that within the given performance envelope of a detection system have wavelength and lifetime parameters that are compatible with the apparatus. Moreover, various ion sources yield high output currents for Si, $\mathrm{P}, \mathrm{S}$, and $\mathrm{Cl}$ (less so for $\mathrm{Al}$ and typically much less so for $\mathrm{Mg}$ ). Various advantages combined at the Heidelberg heavy-ion storage ring TSR permitted high-quality lifetime measurements on C- and O-like ions of Si, P, and S (Figure 8). Lifetimes and wavelengths might have allowed some measurements also on the corresponding $\mathrm{Cl}$ ions, but the storage ring was no longer in operation then. $\mathrm{N}$-like ions have been studied as well, but the atomic structure situation is less favorable in these ions, as there are two levels that differ in lifetime by about a factor of two and decay with wavelengths that are not easily discriminated from each other by filters; a combined decay curve then consists of the superposition of both level decays and cannot be reliably disentangled with a small error bar. Measuring three instead of a single element helps with establishing systematic consistency of the data. The comparison of the data set with computations and compilations also indicates that there are sizable mistakes in certain data bases. The measurements have been described in detail [166-168] and compared there to many computations, so that we can concentrate on a comparison of the experimental data to only the best computations here (see Figure 8).

\subsection{F-Like Ions}

The ground configuration in F-like ions resembles the case of the B-like ions, except for the inverted fine structure levels. The E1-forbidden transition in the ground term is relatively prominent in dilute plasmas, and its wavelength has long since been systematized by Bengt Edlén [169]. The $J=1 / 2$ level now is the upper level of the ground configuration, and the lower statistical weight (compared to $J=3 / 2$ in B-like ions) makes for a systematically weaker M1 transition. Although lifetime measurements have been done (on Ar and $\mathrm{K}$ ions at EBITs, and on Sc and Ti ions at the TSR heavy-ion storage ring), they have not reached the same level of statistical reliability as the ones on B-like ions. The latest computations for the lowest three levels and transitions between them appears to have been done by Jönsson et al. [170]. Some of the calculated and measured lifetime values for the $2 \mathrm{~s}^{2} 2 \mathrm{p}^{5}{ }^{2} \mathrm{P}_{1 / 2}^{\mathrm{o}}$ level, the upper fine structure level of the ground term, are shown in Figure 9. 
Figure 8. Lifetime of the $2 \mathrm{~s}^{2} 2 \mathrm{p}^{2,4}{ }^{1} \mathrm{D}_{2}$ level in $\mathrm{C}$ - and O-like ions of $\mathrm{Si}, \mathrm{P}$, and $\mathrm{S}$. All measurements (full markers with error bars) have been done at the TSR heavy-ion storage ring. The Si measurements could nowadays be redone with much higher statistical reliability, if the storage ring was still available. The data are shown relative to two computations. The computations shown scatter by a few percent around the experimental data, whereas some earlier computations differed by more than $10 \%$ (see [168]).
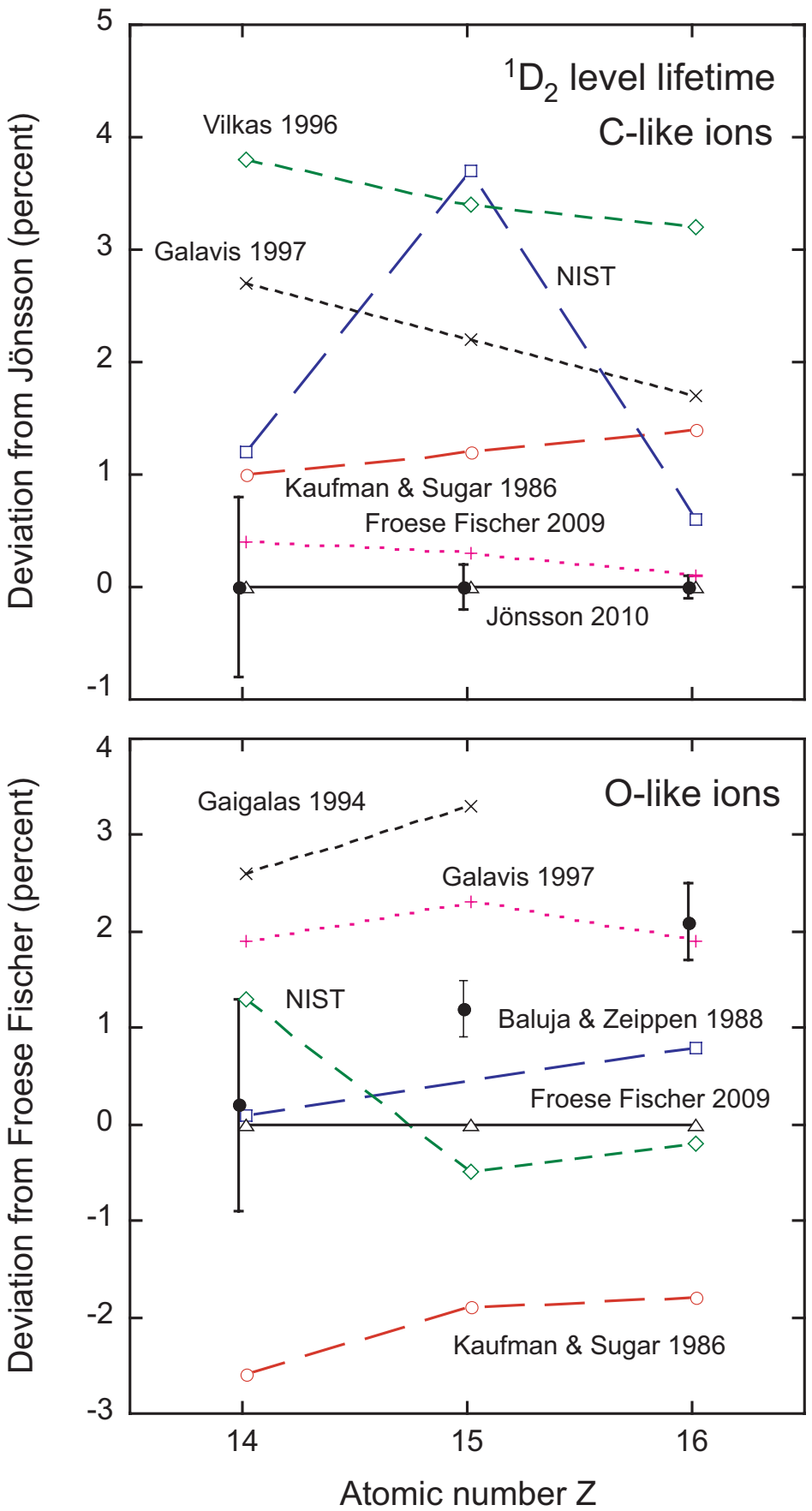
Figure 9. Lifetime of the $2 \mathrm{~s}^{2} 2 \mathrm{p}^{5}{ }^{2} \mathrm{P}_{1 / 2}^{\mathrm{o}}$ level in F-like ions from $\mathrm{Cl}$ through Ti. Measurements (full markers with error bars) have been done at an electrostatic ion trap (EST) (Ar), at the Livermore EBIT (Ar, K), and at the TSR heavy-ion storage ring (Sc, Ti). Two of the more recent computations are shown; the experimental errors are still too large to discriminate among these two computations.

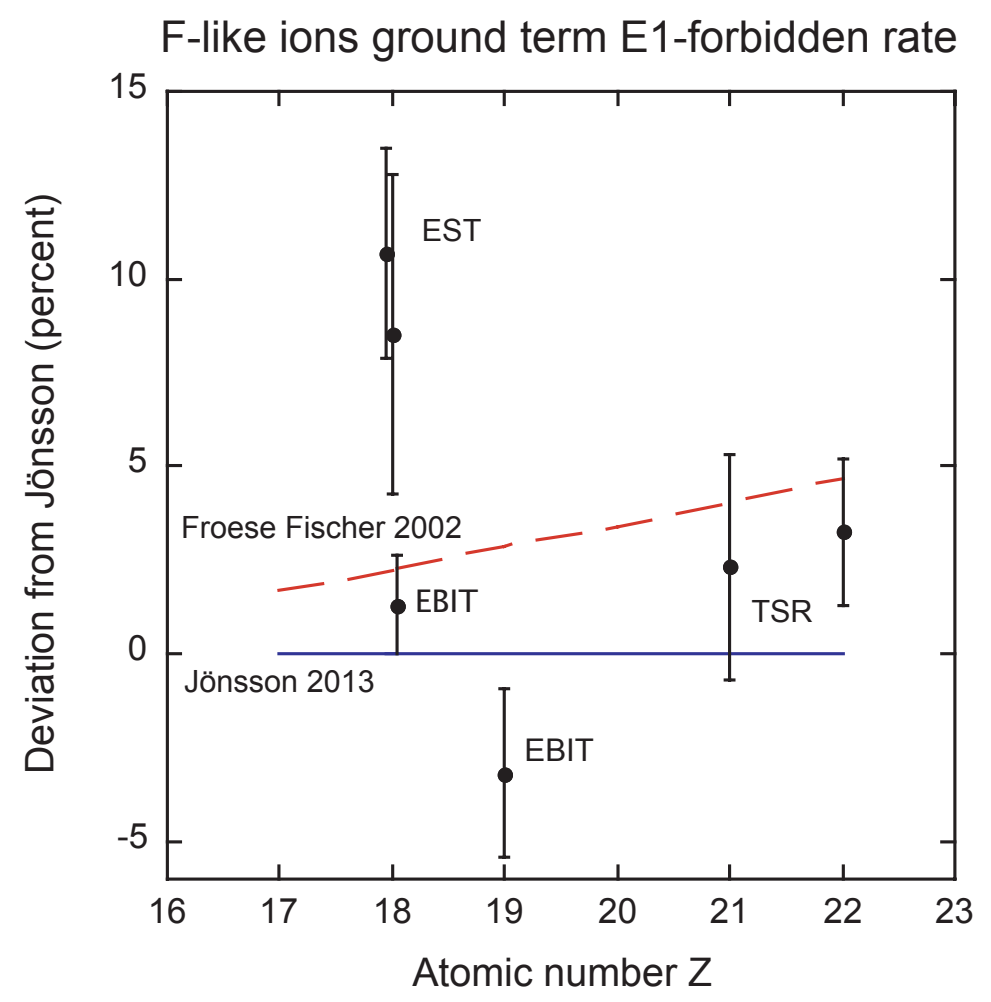

\subsection{Ne-Like Ions}

There are some similarities among the spectra of ions with electron numbers similar to those of the rare gases. A traditional approach to the computation of Ne-like ions, for example, exploits the knowledge of how to treat two-electron systems by approximating Ne-like spectra as arising from the combination of a valence electron and a vacancy in the $n=2$ shell. After a plethora of computations by various techniques, complex modern computations no longer depend on such empirical approximations. A recent paper [171] lists many of the earlier studies.

The occurrence of a resonance line and an intercombination transition $\left(2 s^{2} 2 p^{6}-2 s^{2} 2 p^{5} 3 s\right)$ with upper levels ${ }^{1} \mathrm{P}_{1}^{\mathrm{o}}$ and ${ }^{3} \mathrm{P}_{1}^{\mathrm{o}}$ has intrigued experimenters and theoreticians and perhaps has led them to assume more simplicity in Ne-like ions than is warranted. Ne-like ions have played a role in the development of certain EUV lasers (often called X-ray lasers), and when those turned out to be more complex and difficult to start than hoped for, atomic structure theory and experiment tried to find out the reasons. The practical problems with those laser experiments have been overcome by now, but Ne-like ions still pose unresolved questions. Nevertheless, there now are plenty of computations to consult, and to follow arguments on whether to maintain a given level designation when levels cross (at high $Z$ ) according to low- $Z$ systematics or whether to change the labels. Let me stick to some seemingly simple phenomena. 


\subsubsection{Levels}

Above the $2 s^{2} 2 p^{6}$ ground state the lowest excitation levels are the 36 levels of the $2 s^{2} 2 p^{5} 31$ and $2 \mathrm{~s} 2 \mathrm{p}^{6} 31$ configurations. Most of these are interconnected by $3 \mathrm{~s}-3 \mathrm{p}-3 \mathrm{~d}$ E1 transitions (EUV lines for moderately highly charged ions). Selection rules render two of the four 3 s levels metastable, leaving one of them with an M2 decay to the ground state and otherwise only M1 transitions in the same subshell. The level lifetimes consequently are very different, and the level populations must be expected to depend strongly on the environmental particle density (collision frequency). Building upon decades of work of others with various light sources, researchers at Lund (relying on laser-produced plasmas) and at Bochum (beam-foil spectroscopy) turned to Ne-like ions of iron group elements; I (as a trainee on the Bochum side) felt relieved when the results largely agreed (where the work overlapped). The process was assisted by beam-foil lifetime work at Bochum, comparing results on the $2 s^{2} 2 p^{5} 3 p$ and $2 s^{2} 2 p^{5} 3 d$ level lifetimes mostly to Fawcett's tabulations, and by Cowan code computations at Lund. As an example, most of the $2 s^{2} 2 p^{5} 3 p$ levels in Ti XIII have a lifetime on the order of $200 \mathrm{ps}$, while most of the $2 s^{2} 2 p^{5} 3 d$ levels in the same system feature lifetimes on the order of $100 \mathrm{ps,} \mathrm{experiment} \mathrm{on} \mathrm{Ti}$ (and correspondingly on S) [172,173] agreeing with the computations.

However, there are two special levels in the $2 s^{2} 2 p^{5} 3 p$ manifold, ${ }^{1} S_{0}$ and ${ }^{3} S_{1}$. The triplet level lies lower than most of the others (so the transition energy towards $2 s^{2} 2 p^{5} 3 \mathrm{~s}$ levels is lower), and hence a longer lifetime than 200 ps should be expected and was found in the Bochum experiment (which corroborated the line identification). A Russian theory paper nevertheless claimed that the line identification must be wrong, because the measured lifetime disagreed with their own prediction. However, as corroborative evidence they listed a good half dozen other computations as well which together spanned a continuum of lifetime predictions within a factor of about three (experiment fell into the middle of that range). So much for predictive reliability. The ${ }^{3} \mathrm{~S}_{1}$ level is favored in population by its statistical weight (and its lower position) over the ${ }^{1} S_{0}$ which lies well above the bulk of the $2 s^{2} 2 p^{5} 3 p$ configuration and is predicted to feature a correspondingly shorter level lifetime. Actually, this $J=0$ level has only two decay channels (to the $2 \mathrm{~s}^{2} 2 \mathrm{p}^{5} 3 \mathrm{~s}{ }^{1,3} \mathrm{P}_{1}^{\mathrm{o}}$ levels), and either of these is expected to be associated with only a weak line because of the low population of the upper level. By collaboration of the Lund and Bochum groups it was tried to identify this line pair in a sequence of elements [174]. However, in all cases at least one of the lines appeared to be blended, preventing any "closure of the loop" which would have served as a strong corroboration of correct identification. Supporting computations left choices among various possible line combinations. Two decades later, Yasuyuki Ishikawa (using his $a b$ initio MR-MP code) and Giulio Del Zanna (using scalable astrophysics modeling codes) tried again, revisited Ne-like ions, found and removed inconsistencies in earlier compilations $[175,176]$ - yet they could not conclusively place the $2 \mathrm{~s}^{2} 2 \mathrm{p}^{5} 3 \mathrm{p}{ }^{1} \mathrm{~S}_{0}$ levels. This situation is also discussed in the latest paper on such accurate computations of Ne-like ions [171], including further references to earlier ideas about peculiarities of the wave function of the ${ }^{1} \mathrm{~S}_{0}$ level.

Meanwhile, spectra recorded by the Hinode spacecraft at wavelengths near $200 \AA$ benefit from much better spectral resolution than has been available decades before in the laboratory or in solar observations from satellites, but in the low-density solar environment the relative level populations of the levels of interest are much lower than in the high-density environment of the ion-foil interaction in beam-foil 
spectroscopy. In the laboratory, EBIT spectra come closest in (low) electron density to the solar corona, with the additional benefit of a single element contributing (while in the EUV spectrum of the corona, $\mathrm{Fe}$ is dominant, but is not the only elemental species). EBIT EUV spectra of Fe, in the range of primary interest from some $170 \AA$ to some $250 \AA$, feature several lines per $1 \AA$ interval, and a considerable fraction of weak lines presently remains unidentified [177]. For computations to be useful, wavelength predictions ought to be reliable to a fraction of $1 \AA$. This level has largely been reached (with some caveats due to ongoing development and debugging work) by Ishikawa's MR-MP code when applied to systems with not too many electrons. (However, Fe VIII and Fe VII feature such a plethora of lines below $170 \AA$ that definitive detailed analyses are not yet in reach there.)

\subsubsection{Lifetimes}

The rare gases have played (and still play) important roles in the history of spectroscopy. It is amusing to see for how long the Paschen notation on the level structure has remained in use which reflects the process of "digging" into the atom from the outside, in contrast to the "rational" nomenclature of starting with the ground state of an atomic system, which reflects atomic rather than spectral structure. The resonance and intercombination lines of $\mathrm{Ne}$ and $\mathrm{Ne}$-like ions have long since been studied for their transition rates (the inverse of the lifetime of their upper levels), also by beam-foil spectroscopy [178]. The neutral rare gas atoms, seemingly easily studied, regularly have yielded several atomic lifetime results that excluded each other by their error bars. At the same time, computations have the largest problems with neutral atoms, because there the central Coulomb field is weakest in comparison to the interactions among electrons. Evidently, the neutral rare gases beyond He pose a particular challenge to experiment and theory. I want to limit my discussion to the ions of the Ne isoelectronic sequence.

By the end of the 1980s, a number of lifetime measurements had addressed the resonance and intercombination transitions in Ne-like ions up to Ar, though only with moderate accuracy. Larry Curtis then developed linearizations of the data in terms of atomic parameters such as the line strength $S$ that would be suitable for systematization and thus for providing better estimates from a sensible smoothing process. One of his tools involved a computation of $S$ for an ion with infinitely high charge $(1 / Z=0$ in a graphical representation), a reference marker that could be used as an anchor in a plot of low- $Z$ data. The beam-foil group at Lund invited him into a collaborative presentation of their extremely precise results of measurements on Ne-like ions of $\mathrm{S}$ and $\mathrm{Cl}$ [179]. These experimental results seemed much better than any other lifetime work on the isoelectronic sequence, but the new data points were in gross disagreement with the isoelectronic trend, including the interpretational scheme developed by Curtis (who then dropped the older, less precise data from his analysis [180]). Curtis therefore re-investigated the other published data and asked around for new measurements. He would have liked an explanation for the inconsistent data set on Ne I, too, but that did not happen. Träbert re-analysed old Bochum measurements and extended the local list of results up to $S$ [181]. All data curves showed hefty cascades; at very low $Z$, the $3 \mathrm{~d}$ level lifetimes were close to but shorter than that of the $3 \mathrm{~s}$ triplet level (giving rise to a growing-in cascade), for $\mathrm{Al}$ and $\mathrm{Si}$ the 3 s triplet and several $3 \mathrm{~d}$ level lifetimes were too close to each other for a meaningful analysis, and only for $\mathrm{P}$ and heavier elements the curve tail was dominated by slow cascades. Hence the curve structure could be understood by cascade models, but no high accuracy measurements were possible. Nevertheless, the data trend clearly suggested serious errors (by several 
standard deviations) in the seemingly precise Lund data on $\mathrm{S}$ and $\mathrm{Cl}$. Half a year later, another publication from Lund [173] changed their sulphur lifetime results part of the way to where Curtis' analysis had long since pointed to. The background story of the mistakes is not to be told here, but a take-home lesson is that even highly qualified researchers are not immune against bouts of hubris. For atomic physics, we still do not have highly precise and accurate lifetime data on low- $Z$ Ne-like ions, but we now know why: in beam-foil spectroscopy at least, the cascade situation does not permit a better measurement. By the way, the earlier and less precise measurements on elements $\mathrm{Cl}$ and beyond that had been assumed to be too poor to be included in analyses any longer when the (seemingly) extremely good-looking data were published have returned to the isoelectronic analysis since, because they were consistent with the overall trend after all. A recent computation [171] also reviews the data base and claims good overall agreement, including the aforementioned $3 \mathrm{p}$ and $3 \mathrm{~d}$ level lifetimes (although in $\mathrm{Cl}$ there is some notable mismatch ... with those ominous Lund data).

At a very much higher nuclear charge, $Z=55$, Ne-like $\mathrm{Cs}^{45+}$ ions feature $3 \mathrm{~s}$ level lifetimes in the femtosecond range. In an electron beam ion trap, the population of the $3 p$ and $3 \mathrm{~d}$ levels is rather small compared to $3 \mathrm{~s}$, which alleviates cascade worries. The ion sample is stationary in such a trap, the ion cloud can be cooled so that the thermal (Doppler) line broadening shrinks below the lifetime broadening, and the instrumental line width can be taken care of by simultaneous studies of longer-lived levels [182]. The lifetime thus measured from the lifetime broadening of the resonance line has come out close to expectation, but more such quality data ought to be recorded for a sensible test of atomic structure theory in the high- $Z$ range.

\subsubsection{Line Ratio}

At first glance, the pair of strongest EUV lines in coronal spectra of various stars (including Sun) might seem too closely related to the aforementioned resonance and intercombination transitions to merit particular attention. The two lines of Fe XVII have been dubbed line $3 \mathrm{C}\left(2 \mathrm{p}^{6}{ }^{1} \mathrm{~S}_{0}-2 \mathrm{p}^{5} 3 \mathrm{~d}{ }^{1} \mathrm{P}_{1}^{\mathrm{o}}\right.$, $15.014 \AA)$ and line 3D $\left(2 \mathrm{p}^{6}{ }^{1} \mathrm{~S}_{0}-2 \mathrm{p}^{5} 3 \mathrm{~d}^{3} \mathrm{D}_{1}^{\mathrm{o}}, 15.261 \AA\right)$, respectively. One might expect that the strongest features in a spectrum ought to be understood first and best. However, the line intensity ratio 3C/3D was regularly calculated as larger than what was seen in low-density plasmas on Earth or in stellar coronae in outer space. An exception was the NIST EBIT; their early result was in the theory ball park, and it has taken years and many corrections to approach the range in which many systematic experimental studies at the LLNL EBIT had been long before-plus all those observers of tokamak plasmas or the stars. Since neither experimenters nor theoreticians found fault with their own ways and results, the systematic discrepancy between experimental and computed line ratios was blamed on a variety of collisional-radiative processes in distant light sources that one could diagnose by the size of the discrepancy. However, Greg Brown's extensive and tedious systematic studies at the LLNL EBIT [183,184] agreed with the stars, but could not suffer from the same collisional effects. Moreover, Ming Feng Gu's computations by his FAC code contradicted those computations that had claimed they could match the observations after all. Work by others eventually suggested that the NIST EBIT problems may have arisen from less well controlled run conditions of their EBIT than the experimenters had believed (causing a blend with a lower charge state ion to matter) and so on. 
A digression: Trying to do science, a scientist may well encounter social phenomena that one would hope not to contaminate the community. Among these are camps with followers and entrenchments against others that all believe and claim to be motivated by science. In the present context, I know of Livermore colleagues who encountered at least one referee (but then repeatedly) who wanted to discourage them from applying more experiments (or modeling computations) to this very problem, since - in the strongly expressed view of the referee - a theoretician (let us call him XG) had finished off the problem for good-and anyway the NIST EBIT experimental results agreed with that material. One of the colleagues, seeing his chances for successful grant applications dwindling, because he could no more publish in the key journal, left the field in disgust. The trust this referee had put into one theorist and one institute is, mildly put, surprising. The referee(s) had conveniently overlooked the fact that XG had produced three papers in two years, with different, partly incompatible, sets of results. Which one of those computations was the definite solution to all problems? Why trust computations that change so quickly? How come the NIST EBIT results had to be corrected in the end so that nowadays the "world view" on this topic is where the Livermore group data and computations had been all the time? Referees of a strange ilk and with deep-rooted prejudices can be major obstacles in the process of developing experiment and computations in dialogue.

Back to the scientific topic of the 3C/3D line ratio: Eventually the evidence pointed away from those collisional effects and to theory instead. However, for a decisive test, the experiment could be made even cleaner than before: a collaboration of Heidelberg and Livermore EBIT experts and their associates prepared a sample of $\mathrm{Fe}^{16+}$ ground state ions (with some lower, but no higher charge state ions in the trap) in the usual way (by electron beam ionization and excitation). The decisive excitation of the $3 \mathrm{C}$ and 3D lines was effected by selective photo-excitation (Figure 10), using the LCLS X-ray laser at the Stanford Linear Accelerator Center. The observed line ratio clearly confirmed the earlier experimental work (mostly done at the Livermore EBIT) and was at variance with all computations [186,187]. When the report on this finding was submitted to a high-profile learned journal, a journal referee suggested that "two weeks of theory work should sort out the problem", overlooking the world-wide, many-group, many-technique, many-year effort already spent on the task. The mixing of the two upper levels may be dominated by the two principal states given in the labels, but the computations included many other $J=1$ levels as well, as well as computations with extremely large basis sets, and nothing has worked out yet to bridge the discrepancy. Atomic structure computation in this case appears to be at significant odds with nature. If the reasons for the discrepancy were known, they might well have been sorted out by now. Speculation points to the actual wave functions, which might have properties that have been overlooked so far. There is much more work to be done!

\subsection{Na-Like Ions}

The D absorption lines in the solar spectrum have been labelled by Joseph Fraunhofer near the beginning of the $19^{\text {th }}$ century; half a century later the corresponding emission lines in the spectrum of a flame were identified first with table salt and then with sodium, thus helping to establish spectral analysis as a laboratory tool and with finding out what the stars consist of. The strong $\mathrm{Na}$ I resonance line doublet has intrigued theorists and experimenters, and nowadays observations and computations [100,101] of 
the spectra of Na-like ions reach all the way up to $U$ [188,189]. For high values of $Z$, the fine structure interval between the two D lines (the $3 \mathrm{p}^{2} \mathrm{P}$ "fine structure" splitting) is much larger than the excitation energy of the $3 p_{1 / 2}$ level. Recent measurements at the NIST electron beam ion trap have tended to a wide range of elements in the upper half of the periodic table [190,191]; in those papers, the new results along with the many earlier data points compare well to the 20 years old Blundell computations.

Figure 10. Level scheme of the lowest 37 levels in the Ne-like ion $\mathrm{Fe}^{16+}$. The two arrows point to the $2 \mathrm{p}^{6}{ }^{1} \mathrm{~S}_{0}-2 \mathrm{p}^{5} 3 \mathrm{~d}{ }^{1} \mathrm{P}_{1}^{\mathrm{o}},{ }^{3} \mathrm{D}_{1}^{\mathrm{o}}$ transitions discussed in the text and illustrate the resonant photo excitation experiment performed at the LCLS X-ray light source [187].

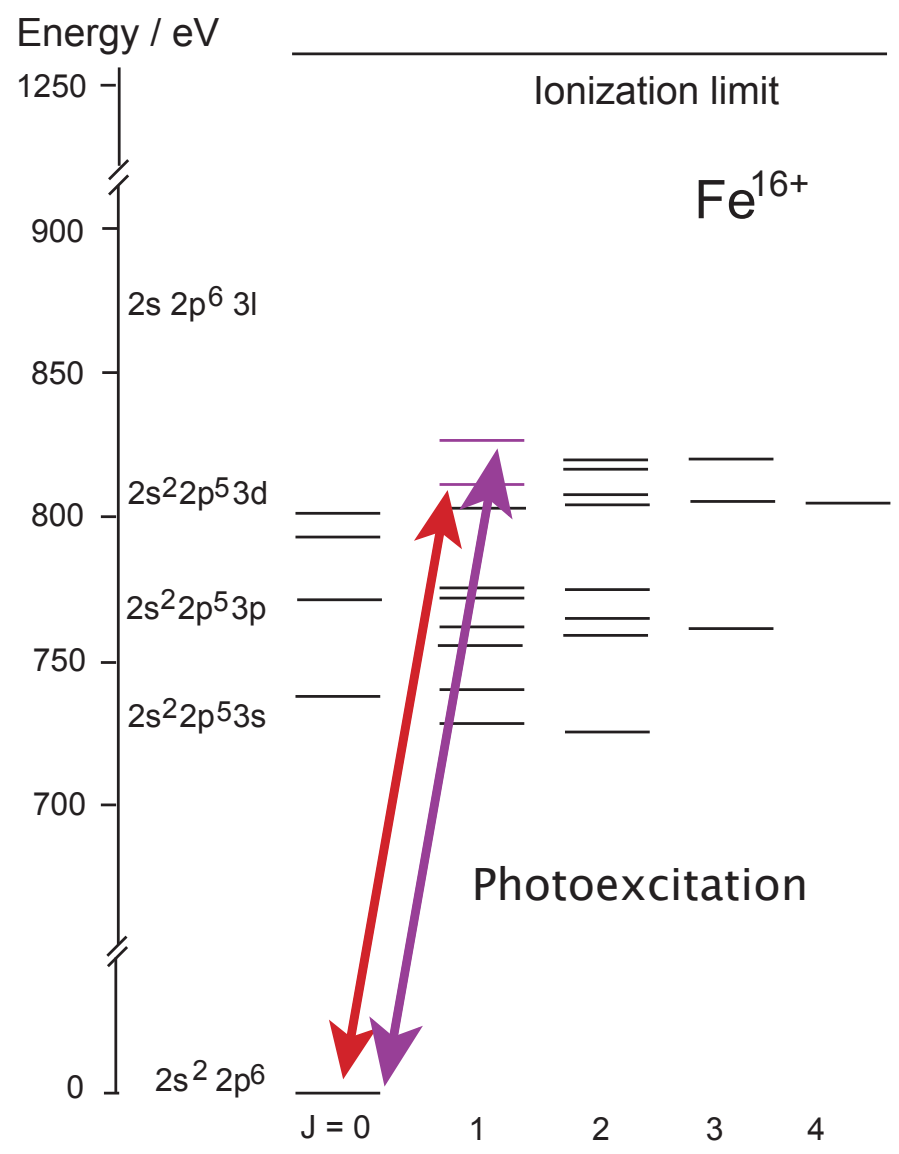

While EBITs are very useful light sources for spectroscopy, they are much less used for atomic lifetime measurements owing to detection and signal timing problems in many ranges of the spectrum. This may change some day when EUV lasers become more readily accessible and can be used to selectively photo-excite ions in an EBIT. For multiply charged Na-like ions so far only foil-excited ion beams have been employed (for the most recent papers on the subject, see [34-36]). Within their 5 to $10 \%$ error bars, the results all match computation.

\subsection{Mg-Like Ions}

Just as the Na-like ions with their $n=3$ valence electron are largely analogous to the Li-like ions with their $n=2$ valence shell, a general assumption is that Mg-like ions are not so much 
more difficult to calculate than Be-like ions. However, Vilkas et al. [192] have intercompared four computations reported in the literature up to high values of $Z$, and were surprised by some massively different predictions of the isoelectronic trends of the $3 \mathrm{~s} 3 \mathrm{p}{ }^{1,3} \mathrm{P}_{1}^{o}$ level energies (the resonance and intercombination transitions) - see their Figure 1. The authors of those discordant computations are known for good work in many other cases. It has not transpired to me what might explain why several computations went so far astray. However, the case reminds us customers of atomic structure computations to remain cautious also about computations performed by renowned experts. On the other hand, dedicated MBPT, MR-MP, or RCI computations nowadays almost certainly will yield results close to experiment. What went wrong in those computational surveys?

The valence electron in $\mathrm{Mg}$-like ions is ascribed a penetrating orbit; hence the nuclear charge the electron experiences is less well shielded by the inner electrons than otherwise. The result is a shorter level lifetime than in a Be-like ion of similar ion charge. For example, the $2 s^{2}-2 s 2 p$ intercombination transition in $\mathrm{B}^{+}$reveals an upper level lifetime of about $100 \mathrm{~ms}$ [193] while the corresponding decay curve of the $\mathrm{Al}^{+} 3 \mathrm{~s}^{2}-3 \mathrm{~s} 3 \mathrm{p}$ intercombination transition features a lifetime of about $0.3 \mathrm{~ms}$ [193,194]. The latter lifetime is close to the short-lifetime limit of practical work with a heavy-ion storage ring, which unfortunately precludes further such measurements along the isoelectronic sequence. Nevertheless, it is a comforting thought (for experimenters) that two very different experiments (one using a radiofrequency ion trap, the other a heavy-ion storage ring) have yielded practically identical lifetime values-which, by the way, in this case theory is struggling to reproduce.

The measurement situation for the $3 \mathrm{~s} 3 \mathrm{p}{ }^{1} \mathrm{P}_{1}^{o}$ level is very similar to that encountered in the $\mathrm{Be}$ isoelectronic sequence, with the same cascade pattern and even a few additional cascades. It is difficult to muster equipment for observing and measuring all the important cascades that play a role after the excitation of a fast ion beam by passing through a solid foil. Quite some time ago the alternative of a population and cascade model has served beam-foil spectroscopy well—with the accordingly somewhat limited accuracy of the results [19]. However, lifetime uncertainties of about $10 \%$ are quite respectable after all, and not (yet) easily surpassed by theory (computation) for arbitrary levels.

\subsection{Al-Like Ions}

Al-like ions have been of interest to spectroscopy in many aspects, the line intensity anomalies resulting from level mixing are an example already mentioned above [150]. In the story of the identification of intercombination transitions in the EUV spectrum of the solar corona $[195,196]$ from beam-foil spectra recorded in the accelerator basement, the $3 s^{2} 3 p^{2} \mathrm{P}^{\mathrm{o}}-3 \mathrm{~s} 3 \mathrm{p}^{2}{ }^{4} \mathrm{P}$ lines have played a key role, because their wavelength and intensity pattern was so characteristic. Mg-like spectra of iron-group elements have a single intercombination transition, which may be bright (and was known from tokamaks), but a single line is difficult to identify. Al-like spectra, however, feature five lines in a reasonably well predictable wavelength and intensity pattern, a fingerprint. With increasing nuclear charge, the fine structure splitting drives the lines apart, but some of them remain close enough to each other to fall into a wavelength range covered by a single position sensitive detector. In this way the lines have been followed in beam-foil spectra up to $Z=79(\mathrm{Au})$, and some of the lifetimes have been measured [197]. For the Al isoelectronic sequence, the high- $Z$ trend of Huang's MCDF computations 
was found closer to experiment than similar computations for the other isoelectronic sequences done in the same period. This was likely a chance success, related to a systematic trend: ions with fewer electrons showed one sign of deviation and ions with more electrons deviated to the other side. Reliable $a b$ initio computations of those sequences have appeared only some two decades after Huang's original work (see, for example, [192]).

At lower $Z$ one might expect that all relatively low-lying levels of Al-like ions are known. Santana et al. [198] have calculated (using the MR-MP formalism) the lowest 40 levels of Al-like ions in the iron group $(\mathrm{K}(Z=19)$ through $\mathrm{Ge}(Z=32))$ and compared the results with the NIST data base. For elements from $\mathrm{Ti}$ through $\mathrm{Ni}$, almost all of the levels of the $3 \mathrm{~s}^{2} 3 \mathrm{p}, 3 \mathrm{~s} 3 \mathrm{p}^{2}, 3 \mathrm{~s}^{2} 3 \mathrm{~d}, 3 \mathrm{p}^{3}$, and $3 \mathrm{~s} 3 \mathrm{p} 3 \mathrm{~d}$ configurations were already known, whereas at a little lower or higher $Z$, the knowledge was and is rather patchy. It is a pleasure to see how closely these ab initio computations match measurement, fill gaps, and suggest revisions with almost spectroscopic accuracy. Interestingly, for several ions certain groups of levels differ significantly more $(0.5 \%$ to $1 \%$ ) from computation than the majority of others (less than $0.05 \%$ ), suggesting that the experimental data quality may have been poorer in these cases (there also were apparent misidentifications). It might be possible to close some of the data gaps by going back to the original data recordings and now use the guidance provided by good computations. The quality of the MR-MP computations also shows in the fact that it was possible to calculate (by $a b$ initio means) the ground state fine structure in Fe XIV correctly [199]. A comparable set of computations of Al-like ion levels and transition rates has since been executed by C. Z. Dong's group at Lanzhou, using the GRASP2K code, but I have not seen any published results.

Encouraged by the success of the atomic structure work, MR-MP computations have been used to address the decay channels of those levels the lifetimes of which are largely determined by spin-forbidden and/or E1-forbidden decays, that is the $3 \mathrm{~s}^{2} 3 \mathrm{p}^{2} \mathrm{P}_{3 / 2}^{\mathrm{o}}$ level, the three $3 \mathrm{~s} 3 \mathrm{p}^{2}{ }^{4} \mathrm{P}_{J}$ levels, and the $3 \mathrm{~s} 3 \mathrm{p} 3 \mathrm{~d}^{4} \mathrm{~F}_{9 / 2}^{\mathrm{o}}$ level. The latter has long been overlooked as the origin of a cascade with almost the same lifetime that might spoil lifetime measurements of the $3 \mathrm{~s}^{2} 3 \mathrm{p}^{2} \mathrm{P}_{3 / 2}^{\mathrm{o}}$ level. The likely influence of this cascade is a lengthening of the apparent lifetime, and the effect would be most pronounced in an environment in which the cascade level is more highly populated. Such a pattern is, in fact, apparent in the experimental data [200-205], as has been discussed in more detail elsewhere [12]. In Fe XIV the cascade lifetime (calculated by FAC [185] and MR-MP codes) is too close to the primary to be discerned in the decay curve of the $3 \mathrm{~s}^{2} 3 \mathrm{p}^{2} \mathrm{P}_{3 / 2}^{\mathrm{o}}$ level. However, the cascade lifetime scales differently from the primary decay and the cascade has thus been confirmed in heavier ions nearby [206]. The cascade contribution is smallest in the excellent vacuum of an EBIT, but not really negligible. The most accurate lifetime measurement on Fe XIV [205] is, indeed, on the slow side of theoretical expectation, as would be in the (neglected) presence of the cascade. To repeat a point mentioned above: There also is a need to take the EAMM correction to the M1 transition moment into account, and when one does that, the disagreement of the lifetime measurement result with theory increases and actually becomes quite sizeable (see Figure 11).

Similar 3d cascades plague the lifetime measurements of ground configuration levels also in Si-, P- and S-like ions [206]. However, the 3d levels, especially those of high total angular momentum $J$, have hardly been covered in systematic computations and thus probably are also missing from most collisional-radiative models. 
Figure 11. Timeline of the transition rate of the ground state fine structure transition in the Al-like ion $\left(\mathrm{Fe}^{13+}\right.$ of iron. The four experimental data points result from two experiments with an electrostatic ion trap and from two that used EBITs. In the latest one [205] the error bar is smaller than the symbol size. The vertical red line (left one of the pair) shows the result of naive theory combining the experimental transition energy and the single-configuration picture line strength. The accompanying dark blue line indicates the same expectation, but corrected for the EAMM contribution from quantum electrodynamics (QED). Note that the best experimental data point does not agree with what presently is seen as a correct theoretical prediction. All of the non-ab initio computations fall close to these two lines, because they have been adjusted to the experimental transition energy. Of the four $a b$ initio computations only one (MR-MP) [199] coincides with the range of successful experiments by merit (and still misses the EAMM correction). The computational result provided by Huang [67] coincides with experiment as well, but by comparison with other atomic systems one has to conclude that these computations are not accurate enough to call this more than a chance agreement. (Figure adapted from Figure 1 of [198], which includes a list of all the references.)

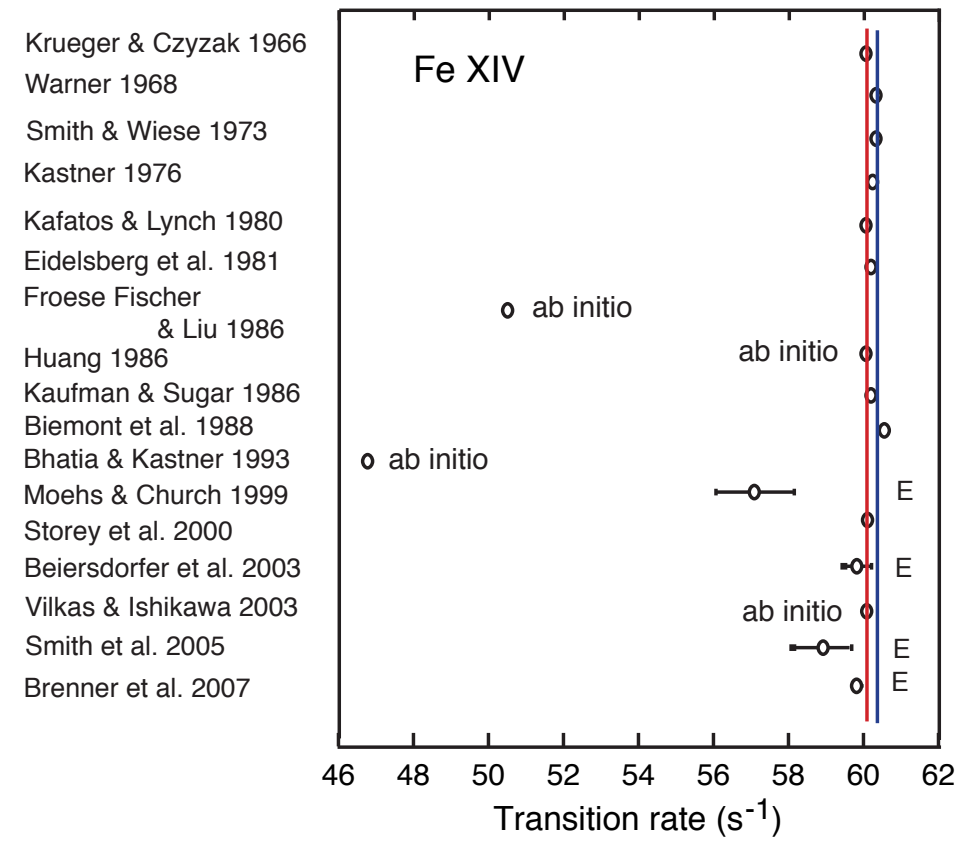

\subsection{Si-Like Ions}

In Si-like ions, the lowest configurations are $3 s^{2} 3 p^{2}, 3 s 3 p^{3}$, and $3 s^{2} 3 p 3 d$. The lowest $3 s 3 p^{3}$ level is a quintet level, ${ }^{5} \mathrm{~S}_{2}^{\mathrm{o}}$, which is of atomic structure interest for reasons similar to the $2 \mathrm{p}^{3}{ }^{5} \mathrm{~S}_{2}^{\mathrm{o}}$ level in C-like ions discussed above. At the beginning of the isoelectronic sequence, the two intercombination decays of this level have been known for quite some time. Beam-foil spectroscopy has helped to locate the corresponding lines in the EUV spectra of iron group elements and thus also in the solar corona [195,196]. However, in Si-like Fe, one of the two lines is blended with a strong He line in the corona, and therefore the accuracy of determining the level position and the lower level fine structure splitting is jeopardized. Elements heavier than $\mathrm{Fe}(Z=26)$ are less abundant in the solar corona; 
consequently the reliability of extrapolations of the atomic structure data suffer. Given that the decays of long-lived levels are not very bright in time-resolved observations such as by beam-foil spectroscopy, and that there are many unidentified lines in the same wavelength range, certain line identifications may be more tentative than one would like. A Swedish-Chinese collaboration assumed the upper hand in the identification process of the intercombination transitions, because they had wavelengths of four elements on a straight-line plot which they saw as proof by isoelectronic trend [207]. However, the newly claimed trend was much steeper than that of the (many more) lower- $Z$ data and also incompatible with any typical atomic structure computational trend. In spectra with many unidentified lines one may occasionally find a sequence of several lines that fit to a common reference by chance, but atomic data analysis would not have gotten to its present state of achievement by chance. Renewed analyses of experimental data [208] as well as extensive MR-MP computations up to $\mathrm{Au}(Z=79)$ [209] have since corroborated the orthodox data interpretation.

Si-like ions have another term that has been difficult to handle, $3 \mathrm{~s}^{2} 3 \mathrm{p} 3 \mathrm{~d}^{3} \mathrm{~F}^{\mathrm{o}}$, with three fine structure levels of total angular momentum values $J=2,3,4$. The $J=2$ level is nothing special and well enough known since a long time. However, the selection rules for radiative transitions render the $J=3$ level much longer lived than the typical neighbors, and the $J=4$ level is even metastable, without any E1-allowed decay channel. Biémont's 1986 Cowan code computations [210,211] tried to place the $3 \mathrm{~s}^{2} 3 \mathrm{p} 3 \mathrm{~d}^{3} \mathrm{~F}^{\mathrm{o}}$ term and estimated the fine structure splittings. Guided by these estimates, Träbert identified candidates for the $3 \mathrm{~s}^{2} 3 \mathrm{p}^{2}{ }^{2} \mathrm{P}_{2}-3 \mathrm{~s}^{2} 3 \mathrm{p} 3 \mathrm{~d}{ }^{3} \mathrm{~F}_{3}^{\mathrm{o}}$ transition in delayed beam-foil spectra, that is, he used the predicted nanosecond lifetime characteristic to enhance the contrast to the surrounding lines which should have lifetimes in the few-dozen to few-hundred picosecond range. The beam-foil observations led to line identifications in spectra of the solar corona that had wavelengths already known with higher accuracy [212]. Again guided by Biémont's calculated fine structure intervals one could then obtain estimates of the $3 \mathrm{~s}^{2} 3 \mathrm{p} 3 \mathrm{~d}{ }^{3} \mathrm{~F}_{4}^{o}$ level positions. This level should feature a lifetime in the millisecond range (for many iron group elements), which is much too long for beam-foil spectroscopy, but perhaps measureable in an ion trap. About a decade later, Vilkas and Ishikawa began to apply their MR-MP code to Si-like ions [213,214], and they noted gaps in the NIST data base as well as discrepancies of literature data on the $3 \mathrm{~s}^{2} 3 \mathrm{p} 3 \mathrm{~d}^{3} \mathrm{~F}^{\circ}$ term compared to their computations. The latter corroborated Träbert's aforementioned analysis. However, in spite of this progress, nobody has positively identified any of the decay branches of the ${ }^{3} \mathrm{~F}_{4}^{\mathrm{o}}$ level yet. Träbert et al. [206] have discussed how the influence of the level decay shows in the decay curve of the $3 s^{2} 3 p^{2}{ }^{1} D_{2}$ level, but the decay can take many paths, each of which is elusively weak. The latest dedicated computations on this problem [215] have yielded predictions many of which seemed consistent with weak lines in the solar spectrum at the time, but continuing work with the Hinode spacecraft data have led Giulio Del Zanna to state that the comparison with the solar data should no longer be considered conclusive [216,217]. Of course, the solar corona is a low-density environment, and such levels will not be populated very much, leaving all decay channels weak. We thus have a situation in which an experiment at a heavy-ion storage ring clearly demonstrates the cumulative effect of the weak decay channels none of which has individually been observed. (The heavy-ion storage ring also provides a low-density environment, except for the stripper foil in which the high charge state ions are produced. That is the decisive element that boosts the metastable level 
population so that the contribution is visible and that at the same time precludes a clean measurement of the ground configuration level lifetime.)

Again, as in Fe XIV, the 3d cascade in Fe XIII has about the same lifetime as the 3p level of primary interest. Experimental information can be gained from ions slightly offset along the isoelectronic sequence, because the isoelectronic scaling of the decay paths is different. In such a situation, good transition rate computations are very helpful, even as the measurement does not test the computations with high precision. Unfortunately there are only very few published computations of the $3 \mathrm{~d}$ level lifetimes. The crossing of the lifetime trends of $3 \mathrm{p}$ primary and $3 \mathrm{~d}$ cascade near Fe is evident in a step function of the experimental lifetime data [206] (relative to any one of the computations). Over all, the scatter of the calculated values for the $3 p$ level lifetimes in Si-like ions is considerable (on the order of $\pm 10 \%)$. The MR-MP computations come out best of the ab initio ones, but not as close as some scaled ones. The latest computations, by Charlotte Froese Fischer [218], promise progress towards accurate results, but they address only three elements ( $\mathrm{Fe}, \mathrm{Co}, \mathrm{Ni})$.

There also is an interesting statement in that last paper that for me illustrates a difference in world view between experimenters and theoreticians (of course, the quotation is out of context and not intended to be read the way I do): "Experimental lifetimes may be either shorter or longer but generally are within $10 \%$ of ab initio." For this theoretician, the measure of things appears to be the result of an ab initio atomic structure computation, and from there one looks around and probably finds (reasonably well) measured data in the neighborhood. For me as an experimenter, the goal is to do an experiment that comes as close to the (unknown) correct result (nature) as intrinsically possible. Then I can contemplate what a computation yields that mimics a correct description of nature from first principles (ab initio), or a computation by whatever means that includes adjustments which "usually yield some sort of agreement with measurement". The goals are to know the correct result (factual knowledge for whatever purpose) and to improve the ab initio computation (a physical description of nature) until it matches the correct result, a process that (so we hope) yields (physics) insight. Thus ab initio computations per se are of limited interest, unless they are intellectually tied to a target. (Under that condition, adjustable computations (such as [219]) may be and have been helpful, but only ab initio computations are meaningful.)

Recently the venerable non-relativistic CIV3 code has found its way also to Egypt, and it has been applied to two sections of the Si isoelectronic sequence [220,221]. I would be surprised if the range of the latter paper $(Z=30-33)$ was not served better by relativistic computations. Most of the aforementioned computations have been limited to rather low lying levels in the $n=3$ shell. Vilkas and Ishikawa [222] apply their MBPT computations also to $n=4$ levels. Much more needs to be done on both sides, experiment and computation.

\subsection{P-Like Ions}

The $3 \mathrm{~s}^{2} 3 \mathrm{p}^{3}$ ground configuration of P-like ions features a ${ }^{4} \mathrm{~S}_{3 / 2}^{\mathrm{o}}$ ground level, two relatively short lived ${ }^{2} \mathrm{P}^{\mathrm{o}}$ levels and two relatively long lived ${ }^{2} \mathrm{D}^{\circ}$ levels. Computations of this system have evolved from semi-empirically adjusted to ab initio [223-227]. The wavelength and lifetime pattern has advantages and drawbacks for the measurement of various ions of the iron group, as might be expected. Surprising to 
me is that the various computations have problems with the individual levels of the same configuration. Some of the results of the MR-MP computations differ from the trend of the others at low $Z$, and the results also differ from measurement more than the others (by up to a quarter) for the longest-lived of the ground configuration levels (see [206]), the ${ }^{2} \mathrm{D}_{5 / 2}^{o}$ level, while the experiment seems straightforward in this case. This observation raises questions about code debugging, considering that the same code has often produced excellent results in other circumstances. With the P-like ions, the MR-MP computations are among the very few that have been used to address the problem of a few long-lived $3 \mathrm{~d}$ levels, and there the results seem fine.

\subsection{S-Like Ions}

Traditional, mostly scaled, computations [228-231] have approximated the observed spectra of S-like ions, but near-spectroscopic accuracy by ab initio computations has only recently been approached [232]. Similar to the corresponding C- and O-like atomic systems, there are structural similarities between $\mathrm{Si}$ - and S-like ions. The various spectra of Fe ions with an open $n=3$ shell are being re-analyzed by Del Zanna and colleagues [233,234] with the help of adjustable computations and solar spectra (mostly those recorded by the Hinode spacecraft) and with the aim to understand the very same solar spectra in detail. As a long-term project, the CHIANTI data base [280,281] is continually upgraded, and meanwhile it has surpassed the quality of the NIST data base [279] in the EUV spectral range. However, the two data bases have been set up with different intentions: The NIST data base aims at recording data for all elements, while CHIANTI aims at understanding the solar spectrum, which implies that elements of different abundance are of different interest to solar research.

As concerns the E1-forbidden decays in the ground configuration of S-like ions, a striking contrast to Si-like ions appears. Instead of a single slow $3 \mathrm{~d}$ cascade there are six long-lived ones, widely spaced in lifetime [206]. ('Long-lived' meaning the millisecond range when most other levels in the neighborhood feature picosecond lifetimes.) They have been predicted by MR-MP computations, and they are so strong and so numerous that the $3 \mathrm{~d}$ cascades seriously affect any evaluation of $3 \mathrm{p}$ ground configuration decay curves. Any meaningful collisional-radiative model of S-like spectra has to include the $3 \mathrm{~d}$ levels, but hardly any large-scale computation is available that does.

\subsection{Cl-Like Ions}

The ground state of Cl-like ions is only one vacancy away from the closed-shell Ar-like system with 18 electrons. Nevertheless, the atomic structure of the excited ions is complex enough to be demanding for computations. Various survey computations have addressed the isoelectronic sequence (see references in [235] and in the specialized publications cited below). Fe X, the Cl-like spectrum of $\mathrm{Fe}$, is rather prominent in the quiet solar corona. Consequently, it has been studied in observational detail [236,237] and by a large-scale ab initio MR-MP computations [238].

While $\mathrm{Fe} \mathrm{X}$ as a topic of a single-spectrum presentation seems well justified by its coronal prominence, there are other studies of individual Cl-like ions which reflect the respective authors' efforts, but have ever so little predictive value. In fact, one of the publications states (on the single atomic lifetime value for which an experimental result is available for that particular ion species) that the calculated 
result is close to results of this measurement and another computation, and that yet other computations had other (cited) results. There is no explanation, no factual discussion, just the cited rather pointless statement. The results of this computation have been obtained with operators in length and velocity form, and the two results differ by almost a factor of two. The computational uncertainty is not discussed. No, this is not a paper from the dark ages, this is a 2007 publication (not cited here on purpose), using a (good) code (CIV3) as learned by the lead author decades earlier, but it has not been put to work to gain insight. Lest the reader should get me wrong: this paper reported on an apparently good and valid calculation - there are much worse. (By the way, the simply mentioned "other results" scatter by a factor of three or more, as will be discussed below.)

What should be best calculable in Cl-like ions are the lowest levels and the transition rates between them. The fine structure transition in the ground configuration is, of course, technically similar to that in the F-like ions, with a $J=1 / 2$ upper level (low statistical weight, and hence a low line intensity). The transition rate is dominantly M1 with a small E2 admixture. In Fe X, this line is the 'red coronal Fe line' which unfortunately lies just outside the good working range of efficient low-noise photomultiplier tubes that have been optimized for the visible range. The level lifetime in Fe X has nevertheless been measured at the Heidelberg EBIT [205]. From measurements of the next heavier elements at the Heidelberg heavy-ion storage ring TSR not only three isoelectronic lifetime results (on Co XI, Ni XII, and $\mathrm{Cu}$ XIII) have been obtained, but also — by extrapolation—another one for Fe X [239]. The results (with uncertainties below 1\%) are close to theoretical expectation (including the EAMM correction), but - just as for the B-, F-, and Al-like systems discussed above-the non-QED computations are not accurate enough to actually test the complete computational atomic structure package.

There is another test bench in Cl-like ions, and this one is unique so far (in the course of this discussion; there must be many atomic systems with similar features). The lowest excited level above the ground term is the displaced level $3 \mathrm{~s} 3 \mathrm{p}^{6}{ }^{2} \mathrm{~S}_{1 / 2}$ with a single electron outside closed subshells and only two decay branches to the two fine structure levels of the ground term $3 \mathrm{~s}^{2} 3 \mathrm{p}^{5}{ }^{2} \mathrm{P}^{\mathrm{o}}$ where there is a single vacancy difference from a rare gas electron configuration. This arrangement offers a variety of tests, the simplest of which would be the level energy of the $3 \mathrm{~s} 3 \mathrm{p}^{6}{ }^{2} \mathrm{~S}_{1 / 2}$ level, which is easy to do and only requires the transcription of published tables into work sheets. Let us try something a step more advanced that involves transition rates. The sum of the two transition rates towards the ground state yields the inverse of the level lifetime, and the ratio of the two transition rates is the line ratio. In moderately high charge states, the line ratio is expected to follow a smooth isoelectronic trend, and when using a grazing incidence spectrometer for the observation, no gross discontinuities of the reflectivity or detection efficiency are expected to occur over the narrow wavelength interval of this line pair. A beam-foil study has found agreement among the predicted and measured line ratios within about $10 \%$ and agreement of the measured lifetime with some theory at the same level [240]. However, the computations available at the time spanned a factor of two between the various absolute lifetime / transition rate predictions. (The mean of all predictions would have been fine.) Also, the isoelectronic trends of the predictions differed notably. There have been several new computations since then that have confirmed the same picture (with less scatter), but without new lifetime measurements in the Fe group elements (the Bochum experimental set-up has since been forced out of the laboratory), no new insight is expected for those moderately high charge state ions in the near future. 
Figure 12. The lifetime $\tau$ (scaled by the ion core charge $\zeta$ ) of the $3 \mathrm{~s} 3 \mathrm{p}^{6}{ }^{2} \mathrm{~S}_{1 / 2}$ level lifetime in Cl-like ions. Only a selection of experimental data (full squares) and computational results (open symbols for $\mathrm{Ar}, \mathrm{Ca}$ and $\mathrm{Cu}$, and eye-guiding lines; most of the computations in the literature do not cover low- $Z$ systems) are shown. Note how at low $Z$ the lifetime predictions diverge by up to an order of magnitude. The computation by Berrington et al. comes closest overall to the low- $Z$ measurements-except for the measurement on Ar II by Lauer et al. [244] (the error bar is smaller than the symbol size) that Berrington did not know of at the time. Saha and Fritzsche, however, matched that one data point well by their computation, but they calculated only this one. The computations by Wilson seemed better than most others at the time-but covered too few elements. Mohan did well in 1992—but why were only two elements computed?

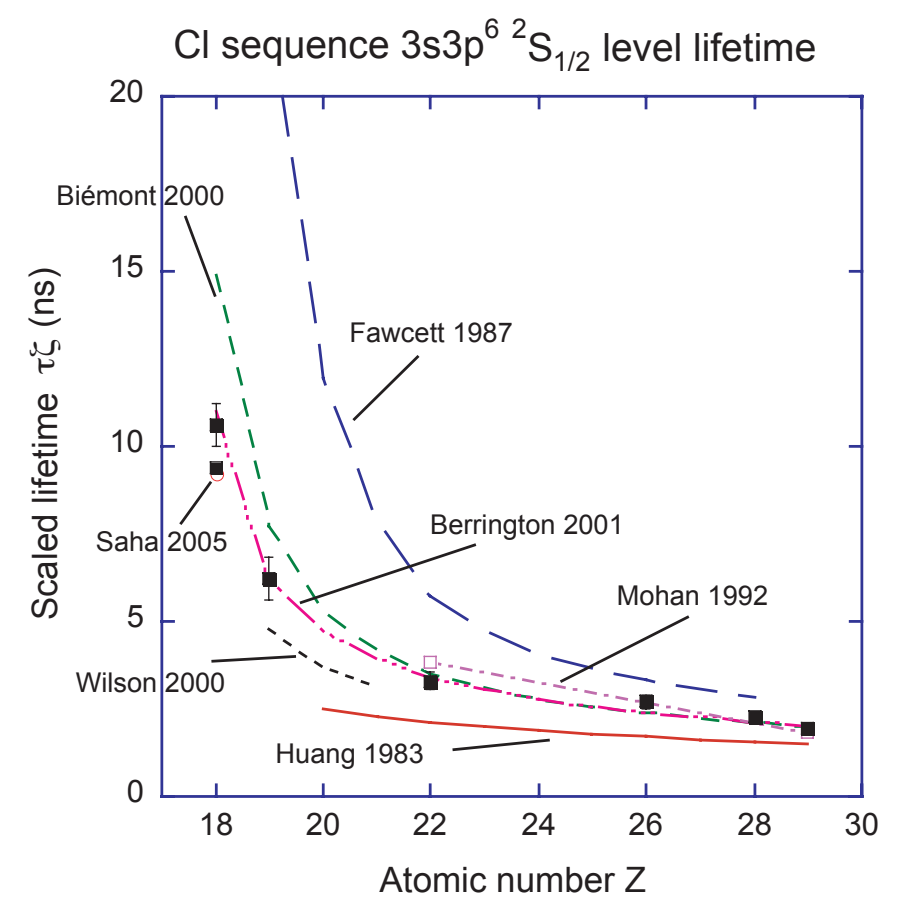

Figure 12 shows the state of affairs of experiment and theory concerning the $3 \mathrm{~s} 3 \mathrm{p}^{6}{ }^{2} \mathrm{~S}_{1 / 2}$ level lifetime in $\mathrm{Cl}$-like ions. While for moderate charge states the computations merge with the experimental data trend, the various computations diverge badly at low $Z$. Near-neutral ions are more difficult to calculate, but possibly more easily measured. However, the available lifetime data for Ar II differ by much more than their error bars from each other and by large factors from computations available at the time (see plots in a study geared towards low charge states [235]). A single (beam-foil) lifetime value exists also for Ti VI [241], which was followed 26 years later by a single-element computation [242]). Meanwhile a further beam-foil data point for $\mathrm{K}$ III has been added by H. P. Garnir at Liège ( $\tau=2.08 \pm 0.2 \mathrm{~ns}$ ) [243]. (Unfortunately, the lead experimenter has deceased since.) Most importantly, in a synchrotron experiment Ar gas has been ionized and excited, and the level lifetime in Ar II has been obtained with much improved accuracy [244]. While new computations [245,246] have claimed improved agreement with the beam-foil data, they did not include this most significant data point. Before too long, theory has corroborated this data point, too, but, alas, the successful computation [247] was done for this one ion only. I am repeating myself: What predictive value is in a computation that 
follows experiment and covers only the cases that experiment has already treated (here a single ion species)? It is good to know that an advanced atomic structure package has been used and that it has found a result in agreement with a measurement that had not been reproduced by computations before. But why (yes, it costs manpower and money) was the study not extended to a sequence of ions in order to yield an isoelectronic trend and predictions for other ions for which experiment had not yet told a result?

\subsection{Ar-Like Ions}

The Ar-like spectra do not figure as prominently in tabulations as the other sequences that have been discussed so far, although they are of interest for the interpretation of the cooler parts of the corona [248,249] and have repeatedly been modeled for line intensities (see work by Helen Mason). Spectral modeling usually proceeds with not the most complex type of computation, because one needs to identify candidate lines with peculiar properties under ranges of specific environmental conditions, usually being measured without extreme spectral resolution, other objectives ranking higher. However, there are many lines to consider, which consequently are individually weaker and thus more difficult to measure. Of the three resonance lines of Fe IX, $3 \mathrm{p}^{6}{ }^{1} \mathrm{~S}_{0}-3 \mathrm{p}^{5} 3 \mathrm{~d}{ }^{1} \mathrm{P}_{1}^{\mathrm{o}},{ }^{3} \mathrm{P}_{1}^{\mathrm{o}}$, and ${ }^{3} \mathrm{D}_{1}^{\mathrm{o}}$, with very different upper level lifetimes, only two have been seen so far apparently. The strongest of the three resonance lines in Fe IX and Ni XI can be seen in beam-foil spectra [212,250]. The Ar-like spectra appear at relatively low ion beam energies at which the thin carbon foils do not last very long under heavy-ion bombardment, posing a very practical limit to data accumulation. On the other hand, work at the Livermore EBIT has yielded lifetime information on 3d levels in Ar-like ions of $\mathrm{Kr}(\mathrm{Kr} \mathrm{XIX})$ that have major M2 decay branches [251]. Ar-like spectra are rich in lines, but only very partially known. For the present context, they are still too complex and have not yet been treated well enough.

We now leave the discursive sequence of isoelectronic sequences and jump to cases with many more electrons in total, but with only a few electrons outside of closed shells.

\subsection{Cu-Like Ions}

The third isoelectronic sequence treated by Kim et al. [100] and by Blundell [101] after Li I and Na I is not $\mathrm{KI}$ or Cs I (each with a single electron outside a rare-gas core configuration), but $\mathrm{Cu}$, with a single electron outside of a closed $3 d^{10}$ shell (Ni I sequence). At the Livermore EBIT, the $4 s_{1 / 2}-4 p_{3 / 2}$ resonance line has been followed along the isoelectronic sequence up to $Z=92$, and the data [103,252] have been compared to the veteran computations. For most of the way, the computations are as close to each other as to experiment, but at the highest values of $\mathrm{Z}$, the description by Blundell has worked out better. It is also noteworthy to realize that a fairly small EBIT device can provide $\mathrm{Cu}$-like ions of $\mathrm{U}$, whereas accelerators for beam-foil spectroscopy or lasers for laser-produced plasma work had to push their performance envelope to work in that range. Figure 3 of [103] also shows the systematic errors of the high- $Z$ non-EBIT data. If one looks for the computations associated with those other data, one can recognize that some of them had a bias in order to improve agreement with experimental data that by now can be seen as systematically flawed. This should serve as a reminder that ultimately computations with "adjustments" are to be avoided, because they may become misleading. 
For the resonance transitions in the $\mathrm{Cu}$ I sequence, the deviation between experiment and ab initio computation has become smaller than $200 \mathrm{ppm}$.

\subsection{Zn-Like Ions}

The same experiments that have yielded excellent agreement of measured and calculated $\mathrm{Cu}$-like ion resonance line energies provide data also on Zn-like ions, but the typical energy deviation has been on the order of 6000 to 8000 ppm, or 40 times more than for the $\mathrm{Cu}$ I sequence. Based on the very same spectra and the same calibrations, this wider gap has to be blamed on computations, of which there have been many (see [103,252], plus recent work at Notre Dame, Puerto Rico, or Livermore). Maybe the demonstration of the computational shortcomings has helped to renew interest in such problems on the theory side, and to new attempts and significant progress. From my perspective (possible memory lapses nonwithstanding) the story unfolded about as this:

In 2005, Vilkas and Ishikawa [253] employed their MR-MP second-order perturbation theory algorithms (plus QED corrections) to calculate the structure of high- $Z$ Zn-like ions. Their technique was based on Gaussian basis sets [254,255]. The deviations between the results of these atomic structure computations and a number of experiments shrank by one order of magnitude to a band of \pm 1000 ppm; most Livermore EBIT results and the computations differ by less than 500 ppm. In 2008, Blundell et al. [256] published MBPT results that came closer to experiment than most earlier computations, with the exception of Vilkas and Ishikawa's. The deviation was about $500 \mathrm{ppm}$ for W $(Z=74)$ and increased to about $1000 \mathrm{pm}$ for $\mathrm{U}(Z=92)$. In 2009, Blundell re-assessed the QED contributions and improved the agreement with experiment to better than 100 ppm [257]. In 2010, Safronova and Safronova, Blundell's coauthors of two years before, presented computations for more ions [258]; where the ion species were the same ones as before, the numbers remained unchanged. Their results and the isoelectronic trend pointed clearly away from the experimental EBIT data, but they matched a set of experimental results from laser-produced plasma work with their larger uncertainties and a systematic error trend (possibly related to the Doppler shift problem in observing a rapidly expanding plasma.). In the same journal issue, Chen and Cheng [259] have presented the results of their RCI computations which (with the exemptions of $\mathrm{Os}(Z=76)$ and $\mathrm{Pb}(Z=82))$ agree with all Livermore EBIT data, even slightly better than Blundell's results. The Chen and Cheng results furthermore include elements for which no measurements have been performed yet. At Puerto Rico, the Gaussian basis set has been expanded; as a result, an oscillation of the MR-MP computational results for the resonance transition energy in $\mathrm{Zn}$-like ions around the trends of the Blundell and the Chen and Cheng computations has thus been significantly reduced. The improved results have been published in 2011 [260]; a new EBIT measurement (on $\mathrm{Pt}(Z=78)$ ) and independent computations for the same ion having been presented already in 2010 [261].

In short, several types of computations of the resonance transition energy in high- $Z \mathrm{Zn}$-like ions ( $70 \geq Z \leq 92$ ) have now been brought to the same quality as reached for $\mathrm{Cu}$-like ions decades earlier. Similarly good computations for the $Z$ range below are still needed, as well as experimental data on other transitions. 


\subsection{Ga-Like and Ge-Like Ions}

The same MR-MP computations for Pt [262] also cover Ga- and Ge-like ions, corresponding to the Al- and Si-like ion work discussed above. Resonance $\left(4 \mathrm{~s}-4 \mathrm{p}_{3 / 2}\right)$ and intercombination $\left(4 \mathrm{~s}-4 \mathrm{p}_{1 / 2}\right)$ lines, respectively, of ions with one, two, three, or four electrons in the valence shell usually lie in close proximity to each other. This makes it attractive to analyze them together, but it provides/implies a challenge to theory. For the experiment it makes no fundamental difference whether a line arises from a system with a few or many electrons, but for computations, the difference is decisive, and it is a very good test. The experience has regularly been sobering: Li-, Na-, $\mathrm{Cu}$-like spectra can be calculated with high accuracy, and each additional electron brings about a major degradation of the computational accuracy. The $\mathrm{Zn}$-sequence spectra have experienced a major improvement only very recently (as discussed above), but this progress has not reached all atomic structure codes on the market. In fact, the readily observable signature lines in the few-electron spectra are a useful tool to identify by eye and to measure the offset between computations and measurement for the more complex systems [34,192,195,196,263-265]. The easily identified line pattern unfortunately disappears along the isoelectronic sequence, because the fine structure intervals grow much faster $\left(\propto Z^{4}\right)$ than the gross structure $(\propto Z)$. However, the fine structure intervals of the doublet ground term and the lowest quartet term in the systems with three electrons in the valence shell are rather similar, and thus at least certain line pairs or line groups survive for visual recognition. Can we expect theory to treat the many-electron systems equally well in the near future? The individual codes scale differently in the computational demands for more electrons. RCI may do best, but at a high cost. MR-MP does pretty well, as do other MBPT codes if supplemented with a lot of care. There are other codes that may be sufficient for a given purpose.

The intercombination line pair in Ge-like ions is a topic in which experiment still asks theory for assistance. The two lines are not particularly bright, and the wave number difference (the energy difference of the $4 \mathrm{~s}^{2} 4 \mathrm{p}^{2}{ }^{3} \mathrm{P} J=1$ and $J=2$ levels) is not known from experiment. In beam-foil spectra, many lines fill the neighborhood, and the line identifications for some of the ions are tentative only. When trying to survey the data base, I have found actual entries of explicitly "estimated" quintet level positions, and by my nearby observations I could tell that the estimates were wrong. The spectra of ions with two, three, or four valence shell electrons appear to provide a useful test of performance quality. I hope that improved computations will become available for a re-analysis of existing spectra so that the frontiers of a reliable data set can be expanded.

\section{Pm-Like, Sm-Like, Eu-Like Ions}

And now a little recreational digression. A third of a century ago, Curtis and Ellis have come up with a neat idea. Although the neutral atom promethium $(\mathrm{Pm})$ does not have a single valence electron outside of closed shells only (in this case, filled up to $4 \mathrm{f}^{14}$ ), high charge state ions in the Pm isoelectronic sequence might feature a $5 \mathrm{~s}-5 \mathrm{p}$ pair of resonance line analogous to the alkali atoms [266]. Beam-foil spectra of $\mathrm{Au}$ recorded at Bochum [267] showed clusters of very many lines among which it was not possible to pick out the right ones with confidence, but it became obvious that the lines of interest were not as prominent as the resonance lines in the simple alkali spectra. A few years later, Bochum demonstrated that in the adjacent isoelectronic sequences ( $\mathrm{Sm}, \mathrm{Eu}, \mathrm{Gd})$ the intercombination transitions might be 
filtered out by the time-resolution inherent to beam-foil work, and among many weak lines, candidates were found with level lifetimes of the predicted magnitude [268]. More beam-foil work was tried at RIKEN in Japan, EBIT work (fewer lines) at Berlin, a tokamak observation on U thrown in, and the computations were upgraded. MR-MP computations by Vilkas and Ishikawa [269] indicated that, with the possible exception of the heaviest elements, the $5 \mathrm{~s}-5 \mathrm{p}$ line pair would not be easily recognizable- their upper and lower levels were embedded among hundreds of other levels, and no parameter made the lines stand out. While the partial selectivity of the excitation process in EBITs (the spectra are heavily weighted towards ground state transitions) renders most EBIT spectra much simpler than the extremely line-rich beam-foil spectra, this is not good enough to produce the $5 s-5 p$ lines prominently. The Berlin EBIT group practically ascertained that they could optimize their EUV spectra of tungsten for specific charge states, but they did not see lines in good agreement with the wavelength predictions for the $5 \mathrm{~s}-5 \mathrm{p}$ transitions. This should be no surprise, as has repeatedly been discussed (see [269]). In the latest twist of the story, a Japanese group has calculated that there is another reason, too, in that (at least for Pm-like ions of $\mathrm{Bi}(Z=83))$ there is a long-lived level that acts as a population trap and hinders the population of the signature levels [270].

If one cannot identify the lines in the observed spectra, it is difficult to judge the accuracy of the computations. EBIT spectra with their much fewer lines carry much less potential wavelength information, but what there is, can be mined with higher accuracy (no Doppler shift etc.). However, one has to model theoretical spectra for the excitation conditions in an EBIT. Typical modeling codes are based on HULLAC or FAC computations which are not as accurate in atomic structure terms as are dedicated large-scale atomic structure computations. With hundreds, if not thousands, of levels to consider, it seems highly desirable to develop an interface that feeds the output of accurate structure computations into the data set for the collisional-radiative modeling exercise. This is a tedious task, but it would be helpful for the study of many multi-electron atomic systems.

A much less tedious task, with a much shorter time line, would be to refrain from pretending that the resonance lines of any Pm-like ions are of any likely future use in the diagnostics of fusion reactors. Since decades it has been established that these lines are not prominent (if seen at all, which in most cases remains uncertain). The hot (Doppler broadening) multi-element environment of a fusion plasma won't make the observation and application any easier.

\section{W}

In the course of development work on controlled fusion, a long-term mantra has been that any material of a nuclear charge higher than $Z=3$ must be avoided, because it would cause unbearable radiative losses to the fledgling plasma and thus hinder fusion conditions from being reached. However, the vacuum vessel would likely be built of steel, and this fact motivated data collection on the spectra of Fe $(Z=26)$ and elements that would likely be used in special alloys, from $\mathrm{V}(Z=23)$ and $\mathrm{Cr}(Z=24)$ to Mo $(Z=42)$. This purpose is still recognizable in the abundance and distribution of data in prominent data bases. In order to keep contaminations out of the plasma, the vessel surfaces were then coated with light elements such as Be, B, or C. Nowadays the power density of the fusion test beds has become so high that the robustness of the vessel under heat load and neutron bombardment is a primary concern, 
and now tungsten (W, $Z=74$ ) as the most heat-resistant element is touted as the inevitable material for coating certain surfaces. Tungsten offers the additional advantage that it will not be fully ionized in the center of the hot plasma. Therefore spectral observations of $\mathrm{W}$ contaminations can be made in all locations of the plasma, and the spectra will tell about the temperature (by charge state distribution) and other plasma parameters without any need to inject additional contaminants. This set of ideas has initiated an avalanche of studies of the various spectra of $\mathrm{W}$ and of charge exchange $(\mathrm{CX})$ processes. NIST Gaithersburg has undertaken efforts (first by Joseph Reader and now by Alexander Kramida) to catalogue the available data [271] as well as pursuing some computations and measurements of their own (Yuri Ralchenko). The International Atomic Energy Agency (IAEA) has supported seminars at which people interested in studying W supposedly coordinate their efforts. In his time at the Livermore EBIT group, Joel Clementson has started a tungsten project ("WOLFRAM") with experiments at various plasma machines, and among other things he has demonstrated high-resolution spectra of the famous E2 and M3 lines in Ni-like W (first recognized years ago in EBIT spectra of Th and U [272]) which are discussed for ITER diagnostics [273-277]. Besides the observers/experimenters, theorists have risen to the challenges of multi-electron system atomic structure. For example, Marius Vilkas at UPR has used the MR-MP code to calculate levels and transition rates of W ions with charges 7+ (Ho-like) to 15+ (Pr-like), but the results were too rich to be published in print. Jon Grumer at Lund is working on the next higher charge states using MCDF codes. The Safronovas have addressed Ne-, Na-, Mg-, Cu-, Ag-, Pd-, Er-, Yb-, Y-, Pm-like spectra of W (and probably even more), and so on. (I am not going to add a catalog of my own.) There are spectroscopic details of some individual studies that ought to be taken up by data bases. However, besides some lighthouse features there are thousands of lines in measured spectra and computations, and nobody has the resources to actually combine the two reservoirs in detail. Although it would be good to extract the information once and for all and have it available for easy retrieval, this is not likely to happen. The fusion plasma community most likely will need collisional-radiative modeling of some quality and fidelity to accurate data, but without the explicit atomic structure background detail. This may be intellectually disappointing to spectroscopists who try their best to extract the detail, but it reflects the limitations of funding. Tungsten spectra have very many lines, but there will not be enough funding available, and certainly not in proportion to the number of spectral lines seen or calculated.

\section{Discussion}

The task put to me was to discuss how an experimenter judges computations and their results. Theory (computation) and experiment, however, are entangled entities of atomic physics which one cannot readily separate. Beam-foil spectroscopy has thrived, because the ion-foil interaction effectively populates all levels. Unfortunately, the spectra of the excited ions then often are so full of lines (many from the decays of core-excited levels) that any attempt at analysis is overburdened. The technique works beautifully on few-electron ions and on transitions with outstandingly bright lines or with decays slow enough compared to others that (easily achieved) time discrimination helps. A bane of beam-foil spectroscopy is the need for Doppler shift correction of observed wavelengths, which would be relatively easy if enough reference lines were already known. Laser-produced plasmas also suffer from Doppler shifts (in an expanding plasma) which have tainted some measurements that were hoped to determine 
wavelength standards. Electron beam ion traps have no Doppler shift problem, since the trapped ion cloud is rather stationary. However, the light source operates as a low-density environment in which most ions will be in their ground state or metastable states, and the spectra are dominated by ground state transitions, in a stark contrast to beam-foil spectroscopy (where a high total angular momentum lends a large statistical weight and thus offers plenty of phase space to population processes). Fewer lines in the spectrum avoid many problems of line blends and position determination, but one often needs collisional-radiative modeling computations to ascertain which lines should be seen and which ones should not appear. The difference can be drastic (see and compare [34,192,197,278]). The difference also appears in astrophysical data. For example, the NIST online data base ASD [279] carries the legacy of line intensities obtained by whatever measurement or estimate over the decades, from the days of visual estimates to modern photoelectric detectors. In contrast, the CHIANTI data base [280,281] aims at providing accurate wavelength data of all elements and charge states of interest in the solar corona, but the line intensity data are derived from modelling for specific representative electron densities and temperatures. This wavelength data base combines observations and scaled computations, as the user has to be aware of.

Highly charged heavy ions are much more easily produced and excited in an EBIT (which fits into a moderate-size laboratory) than in a heavy-ion accelerator that has to accelerate ions to so much higher energies than the electron beam in an EBIT_-because of the electron-nucleon mass ratio-for reaching the same relative velocity between projectile and target. There are various active collaborations trying to combine experimental data and computational results on the same atomic systems. Such co-operative efforts can be very fruitful, providing a link between observations of nature and theoretical concepts, between atomic structure computations and reality. The toolboxes on either side are not yet exhausted. A major tool, long time in the making, is the selective excitation of multiply charged ions. Some two decades ago the Oxford EBIT group has started a project on laser excitation. Meanwhile the Heidelberg EBIT group has laser-excited E1-forbidden transitions of trapped highly charged ions [282] and has moved an EBIT to the FLASH facility at Hamburg to effect selective excitation of highly charged ions by EUV light from a free electron laser [117]. As already mentioned, this line of work has then been extended to excitation by X-ray light at the LCLS facility at Stanford [187], with results in a clear discordance with theoretical expectation. Much more is possible. Experimenters just like to test (and challenge) theoretical computations of atomic structure and transition probabilities by accurate measurements!

\section{Acknowledgments}

Over the years, the German research council DFG has been very supportive to my work. I have worked in various places, most extensively at the Bochum accelerator laboratory and with the Livermore EBIT group, and I have benefited greatly also from further experimental collaborations and from most helpful theoreticians. Since this report is (partly) an opinion piece, I refrain from naming any colleagues in particular - they are practically all cited below, with some part of other of their work. In a citation list at least twice as long, I might have come somewhat closer to a fair representation of all their contributions to my present topic. 


\section{Conflicts of Interest}

The author declares no conflict of interest.

\section{References}

1. Curtis, L.J.; Martinson, I. Recent Progress in Atomic-Structure Investigations with Fast-Ion Beams. Comments Atom. Molec. Phys. 1980, 10, 1-8.

2. Martinson, I.; Ellis, D.G. Recent studies of intercombiantion lines in ions. Comments Atom. Mol. Phys. 1985, 16, 21-33.

3. Ellis, D.G.; Martinson, I.; Träbert, E. Intercombination transitions in multiply ionized atoms. Comments Atom. Mol. Phys. 1989, 22, 241-262.

4. Curtis, L.J.; Martinson, I. Lifetime Measurements in Multiply Ionized Atoms. Comments Atom. Mol. Phys. 1990, 24, 213-233.

5. Curtis, L.J. Precision Oscillator Strength and Lifetime Measurements. In Atomic, Molecular, and Optical Physics Reference Book; Drake, G.W.F., Ed.; AIP Press: New York, NY, USA, 1996; pp. 206-212.

6. Träbert, E. Atomic lifetime measurements with ion traps of many sizes. Phys. Scr. 2000, 61, 257-286.

7. Träbert, E. Lifetime measurements of highly charged ions. Phys. Scr. T 2002, 100, 88-97.

8. Träbert, E. Precise measurements of long atomic lifetimes using stored ion beams and ion traps. Can. J. Phys. 2002, 80, 1481-1503.

9. Mannervik, S. Experimental lifetime studies of metastable levels. Phys. Scr. T 2003, 105, 67-75.

10. Träbert, E. Atomic lifetime measurements using electron beam ion traps. Can. J. Phys. 2008, 86, 73-97.

11. Träbert, E. Problems with accurate atomic lifetime measurements of multiply charged ions. Phys. Scr. 2009, 79, 068101.

12. Träbert, E. In pursuit of high precision atomic lifetime measurements of multiply charged ions. J. Phys. B: At. Mol. Opt. Phys. 2010, 43, 074034.

13. Träbert, E. Radiative-lifetime measurements on highly-charged ions. In Accelerator-based Atomic Physics Techniques and Applications; Shafroth, S.M., Austin, J.C., Eds.; American Institute of Physics: Washington, DC, USA, 1997; pp. 567-607

14. Curtis, L.J. A diagrammatic mnemonic for computation of cascading level populations. Am. J. Phys. 1968, 36, 1123-1128

15. Curtis. L.J.; Berry, H.G.; Bromander, J. A meanlife measurement of the $3 \mathrm{~d}^{2} \mathrm{D}$ resonance doublet in Si II by a technique which exactly accounts for cascading. Phys. Lett. A 1971, 34, 169-170.

16. Engström, L. CANDY, a computer program to perform an ANDC analysis of cascade correlated decay curves. Nucl. Instrum. Meth. 1982, 202, 369-376.

17. Engström, L. Studies of atomic decay properties using fast ion beam spectroscopy. Phys. Scr. 1989, 40, 17-24.

18. Träbert, E.; Heckmann, P.H. Beam-foil lifetimes of Be-like ions of elements from $\mathrm{Mg}$ to S. Phys. Scr. 1980, 22, 489-492. 
19. Träbert, E.; Johnson, B.M.; Jones, K.W.; Gregory, D.C.; Kruse, T.H. Beam-foil lifetimes of $n=3$ levels in Fe XIII to Fe XVI. II. Analysis of decay curves. Phys. Lett. A 1982, 87, 336-340.

20. Träbert, E.; Doerfert, J.; Granzow, J.; Büttner, R.; Brauckhoff, J.; Nicolai, M.; Schartner, K.-H.; Folkmann, F; Mokler, P.H. Lifetimes of $n=2$ levels in foil-excited $\mathrm{Kr}^{32+}$ and $\mathrm{Kr}^{33+}$ ions. Phys. Lett. A 1995, 202, 91-98.

21. Träbert, E.; Curtis, L.J. Isoelectronic trends of line strength data in the Li and Be isoelectronic sequences. Phys. Scr. 2006, 74, C42-C50.

22. Reistad, N.; Martinson, I. Accurate transition probabilities in ions obtained by isoelectronic smoothing of line strengths. Phys. Rev. A 1986, 34, 2632-2637.

23. Träbert, E. Isoelectronic smoothing of oscillator strengths in the beryllium sequence. Z. Phys. $D$ 1988, 9, 143-145.

24. Curtis, L.J. Isoelectronic smoothing of line strengths in intermediate coupling. Phys. Scr. 1991, 43, 137-143.

25. Hylleraas, E.A. Neue Berechnung der Energie des Heliums im Grundzustande, sowie des tiefsten Terms von Ortho-Helium. Z. Phys. 1929, 54, 347-366.

26. Hylleraas, E.A. Über den Grundterm der Zweielektronenprobleme von $\mathrm{H}^{-}, \mathrm{He}, \mathrm{Li}^{+}, \mathrm{Be}^{++}$usw. Z. Phys. D 1930, 65, 209-225.

27. Jönsson, P.; Froese Fischer, C.; Träbert, E. On the status and perspectives of MCDF computations and measurements of transition data in the Be isoelectronic sequence. J. Phys. B: At. Mol. Opt. Phys. 1998, 31, 3497-3511.

28. Froese Fischer, C. The MCHF/MCDHF collection. 2004 Available online: nlte.nist.gov/MCHF/ (accessed on 15 Nov 2013).

29. Curtis, L.J.; Ellis, D.G. Predictive systematization of line strengths for the $2 s^{2}-2 s 2 p$ resonance and intercombination transitions in the Be isoelectronic sequence. J. Phys. B: At. Mol. Opt. Phys. 1996, 29, 645-654.

30. Martinson, I.; Gaupp, A.; Curtis, L.J. Comments on the Be I $2 \mathrm{~s}^{2}{ }^{1} \mathrm{~S}-2 \mathrm{~s} 2 \mathrm{p}{ }^{1} \mathrm{P}$ transition probability. J. Phys. B: At. Mol. Phys. 1974, 7, L463-L466.

31. Irving, R.E.; Henderson, M.; Curtis, L.J.; Martinson, I.; Bengtsson, P. Accurate transition probabilities for the $2 \mathrm{~s}^{2}{ }^{1} S-2 \mathrm{~s} 2 \mathrm{p}{ }^{1} \mathrm{P}$ transition in Be I and B II. Can. J. Phys. 1999, 77, 137-143.

32. Träbert, E.; Pinnington, E.H.; Kernahan, J.A.; Doerfert, J.; Granzow, J.; Heckmann, P.H.; Hutton, R. Beam-foil study of the lifetimes of $n=3$ levels in Na-like Ca X, Mg-like Ca IX and Si-like Ca VII. J. Phys. B: At. Mol. Opt. Phys. 1996, 29, 2647-2659.

33. Hutton, R.; Engström, L.; Träbert, E. Observation of a discrepancy between experimentally determined atomic lifetimes and relativistic predictions for highly ionized members of the $\mathrm{Na}$ I isoelectronic sequence. Phys. Rev. Lett. 1988, 60, 2469-2472.

34. Träbert, E.; Doerfert, J.; Granzow, J.; Büttner, R.; Staude, U.; Schartner, K.H.; Rymuza, P.; Mokler, P.H.; Engström, L.; Hutton, R. Experimental $n=3$ level lifetimes of Na-like heavy ions in the realm of massive relativistic effects. Phys. Lett. A 1994, 188, 355-360.

35. Kink, I.; Hutton, R.; Nyström, B.; Martinson, I.; Ishii, K.; Ando, K.; Kambara, T.; Nakai, Y.; Kojima, T.M.; Awaya, Y. Lifetime of the $3 \mathrm{p}^{2} \mathrm{P}_{3 / 2}$ level in Na-like $\mathrm{Kr}^{25+}$. Phys. Rev. A 1997, 55, 3229-3232. 
36. Vasilyev, A.; Jasper, E.; Berry, H.G.; Livingston, A.E.; Curtis, L.J.; Cheng, S.; Dunford, R.W. Lifetime of the $3 \mathrm{p}^{2} \mathrm{P}_{3 / 2}$ level in sodiumlike bromine (Br XXV). Phys. Rev. A 1998, 58, 732-735.

37. Schnabel, R.; Kock, M. f-value measurement of the Be I resonance line using a nonlinear time-resolved laser-induced-fluorescence technique. Phys. Rev. A 2000, 61, 062506.

38. Verdebout, S.; Rynkun, P.; Jönsson, P.; Gaigalas, G.; Froese Fischer, C.; Godefroid, M. A partitioned correlation function interaction approach for describing electron correlation in atoms. J. Phys. B: At. Mol. Opt. Phys. 2013, 46, 085003.

39. Wiese, W.L.; Smith, M.W.; Glennon, B.M. NSRDS-NBS4-Atomic Transition Probabilities; Government Printing Office: Washington, DC, USA, 1966; Volume I Hydrogen Through Neon, pp. 1-169.

40. Kwong, H.S.; Smith, P.L.; Parkinson, W.H.; Knight, R.D. Transition probability of the C III $190.9 \mathrm{~nm}$ intersystem line. Bull. Am. Phys. Soc.1983, 28, 781.

41. Smith, P.L.; Kwong, H.S.; Johnson, B.C.; Parkinson, W.H. Measurements of A-values for C III $(1909 \AA)$ and O III $(1660,1666 \AA)$ intersystem lines used in solar transition zone electron density determinations. Bull. Am. Astron. Soc. 1983, 15, 703.

42. Smith, P.L.; Johnson, B.C.; Kwong, H.S.; Parkinson, W.H.; Knight, R.D. Measurements of transition probabilities for spin-changing lines of atomic ions used in diagnostics of astrophysical plasmas. Phys. Scr. T 1984, 8, 88-94.

43. Kwong, V.H.S.; Fang, Z.; Gibbons, T.T.; Parkinson, W.H.; Smith, P.L. Measurement of the transition probability of the C III 190.9 nanometer intersystem line. Astrophys. J. 1993, 411, 431-437.

44. Wiese, W.L.; Fuhr, J.R.; Deters, T.M. Atomic Transition Probabilities of Carbon, Nitrogen, and Oxygen: A Critical Data Compilation, Series: Journal of Physical and Chemical Reference Data, Monograph, No. 7; American Chemical Society: Washington, DC, USA, 1996; pp. 1-522.

45. Chou, H.-S.; Chi, H.-S.; Huang, K.-N. Core polarization effects on the intercombination and resonance transitions in Be-like ions. Chin. J. Phys. 1994, 32, 261-268.

46. Ral'chenko, Y.V.; Vainshtein, L.A. Intercombination transitions in Be-like ions. Phys. Rev. A 1995, 52, 2449-2452.

47. Fleming, J.; Hibbert, A.; Stafford, R.P. The 1909 A intercombination line in C III. Phys. Scr. 1994, 49, 316-322.

48. Froese Fischer, C. Allowed transitions and intercombination lines in C III and C II. Phys. Scr. 1994, 49, 323-330.

49. Ynnerman, A.; Froese Fischer, C. Multiconfigurational-Dirac-Fock computation of the $2 \mathrm{~s}^{2}{ }^{1} \mathrm{~S}_{0}-2 \mathrm{~s} 2 \mathrm{p}{ }^{3} \mathrm{P}_{1}$ spin-forbidden transition for the Be-like isoelectronic sequence. Phys. Rev. A 1995, 51, 2020-2030.

50. Doerfert, J.; Träbert, E.; Wolf, A.; Schwalm, D.; Uwira, O. Precision measurement of the electric dipole intercombination rate in $\mathrm{C}^{2+}$. Phys. Rev. Lett. 1997, 78, 4355-4358.

51. Curtis, L.J. Private Communication.

52. Chen, M.H.; Cheng, K.T.; Johnson, W.R. Large-scale relativistic configuration-interaction computation of the $2 \mathrm{~s}^{2}{ }^{1} \mathrm{~S}_{0}-2 \mathrm{~s} 2 \mathrm{p}{ }^{3} \mathrm{P}_{1}$ intercombination transition in C III. Phys. Rev. A 2001, 64, 042507. 
53. Kelly, R.L.; Palumbo, L.J. Atomic and Ionic Emission Lines Below $2000 \AA$ A, Hydrogen through Krypton; Naval Research Laboratory: Washington, DC, USA, 1973; pp. 1-1001.

54. Vilkas, M.J.; Ishikawa, Y.; Träbert, E. Relativistic many-body perturbation computations of boron-like silicon, Si X. Phys. Scr. 2005, 72, 181-199.

55. Andrä, H.J.; Gaupp, A.; Wittmann, W. New method for precision lifetime measurements by laser excitation of fast-moving atoms. Phys. Rev. Lett. 1973, 31, 501-504 .

56. Gaupp, A.; Kuske, P.; Andrä, H.J. Accurate lifetime measurements of the lowest ${ }^{2} \mathrm{P}_{1 / 2}$ states in neutral lithium and sodium. Phys. Rev. A 1982, 26, 3351-3359.

57. Schulze-Hagenest, D. Ph.D. Thesis, University of Kaiserslautern, Kaiserslautern, Germany, 1979; cited in [56].

58. Carlsson, J.; Sturesson, L. Accurate time-resolved laser spectroscopy on lithium atoms. Z. Phys. D 1989, 14, 281-287.

59. Carlsson, J. Laser spectroscopic studies of lifetimes in neutral atoms. Phys. Scr. 1989, 39, 442-446.

60. Carlsson, J.; Jönsson, P.; Sturesson, L.; Froese Fischer, C. Multi-configuration Hartree-Fock calculations and time-resolved laser spectroscopic studies of hyperfine constants in sodium. Phys. Scr. 1992, 46, 394-398.

61. McAlexander, W.I.; Abraham, E.R.I.; Ritchie, N.W.M.; Williams, C.J.; Stoof, H.T.C.; Hulet, R.G. Precise atomic radiative lifetime via photoassociative spectroscopy of ultracold lithium. Phys. Rev. A 1995, 51, R871-R874.

62. McAlexander, W.I., Abraham, E.R. I.; Hulet, R.G. Radiative lifetime of the ${ }^{2} \mathrm{P}$ state of lithium. Phys. Rev. A 1996, 54, R5-R8.

63. Volz, U.; Majerus, M.; Liebel, H.; Schmitt, A.; Schmoranzer, H. Precision lifetime measurements on Na I $3 p^{2} \mathrm{P}_{1 / 2}$ and $3 p^{2} \mathrm{P}_{3 / 2}$ by beam-gas-laser spectroscopy. Phys. REv. Lett. 1996, 76, 2862-2865.

64. Astner, G.; Curtis, L.J.; Liljeby, L.; Mannervik, S.; Martinson, I. A high precision beam-foil meanlife measurement of the $1 \mathrm{~s} 3 \mathrm{p}{ }^{1} \mathrm{P}$ level in He I. Z. Phys. A 1976, 279, 1-6.

65. Martinson, I. Private Communication.

66. Cheng, K.T.; Kim, Y.-K.; Desclaux, J.P. Electric dipole, quadrupole, and magnetic dipole transition probabilities of ions isoelectronic to the first-row atoms, Li through F. At. Data Nucl. Data Tables 1979, 24, 111-189.

67. Huang, K.-N. Energy-level scheme and transition probabilities of Al-like ions. At. Data Nucl. Data Tables 1986, 34, 1-77.

68. Huang, K.-N. Energy-level scheme and transition probabilities of Si-like ions. At. Data Nucl. Data Tables 1985, 32, 503-566.

69. Huang, K.-N. Energy-level scheme and transition probabilities of P-like ions. At. Data Nucl. Data Tables 1984, 30, 313-421.

70. Huang, K.-N.; Kim, Y.-K.; Cheng, K.T.; Desclaux, J.P. Energy-level scheme and transition probabilities of Cl-like ions. At. Data Nucl. Data Tables 1983, 28, 355-377.

71. Huang, K.-N.; Johnson, W.R. Resonance transitions of Mg-and Zn-like ions from multiconfiguration relativistic random-phase approximation. Nucl. Instrum. Meth. Phys. Res. B 1985, 9, 502-504. 
72. Chou, H.-S.; Chi, H.-C.; Huang, K.-N. Relativistic excitation energies and oscillator strengths including core-polarization effects for the intercombination and resonance transitions in $\mathrm{Mg}$-like ions. J. Phys. B: At. Mol. Opt. Phys. 1993, 26, 4079-4089.

73. Johnson, W.R.; Soff, B. The Lamb shift in hydrogen-like atoms, $1 \leq Z \leq 110$. At. Data Nucl. Data Tables 1985, 33, 405-446.

74. Beiersdorfer, P.; Osterheld, A.; Scofield, J.; Crespo López-Urrutia, J.; Widmann, K. Measurement of QED and hyperfine splitting in the $2 \mathrm{~s}_{1 / 2}-2 \mathrm{p}_{3 / 2}$ X-ray transition in Li-like ${ }^{209} \mathrm{Bi}^{80+}$. Phys. Rev. Lett. 1998, 80, 3022-3025.

75. Beiersdorfer, P.; Knapp, D.; Marrs, R.E.; Elliott, S.R.; Chen, M.H. Structure and Lamb shift of $2 \mathrm{~s}_{1 / 2}-2 \mathrm{p}_{3 / 2}$ levels in lithiumlike $\mathrm{U}^{89+}$ through neonlike $\mathrm{U}^{82+}$. Phys. Rev. Lett. 1993, 71, 3939-3942.

76. Beiersdorfer, P.; Chen, H.; Thorn, D.B.; Träbert, E. Measurement of the two-loop Lamb shift in lithiumlike $\mathrm{U}^{89+}$. Phys. Rev. Lett. 2005, 95, 233003.

77. Kozhedub, Y.S.; Andreev, O.V.; Shabaev, V.M.; Tupitsyn, I.I.; Brandau, C.; Kozhuharov, C.; Plunien, G.; Stöhlker, T. Nuclear deformation effect on the binding energies in heavy ions. Phys. Rev. A 2008, 77, 032501.

78. Shabaev, V.M.; Andreev, O.V.; Bondarev, A.I.; Glazov, D.A.; Kozhedub, Y.S.; Maiorova, A.V.; Plunien, G.; Tupitsyn, I.I.; Volotka, A.V. Quantum electrodynamics effects in heavy ions and atoms. AIP Conf. Proc. 2011, 1344, 60-69.

79. Johnson, W.R.; Plante, D.R.; Sapirstein, J. Relativistic Computations of Transition Amplitudes in the Helium Isoelectronic Sequence. In Advances of Atomic, Molecular, and Optical Physics; Bederson, B., Walther, H., Eds.; Academic Press: San Diego, CA, USA, 1995; Volume 35, pp. 255-329.

80. Toleikis, S.; Manil, B.; Berdermann, E.; Beyer, H.F.; Bosch, F.; Czanta, M.; Dunford, R.W.; Gumberidze, A.; Indelicato, P.; Kozhuharov, C.; et al. Lifetime of the $2{ }^{3} \mathrm{P}_{0}$ state of He-like ${ }^{197} \mathrm{Au}$. Phys. Rev. A 2004, 69, 022507.

81. Munger, C.T.; Gould, H. Lamb shift in heliumlike uranium $\left(\mathrm{U}^{90+}\right)$. Phys. Rev. Lett. 1986, 57, 2927-2930.

82. Reuschl, R; Gassner, T.; Spillmann, U.; Bräuning-Demian, A.; Ananyeva, A.; Beyer, H.; Blumenhagen, K.-H.; Chen,W.; Hagmann, S.; Hegewald, M.; et al. Lifetime measurement of the $2{ }^{3} \mathrm{P}_{0}$ state in He-like uranium. Phys. Scr. T 2013, 156, 014024.

83. Trassinelli, M.; Kumar, A.; Beyer, H.F.; Indelicato, P.; Märtin, R.; Reuschl, R.; Kozhedub, Y.S.; Brandau, C.; Bräuning, H.; Geyer, S.; et al. Observation of the $2 \mathrm{p}_{3 / 2} \rightarrow 2 \mathrm{~s}_{1 / 2}$ intra-shell transition in He-like uranium. Europhys. Lett. 2009, 87, 63001.

84. Gabriel, A.H.; Jordan, C. Long wavelength satellites to the He-like ion resonance lines in the laboratory and the sun. Nature 1969, 221, 947-949.

85. Drake, G.W.F. Theory of relativistic magnetic dipole transitions: Lifetime of the metastable $2{ }^{3} \mathrm{~S}$ state of the heliumlike ions. Phys. Rev. A 1971, 3, 908-915.

86. Lin, C.D. Ph.D. Thesis. Columbia University, New York, NY, USA, 1975. 
87. Schmidt, H.T.; Forck, P.; Grieser, M.; Habs, D.; Kenntner, J.; Miersch, G.; Repnow, R.; Schramm, U.; Schüssler, T.; Schwalm, D.; et al. High-precision measurement of the magnetic-dipole decay rate of metastable heliumlike carbon ions in a storage ring. Phys. Rev. Lett. 1994, 72, 1616-1619.

88. Saghiri, A.A.; Linkemann, J.; Schmitt, M.; Schwalm, D.; Wolf, A.; Bartsch, T.; Hoffknecht, A.; Müller, A.; Graham, W.G.; Price, A.D.; et al. Dielectronic recombination of ground-state and metastable $\mathrm{Li}^{+}$ions. Phys. Rev. A 1999, 60, R3350-R3353.

89. Träbert, E.; Gwinner, G.; Knystautas, E.J.; Wolf, A. Heavy-ion storage-ring quest for atomic lifetimes in $\mathrm{Li}^{+}$and $\mathrm{Be}^{2+}$. Can. J. Phys. 2003, 81, 941-952.

90. Crespo López-Urrutia, J.R.; Beiersdorfer, P.; Savin, D.W.; Widmann, K. Precision measurement of the lifetime of the $1 \mathrm{~s} 2 \mathrm{~s}^{3} \mathrm{~S}_{1}$ metastable level in heliumlike $\mathrm{O}^{6+}$. Phys. Rev. A 1998, 58, 238-241.

91. Träbert, E.; Beiersdorfer, P.; Brown, G.V.; Smith, A.J.; Utter, S.B.; Gu, M.F.; Savin, D.W. Improved electron-beam ion-trap lifetime measurement of the $1 \mathrm{~s} 2 \mathrm{~s}{ }^{3} \mathrm{~S}_{1}$ level in $\mathrm{Ne}^{8+}$. Phys. Rev. A 1999, 60, 2034-2038.

92. Crespo López-Urrutia, J.R.; Beiersdorfer, P.; Widmann, K. Lifetime of the $1 \mathrm{~s} 2 \mathrm{~s}{ }^{3} \mathrm{~S}_{1}$ metastable level in He-like $\mathrm{S}^{14+}$ measured with an electron beam ion trap. Phys. Rev. A 2006, 74, 012507.

93. Dunford, R.W.; Church, D.A.; Liu, C.J.; Berry, H.G.; Raphaelian, M.L.A.; Hass, M.; Curtis, L.J. Lifetime of the $2{ }^{3} \mathrm{~S}_{1}$ state of heliumlike ${ }^{79} \mathrm{Br}^{33+}$. Phys. Rev. A 1990, 41, 4109-4111.

94. Birkett, B.B.; Briand, J.-P.; Charles, P.; Dietrich, D.D.; Finlayson, K.; Indelicato, P.; Liesen, D.; Simionovici, A. Hyperfine quenching and measurement of the $2{ }^{3} \mathrm{P}_{0}-2{ }^{3} \mathrm{P}_{1}$ fine-structure splitting in heliumlike silver $\left(\mathrm{Ag}^{45+}\right)$. Phys. Rev. A 1993, 47, R2454-R2457.

95. Simionovici, A.; Birkett, B.B.; Marrus, R.; Charles, P.; Indelicato, P.; Dietrich, D.D.; Finlayson, K. Cascade-free Doppler-tuned precision measurement of the $2{ }^{3} \mathrm{~S}_{1}$ state in He-like niobium $\left({ }^{93} \mathrm{Nb}^{39+}\right)$. Phys. Rev. A 1994, 49, 3553-3556.

96. Marrus, R.; Charles, P.; Indelicato, P.; de Billy, L.; Tazi, C.; Briand, J.-P.; Simionovici, A.; Dietrich, D.D.; Bosch, F.; Liesen, D. Lifetime of the $2{ }^{3} \mathrm{~S}_{1}$ state of heliumlike xenon $\left(\mathrm{Xe}^{52+}\right)$. Phys. Rev. A 1989, 39, 3725-3727.

97. Hubricht, G.G.; Träbert, E. The argon $2{ }^{3} \mathrm{~S}_{1}$ lifetime from a measurement on recoil ions. Z. Phys. $D$ 1987, 7, 243-250.

98. Moos, H.W.; Woodworth, J.R. Observation of the forbidden $2{ }^{3} S_{1} \rightarrow 1{ }^{1} S_{0}$ spontaneous emission line from helium and measurement of the transition rate. Phys. Rev. Lett. 1973, 30, 775-778.

99. Saathoff, G.; Reinhardt, S.; Karpuk, S.; Träbert, E. Quest for higher precision on the Li II M1 transition rate. 2004, unpublished.

100. Kim, Y.-K.; Baik, D.H.; Indelicato, P.; Desclaux, J.P. Resonance transition energies of Li-, Na-, and $\mathrm{Cu}$-like ions. Phys. Rev. A 1991, 44, 148-166.

101. Blundell, S.A. computations of the screened self-energy and vacuum polarization in Li-like, Na-like, and Cu-like ions. Phys. Rev. A 1993, 47, 1790-1803.

102. Träbert, E.; Beiersdorfer, P.; Lepson, J.K.; Chen, H. Extreme ultraviolet spectra of highly charged Xe ions. Phys. Rev. A 2003, 68, 042501.

103. Träbert, E.; Beiersdorfer, P.; Chen, H. Wavelengths of the $4 \mathrm{~s}_{1 / 2}-4 \mathrm{p}_{3 / 2}$ resonance lines in $\mathrm{Cu}-$ and Zn-like heavy ions. Phys. Rev. A 2004, 70, 032506. 
104. Denne, B.; Magyar, G.; Jacquinot, J. Berylliumlike Mo XXXIX and lithiumlike Mo XL observed in the Joint European Torus. Phys. Rev. A 1989, 40, 3702-3705.

105. Büttner, R.; Staude, U.; Nicolai, M.; Braukhoff, J.; Schartner, K.-H.; Folkmann, F.; Mokler, P.H. Measurement of the $2{ }^{2} \mathrm{~S}_{1 / 2}-2 \mathrm{p}^{2} \mathrm{P}_{3 / 2}$ wavelength in Li-like nickel. Nucl. Instrum. Meth. Phys. Res. B 1995, 98, 41-44.

106. Staude, U.; Bosselmann, Ph.; Büttner, R.; Horn, D.; Schartner, K.-H.; Folkmann, F.; Livingston, A.E.; Ludziejewski, T.; Mokler, P.H. Measurements of $2 \mathrm{~s}{ }^{2} \mathrm{~S}_{1 / 2}$ ï $\AA_{i} 2 \mathrm{p}{ }^{2} \mathrm{P}_{3 / 2,1 / 2}$ transition energies in lithiumlike heavy ions: Experiments and results for $\mathrm{Ni}^{25+}$ and $\mathrm{Zn}^{27+}$. Phys. Rev. A 1998, 58, 3516-3523.

107. Bosselmann, Ph.; Staude, U.; Horn, D.; Schartner, K.-H.; Folkmann, F.; Livingston, A.E.; Mokler, P.H. Measurements of $2 \mathrm{~s}^{2} \mathrm{~S}_{1 / 2}-2 \mathrm{p}{ }^{2} \mathrm{P}_{1 / 2,3 / 2}$ transition energies in lithiumlike heavy ions. II. Experimental results for $\mathrm{Ag}^{44+}$ and discussion along the isoelectronic series. Phys. Rev. A 1999, 59, 1874-1883.

108. Feili, D.; Bosselmann, Ph.; Schartner, K.-H.; Folkmann, F.; Livingston, A.E.; Träbert, E.; Ma, X.; Mokler, P.H. Measurements of $2 \mathrm{~s}^{2} \mathrm{~S}_{1 / 2}-2 \mathrm{p}{ }^{2} \mathrm{P}_{1 / 2,3 / 2}$ transition energies in lithiumlike heavy ions. III. Experimental results for $\mathrm{Sn}^{47+}$ and $\mathrm{Xe}^{51+}$. Phys. Rev. A 2000, 62, 022501.

109. Feili, D.; Bosselmann, Ph.; Schartner, K.-H.; Mokler, P.H.; Ma, X.; Livingston, A.E.; Folkmann, F; Träbert, E. Access to two-photon QED contributions via $2 \mathrm{~s}{ }^{2} \mathrm{~S}_{1 / 2}-2 \mathrm{p}{ }^{2} \mathrm{P}_{1 / 2}$ transitions in heavy Li-like ions. Phys. Scr. T 2001, 92, 300-302.

110. Schweppe. J.; Belkacem, A.; Blumenfeld, L.; Claytor, N.; Feinberg, B.; Gould, H.; Kostroun, V.E.; Levy, L.; Misawa, S.; Mowat, J.R.; et al. Measurement of the Lamb shift in lithiumlike uranium $\left(\mathrm{U}^{89+}\right)$. Phys. Rev. Lett. 1991, 66, 1434-1437.

111. Theodosiou, C.E.; Curtis, L.J.; El Mekki, M. computations of 2p Lifetimes in the Li Sequence. Phys. Rev. A 1991, 44, 7144-7157.

112. Aggarwal, K.M.; Keenan, F.P. Energy levels, radiative rates, and electron impact excitation rates for transitions in Li-like ions with $12 \leq Z \leq 20$. At. Data Nucl. Data Tables 2013, 99, 156-248.

113. Aggarwal, K.M.; Keenan, F.P. Energy levels, radiative rates, and electron impact excitation rates for transitions in Li-like ions with $21 \leq Z \leq 28$. At. Data Nucl. Data Tables 2013, 98, 1096-1108.

114. Zou, Y.; Hutton, R.; Feili, D.; Neacsu, C.; Ma, X.; Schartner, K.-H.; Mokler, P.H. Precision lifetime measurement of the $2 \mathrm{p}^{2} \mathrm{P}_{3 / 2}$ level for Li-like Ni by beam-foil spectroscopy. Nucl. Instrum. Meth. Phys. Res. B 2005, 235, 192-196.

115. Jasper, E.; Vasilyev, A.; Kukla, K.W.; Vogel Vogt, C.M.; Livingston, A.E.; Berry, H.G.; Cheng, S.; Curtis, L.J.; Dunford, R.W. Lifetime measurements for allowed and forbidden transitions. Phys. Scr. T 1999, 80, 466-468.

116. Kukla, K.W.; Livingston, A.E.; Vogel Vogt, C.M.; Berry, H.G.; Dunford, R.W.; Curtis, L.J.; Cheng, S. Extreme-ultraviolet wavelength and lifetime measurements in highly-ionized krypton. Can. J. Phys. 2005, 83, 1127-1139.

117. Epp, S.W.; Crespo López-Urrutia, J.R.; Brenner, G.; Mäckel, V.; Mokler, P.H.; Treusch, R.; Kuhlmann, M.; Yurkov, M.V.; Feldhaus, J.; Schneider, J.R.; et al. Soft X-ray laser spectroscopy on trapped highly charged ions at FLASH. Phys. Rev. Lett. 2007, 98, 183001. 
118. Träbert, E.; Armour, I.A.; Bashkin, S.; Jelley, N.A.; O’Brien, R.; Silver, J.D. The X-ray spectra of H-like, He-like and Li-like silicon ions after foil excitation. J. Phys. B 1979, 12, 1665-1676.

119. Armour, I.A.; Fawcett, B.C.; Silver, J.D.; Träbert, E. X-ray spectra and satellite classification of foil-excited Mg and Al. J. Phys. B 1980, 13, 2701-2709.

120. Träbert, E.; Fawcett, B.C.; Silver, J.D. One- and two-electron one-photon satellite transitions in the X-ray spectrum of foil-excited F and Si. J. Phys. B 1982, 15, 3587-3597.

121. Mannervik, S.; Cederquist, H.; Träbert, E. On the EUV spectrum of Li I. Phys. Scr. 1986, 34, 143-145.

122. Mannervik, S.; Short, R.T.; Sonnek, D.; Träbert, E., Möller, G.; Lodwig, V.; Heckmann, P.H.; Blanke, J.H.; Brand, K. Bound triply excited states in neutral lithium. Phys. Rev. A 1989, 39, 3964-3968.

123. Träbert, E.; Mannervik, S. Satellite lines from core-excited states in neutral lithium. Phys. Rev. A 1989, 40, 1667-1669.

124. Mannervik, S.; Cederquist, H.; Kisielinski, M. Determination of autoionization rates by means of optical emission spectroscopy. Phys. Scr. 1984, T8, 107-111.

125. Mannervik, S. Optical studies of multiply excited states. Phys. Scr. 1980, 40, 28-52.

126. Safronova, M.S.; Johnson, W.R.; Safronova, U. I Relativistic many-body computations of the energies of $n=2$ states for the berylliumlike isoelectronic sequence. Phys. Rev. A 1996, 53, 4036-4053.

127. Safronova, U.I.; Johnson, W.R.; Safronova, M.S.; Derevianko, A. Relativistic many-body computations of transition probabilities for the $2 \mathrm{l}_{1} 2 \mathrm{l}_{2}$ [LSJ] - $2 \mathrm{l}_{3} 2 \mathrm{l}_{4}$ [L'S'J'] lines in Be-like ions. Phys. Scr. 1999, 59, 286-295.

128. Safronova, U.I.; Johnson, W.R.; Derevianko, A. Relativistic many-body computations of magnetic dipole transitions in Be-like ions. Phys. Scr. 1999, 60, 46-53.

129. Chen, M.H.; Cheng, K.T. Energy levels of the ground state and the $2 \mathrm{~s} 2 \mathrm{p} \mathrm{n}(\mathrm{J}=1)$ excited states of berylliumlike ions: A large-scale, relativistic configuration-interaction computation. Phys. Rev. A 1997, 55, 166-174.

130. Möller, G.; Träbert, E.; Lodwig, V.; Wagner, C.; Heckmann, P.H.; Blanke, J.H.; Livingston, A.E.; Mokler, P.H. Experimental transition probability for the E1 intercombination transition in Be-like $\mathrm{Xe}^{50+}$. Z. Phys. D 1989, 11, 333-334.

131. Brage, T.; Fleming, J.; Hutton, R. A review of intercombination lines in beryllium-like ions. Mol. Phys. 2000, 98, 1057-1065.

132. Fawcett, B.C. Wavelengths and classifications of emission lines due to $2 \mathrm{~s}^{2} 2 \mathrm{p}^{n}-2 \mathrm{~s} 2 \mathrm{p}^{n+1}$ and $2 \mathrm{~s} 2 \mathrm{p}^{n}-2 \mathrm{p}^{n+1}$ transitions, $Z \leq 28$. At. Data Nucl. Data Tables 1975, 16, 135-164.

133. Hutton, R.; Engström, L.; Träbert, E. Experimental oscillator strengths for the resonance lines in Na- and Mg-like iron. Nucl. Instrum. Meth. Phys. Res. B 1988, 31, 294-299.

134. Cheng, K.T.; Chen, M.H.; Johnson, W.R. Hyperfine quenching of the $2 \mathrm{~s} 2 \mathrm{p}{ }^{3} \mathrm{P}_{0}$ state of berylliumlike ions. Phys. Rev. A 2008, 77, 052504.

135. Träbert, E. E1-forbidden transition rates in ions of astrophysical interest. In Proceedings of the ASOS11 Conference, Mons, Belgium, 4-9 August 2013. Phys. Scr. T 2014, submitted. 
136. Kaufman, V.; Sugar, J. Forbidden lines in $\mathrm{ns}^{2} \mathrm{np}^{k}$ ground configurations and ns np excited configurations of beryllium through molybdenum atoms and ions. J. Phys. Chem. Ref. Data 1986, $15,321-427$.

137. Huang, K.-N.; Kim, Y.-K.; Cheng, K.T.; Desclaux, J.P. Correlation and relativistic effects in spin-orbit splitting. Phys. Rev. Lett. 1982, 48, 1245-1248.

138. Safronova, M.S.; Johnson, W.R.; Safronova, U.I. Relativistic many-body computations of energies of $n=2$ states for boronlike ions. Phys. Rev. A 1996, 54, 2850-2862.

139. Merkelis, G.; Vilkas, M.J.; Gaigalas, G.; Kisielius, R. MBPT computation of energy spectra and E1 transition probabilities for boron isoelectronic sequence. Phys. Scr. 1995, 51, 233-251.

140. Vilkas, M.J.; Ishikawa, Y; Koc, K. Second-order multiconfigurational Dirac-Fock computations on boronlike ions. Int. J. Quantum Chem. 1998, 70, 813-823.

141. Li, J.-G.; Jönsson, P.; Dong, C.-Z.; Gaigalas, G. Two-electron-one-photon M1 and E2 transitions between the states of the $2 \mathrm{p}^{3}$ and $2 \mathrm{~s}^{2} 2 \mathrm{p}$ odd configurations for B-like ions with $18 \leq \mathrm{Z} \leq 92$. J. Phys. B: At. Mol. Opt. Phys. 2010, 43, 035005.

142. Safronova, U.I.; Johnson, W.R.; Livingston, A.E. Relativistic many-body computations of electric-dipole transitions between $n=2$ states in B-like ions. Phys. Rev. A 1999, 60, 996-1004; erratum 2000, 61, 039901.

143. Jönsson, P.; Li, J.-G.; Gaigalas, G.; Dong, C.-Z. Hyperfine structures, isotope shifts, and transition rates of C II, N III, and O IV from relativistic configuration interaction computations. At. Data Nucl. Data Tables 2010, 96, 271-298.

144. Rynkun, P.; Jönsson, P.; Gaigalas, G.; Froese Fischer, C. Energies and E1, M1, E2, M2 transition rates for states of the $2 \mathrm{~s}^{2} 2 \mathrm{p}, 2 \mathrm{~s} 2 \mathrm{p}^{2}$, and $2 \mathrm{p}^{3}$ configurations in boron-like ions between N III and $\mathrm{Zn}$ XXVI. At. Data Nucl. Data Tables 2012, 98, 481-556.

145. Jönsson, P.; Ekman, J.; Gustafsson, S.; Hartman, H.; Karlsson, L.B.; du Rietz, R.; Gaigalas, G.; Godefroid, M.; Froese Fischer, C. Energy levels and transition rates for the boron isoelectronic sequence: Si X, Ti XVIII - Cu XXV. Astron. Astrophys. 2013, 559, A100.

146. Träbert, E.; Heckmann, P.H.; Schlagheck, W.; Buttlar, H.V. Beam-foil lifetime studies of highly ionized silicon. Phys. Scr. 1980, 21, 27-34.

147. Träbert, E.; Heckmann, P.H. EUV spectrum and lifetimes of foil-excited phosphorus. Phys. Scr. 1980, 21, 35-39.

148. Tordoir, X.; Biémont, E.; Garnir, H.P.; Dumont, P.-D.; Träbert, E. Atomic lifetimes and transition probabilities in boron-like (Na VII) and beryllium-like (Na VIII) sodium ions. Eur. Phys. J. D 1999, 6, 1-7.

149. Doerfert, J.S.; Träbert, E. Relative intensities of $2 \mathrm{~s}^{2} 2 \mathrm{p}{ }^{2} \mathrm{P}-2 \mathrm{~s} 2 \mathrm{p}^{2}{ }^{2} \mathrm{~S},{ }^{2} \mathrm{P}$ transitions in B-like ions of oxygen through chlorine. Phys. Scr. 1993, 47, 524-530.

150. Engström, L.; Kirm, M.; Bengtsson, P.; Maniak, S.T.; Curtis, L.J.; Träbert, E., Doerfert, J.S.; Granzow, J. Extended analysis of intensity anomalies in the Al I isoelectronic sequence. Phys. Scr. 1995, 52, 516-521.

151. Träbert, E. On the transition rates of the FeX and Fe XIV coronal lines. Astron. Astrophys. 2004, 415, L39-L42. 
152. Träbert. E.; Beiersdorfer, P.; Gwinner, G.; Pinnington, E.H.; Wolf, A. M1 transition rate in $\mathrm{Cl}^{12+}$ from an electron-beam ion trap and heavy-ion storage ring. Phys. Rev. A 2002, 66, 052507.

153. Moehs, D.P.; Church, D.A. Magnetic dipole transition rates from measured lfetimes of levels of Be-like and B-like argon ions. Phys. Rev. A 1998, 58, 1111-1114.

154. Serpa, F.G.; Gillaspy, J.D.; Träbert, E. Lifetime measurements in the ground configuration of $\mathrm{Ar}^{13+}$ and $\mathrm{Kr}^{2+}$ using an electron beam ion trap. J. Phys. B: At. Mol. Opt. Phys. 1998, 31, 3345-3352.

155. Träbert, E.; Beiersdorfer, P.; Utter, S.B.; Brown, G.V.; Chen, H.; Harris, C.L.; Neill, P.A.; Savin, D.W.; Smith, A.J. Experimental M1 tansition rates of coronal lines from Ar X, Ar XIV, and Ar XV. Astrophys. J. 2000, 541, 506-511.

156. Lapierre, A.; Jentschura, U.D.; Crespo López-Urrutia, J.R.; Braun, J.; Brenner, G.; Bruhns, H.; Fischer, D.; González Martínez, A.J.; Harman, Z.; Johnson, W.R.; et al. Relativistic electron correlation, quantum electrodynamics, and the lifetime of the $1 \mathrm{~s}^{2} 2 \mathrm{~s}^{2} 2 \mathrm{p}{ }^{2} \mathrm{P}_{3 / 2}^{\mathrm{o}}$ level in boronlike argon. Phys. Rev. Lett. 2005, 95, 183001.

157. Lapierre, A.; Crespo López-Urrutia, J.R.; Braun, J.; Brenner, G.; Bruhns, H.; Fischer, D.; González-Martínez, A.J.; Mironov, V.; Osborne, C.J.; Sikler, G.; et al. Lifetime measurement of the Ar XIV $1 \mathrm{~s}^{2} 2 \mathrm{~s}^{2} 2 \mathrm{p}^{2} \mathrm{P}_{3 / 2}^{\mathrm{o}}$ metastable level at the Heidelberg electron-beam ion trap. Phys. Rev. A 2006, 73, 052507.

158. Träbert, E.; Gwinner, G.; Wolf, A.; Tordoir, X.; Calamai, A.G. Magnetic dipole transition rates in B-like and F-like titanium ions measured at a heavy-ion storage ring. Phys. Lett. A 1999, 264, 311-317.

159. Nemouchi, M.; Godefroid, M.R. Irreducible tensor fomathrm of the relativistic corrections to the M1 transition operator. J. Phys. B: At. Mol. Opt. Phys. 2009, 42, 175002.

160. Hibbert, A. Estimation of inaccuracies in oscillator strength computations. Phys. Scr. T 1996, 65, 104-109.

161. Brage, T.; Hibbert, A.; Leckrone, D.S. Transition rates of the intercombination UV0.01 multiplet in N II. Astrophys. J. 1997, 478, 423-429.

162. Träbert, E., Wolf, A.; Pinnington, E.H.; Linkemann, J.; Knystautas, E.J.; Curtis, A.; Bhattacharya, N.; Berry, H.G. Measurement of the N1 2s $2 \mathrm{p}^{3}{ }^{5} \mathrm{~S}_{2}^{o}$ level lifetime using a heavy-ion storage ring. Phys. Rev. A 1998, 58, 4449-4452.

163. Jönsson, P.; Bieroń, J. Relativistic configuration interaction computations of energy levels, isotope shifts, hyperfine structures, and transition rates in the $2 \mathrm{~s}^{2} 2 \mathrm{p}^{2}-2 \mathrm{~s} 2 \mathrm{p}^{3}$ transition array for the carbon-like sequence. J. Phys. B: At. Mol. Opt. Phys. 2010, 43, 074023.

164. Jönsson, P.; Rynkun, P.; Gaigalas, G. Energies, E1, M1, E2 transition rates, hyperfine structures, and Landé gJ factors for states of the $2 \mathrm{~s}^{2} 2 \mathrm{p}^{2}, 2 \mathrm{~s} 2 \mathrm{p}^{3}$, and $2 \mathrm{p}^{4}$ configurations in carbon-like ions between F IV and Ni XXIII. At. Data Nucl. Data Tables 2011, 97, 648-691.

165. Rynkun, P.; Jönsson, P.; Gaigalas, G.; Froese Fischer, C. Energies and E1, M1, E2, and M2 transition rates for states of the $2 \mathrm{~s}^{2} 2 \mathrm{p}^{3}, 2 \mathrm{~s} 2 \mathrm{p}^{4}$, and $2 \mathrm{p}^{5}$ configurations in nitrogen-like ions between F III and Kr XXX. At. Data Nucl. Data Tables 2013, 100, 315-402. 
166. Träbert, E.; Calamai, A.G.; Gillaspy, J.D.; Gwinner, G.; Tordoir, X.; Wolf, A. Intercombination and forbidden transition rates in $\mathrm{C}$ - and $\mathrm{N}$-like ions $\left(\mathrm{O}^{2+}, \mathrm{F}^{3+}\right.$, and $\left.\mathrm{S}^{9+}\right)$ measured at a heavy-ion storage ring. Phys. Rev. A 2000, 62, 022507.

167. Träbert, E.; Wolf, A.; Pinnington, E.H.; Linkemann, J.; Knystautas, E.J.; Curtis, A.; Bhattacharya, N.; Berry, H.G. Heavy-ion storage ring measurement of forbidden transition rates between ground-configuration levels in $\mathrm{Si}^{6+}$ and $\mathrm{Si}^{8+}$ ions. Can. J. Phys. 1998, 5876, 899-906.

168. Träbert, E.; Grieser, M.; Hoffmann, J.; Krantz, C.; Repnow; R.; Wolf, A.; Heavy-ion storage-ring-lifetime measurement of metastable levels in the $\mathrm{C}-, \mathrm{N}-$, and $\mathrm{O}$-like ions of $\mathrm{Si}, \mathrm{P}$, and S. Phys. Rev. A 2012, 85, 042508.

169. Edlén, B. Comparison of theoretical and experimental level values of the $n=2$ complex of ions isoelectronic with Li, Be, O and F. Phys. Scr. 1983, 28, 51-67.

170. Jönsson, P.; Alkauskas, A.; Gaigalas, G. Energies and E1, M1, E2 transition rates for states of the image and image configurations in fluorine-like ions between Si VI and W LXVI. At. Data Nucl. Data Tables 2013, 99, 431-446.

171. Jönsson, P.; Bengtsson, P.; Ekman, J.; Gustafsson, S.; Karlsson, L.B.; Gaigalas, G.; Froese Fischer, C.; Kato, D.; Murakami, I.; Sakaue, H.A.; et al. Relativistic CI computations of spectroscopic data for the $2 \mathrm{p}^{6}$ and $2 \mathrm{p}^{5} 31$ configurations in Ne-like ions between $\mathrm{Mg}$ III and $\mathrm{Kr}$ XXVII. At. Data Nucl. Data Tables 2014, 100, 1-154.

172. Träbert, E. Beam-foil lifetime study of Neon-like Ti ${ }^{12+}$. Z. Phys. D 1986, 1, 283-286.

173. Kirm, M.; Bengtsson, P.; Engström, L. Experimental investigation of atomic lifetimes for the $2 \mathrm{p}^{5} 31$ levels in Ne-like sulphur. Phys. Scr. 1996, 54, 167-173.

174. Träbert, E.; Jupén, C. Identification of $2 \mathrm{~s}^{2} 2 \mathrm{p}^{5} 3 \mathrm{p}{ }^{1} \mathrm{~S}_{0}$ decays in the spectra of Ne-like ions. Phys. Scr. 1987, 36, 586-590.

175. Ishikawa, Y.; López Encarnaciön, J.M.; Träbert, E. N = 3-3 transitions of Ne-like ions in the iron group, especially $\mathrm{Ca}^{10+}$ and $\mathrm{Ti}^{12+}$. Phys. Scr. 2009, 79, 025301.

176. DelZanna, G.; Ishikawa, Y. Benchmarking atomic data for astrophysics: Fe XVII EUV lines. Astron. Astrophys. 2009, 508, 1517-1526.

177. Beiersdorfer, P.; Träbert, E.; Lepson, J.K.; Brickhouse, N.S.; Golub, L. High-resolution laboratory measurements of coronal lines in the 198-218 Å region. Astrophys. J. Suppl. Ser. 2014, In print.

178. Schectman, R.M.; Curtis, L.J.; Chojnacki, D.A. Redetermination of the meanlife of the $2 p$ level in Ne I using cascade analysis. J. Opt. Soc. Am. 1973, 63, 99-100.

179. Westerlind, M.; Engström, L.; Bengtsson, P.; Curtis, L.J. Experimental lifetimes of the $2 \mathrm{p}^{5} 3 \mathrm{~s}{ }^{1} \mathrm{P}_{1}$ and ${ }^{3} \mathrm{P}_{1}$ levels in Ne-like sulfur and chlorine. Phys. Rev. A 1992, 45, 6198-6201.

180. Curtis, L.J.; Maniak, S.T.; Ghrist, R.W.; Irving, R.E.; Ellis, D.G.; Henderson, M.; Kacher, M.H.; Träbert, E.; Granzow, J.; Bengtsson, P.; Engström, L. Measurements and data-based predictions for $\Delta n=1$ resonance and intercombination transitions in the Be and Ne sequences. Phys. Rev. A 1995, 51, 4575-4582.

181. Träbert, E. Beam-foil Lifetimes of $2 \mathrm{p}^{5} 3 \mathrm{~s}{ }^{1,3} \mathrm{P}_{1}^{\mathrm{o}}$ levels in Ne-like ions of Na through S. Phys. Scr. 1996, 53, 167-173.

182. Beiersdorfer, P.; Osterheld, A.L.; Decaux, V.; Widmann, K. Observation of Lifetime-Limited X-Ray Linewidths in Cold Highly Charged Ions. Phys. Rev. Lett. 1996, 77, 5353-5356. 
183. Brown, G.V.; Beiersdorfer, P.; Widmann, K. Systematic measurement of the relative electron-impact excitation cross section of the $3 \mathrm{~d} \rightarrow 2 \mathrm{p}{ }^{1} \mathrm{P}_{1}$ resonance and ${ }^{3} \mathrm{D}_{1}$ intercombination lines in mid-Z neonlike ions. Phys. Rev. A 2001, 63, 032719.

184. Brown, G.V.; Beiersdorfer, P.; Liedahl, D.A.; Widmann, K.; Kahn, S.M.; Clothiaux, E.J. Laboratory measurements and identifications of the Fe XVIII-XXIV L-shell X-ray line emission. Astrophys. J. Suppl. Ser. 2002, 140, 589-607.

185. Gu, M.F. The flexibe atomic code. Can. J. Phys. 2008, 86, 675-689.

186. Brown, G.V.; Beiersdorfer, P. Reply to a comment. Phys. Rev. Lett. 2012, 108, 139302.

187. Bernitt, S.; Brown, G.V.; Rudolph, J.K.; Steinbrügge, R.; Graf, A.; Leutenegger, M.; Epp, S.W.; Eberle, S.; Kubicek, K.; Mäckel, V.; et al. An unexpectedly low oscillator strength as the origin of the Fe XVII emission problem. Nature 2012, 492, 225-228.

188. Cowan, T.E.; Bennett, C.L.; Dietrich, D.D.; Bixler, J.V.; Hailey, C.J.; Henderson, J.R.; Knapp, D.A.; Levine, M.A.; Marrs, R.E., Schneider, M.B. Precision measurement of the $3 \mathrm{~s}_{1 / 2}-3 \mathrm{p}_{3 / 2}$ transition energy in Na-like platinum ions. Phys. Rev. Lett. 1991, 66, 1150-1153.

189. Chen, M.H.; Cheng, K.T.; Beiersdorfer, P.; Sapirstein, J. Transition energies of the $3 \mathrm{~s}-3 \mathrm{p}_{3 / 2}$ resonance lines in sodiumlike to phosphoruslike uranium Phys. Rev. A 2003, 68, 022507.

190. Gillaspy, J.D.; Draganić,I.N.; Ralchenko, Yu.; Reader, J.; Tan, J.N.; Pomeroy, J.M.; Brewer, S.M. Measurement of the D-line doublet in high-Z highly charged sodiumlike ions. Phys. Rev. A 2009, $80,010501$.

191. Gillaspy, J.D.; Osin, D.; Ralchenko, Yu.; Reader, J.; Blundell, S.A. Transition energies of the D lines in Na-like ions. Phys. Rev. A 2013, 87, 062503.

192. Vilkas, M.J.; Ishikawa, Y.; Träbert, E. Relativistic multireference many-body perturbation theory computations on $\mathrm{Au}^{64+}-\mathrm{Au}^{69+}$ ions. Eur. Phys. J. D 2006, 41, 77-93.

193. Träbert, E.; Wolf. A.; Linkemann, J.; Tordoir, X. Optical measurement of the $\mathrm{B}^{+}$and $\mathrm{Al}^{+}$ intercombination and $\mathrm{Sc}^{12+}$ forbidden transition rates at a heavy-ion storage ring. J. Phys. B: At. Mol. Opt. Phys. 1999, 32, 537-552.

194. Johnson, B.C.; Smith, P.L.; Parkinson, W.H. Transition probability of the Al II $\lambda 2669$ intersystem line. Astrophys. J. 1986, 308, 1013-1017.

195. Träbert, E.; Hutton, R.; Martinson, I. Identification of intercombination transitions in Fe XIV and Fe XIII in the spectra of foil-excited ions and solar flares. Mon. Not. R. Astron. Soc. 1987, 227, $27 \mathrm{p}-31 \mathrm{p}$.

196. Träbert, E.; Heckmann, P.H.; Hutton, R.; Martinson, I. Intercombination lines in delayed beam-foil spectra. J. Opt. Soc. Am. B 1988, 5, 2173-2182.

197. Träbert, E.; Staude, U.; Bosselmann, P.; Schartner, K.H.; Mokler, P.H.; Tordoir, X. Intercombination transition rates in Al-like $\mathrm{Au}^{66+}$ ions. Eur. Phys. J. D 1998, 2, 117-123.

198. Santana, J.A.; Ishikawa, Y.; Träbert, E. Multireference Møller-Plesset perturbation theory results on levels and transition rates in Al-like ions of iron group elements. Phys. Scr. 2009, 79, 065301.

199. Vilkas, M.J.; Ishikawa, Y. Relativistic multireference many-body perturbation-theory computations on the multiple openshell states in siliconlike Ar and aluminumlike Fe ions. Phys. Rev. A 2003, 68, 012503. 
200. Moehs, D.P.; Bhatti, M.I.; Church, D.A. Measurements and computations of metastable level lifetimes in FeX, FeXI, FeXII, FeXIII, and Fe XIV. Phys. Rev. A 2001, 63, 032515.

201. Träbert, E.; Gwinner, G.; Wolf, A.; Knystautas, E.J.; Garnir, H.-P.; Tordoir, X. M1/E2 transition rates in Fe X through Fe XIII measured at a heavy-ion storage ring. J. Phys. B: At. Mol. Opt. Phys. 2002, 35, 671-689.

202. Träbert, E.; Calamai, A.G.; Gwinner, G.; Knystautas, E.J.; Pinnington, E.H.; Wolf, A. M1/E2/M2 decay rates in Fe VII, Fe IX, Fe X and Fe XIII measured using a heavy-ion storage ring. J. Phys. B: At. Mol. Opt. Phys. 2003, 36, 1129-1141.

203. Beiersdorfer, P.; Träbert, E.; Pinnington, E.H. Experimental transition rate of the green coronal line of Fe XIV. Astrophys. J. 2003, 587, 836-840.

204. Smith, S.J.; Chutjian, A.; Lozano, J.A. Measurement of metastable lifetimes for transitions in $\mathrm{Fe}^{9+}, \mathrm{Fe}^{10+}$, and $\mathrm{Fe}^{13+}$. Phys. Rev. A 2005, 72, 062504.

205. Brenner, G.; Crespo López-Urrutia, J.R.; Harman, Z.; Mokler, P.H.; Ullrich, J. Lifetime determination of the Fe XIV $3 \mathrm{~s}^{2} 3 \mathrm{p}^{2} \mathrm{P}_{3 / 2}^{\mathrm{o}}$ metastable level. Phys. Rev. A 2007, 75, 032504.

206. Träbert, E.; Hoffmann, J.; Krantz, C.; Wolf, A.; Ishikawa, Y.; Santana, J.A. Atomic lifetime measurements on forbidden transitions of $\mathrm{Al}-, \mathrm{Si}-, \mathrm{P}-$ and $\mathrm{S}-$ like ions at a heavy-ion storage ring. J. Phys. B: At. Mol. Opt. Phys. 2009, 42, 025002.

207. Bengtsson, P.; Ando, K.; Kambara, T.; Awaya, Y.; Hutton, R. Intercombination lines in highly charged Al- and Si-like ions. Phys. Scr. T 1997, 73, 81-82.

208. Träbert, E. Contradictory observations in beam-foil EUV spectroscopy at high ion energies. Phys. Scr. 1999, 59, 443-445.

209. Ishikawa, Y.; Vilkas, M.J.: Relativistic multireference Møller-Plesset perturbation theory computations on the term energy and lifetime of the ${ }^{5} \mathrm{~S}_{2}^{\circ}$ state in siliconlike ions with $Z=28-79$. Phys. Scr. 2002, 65, 219-226.

210. Biémont, E. Energy-level scheme and oscillator strengths for the 3s-3p and 3p-3d transitions in silicon sequence for elements vanadium through nickel. Phys. Scr. 1986, 33, 324-335.

211. Biémont, E. $3 s^{2} 3 p^{2}-3 s 3 p^{3}, 3 s^{2} 3 p^{2}-3 s^{2} 3 p 3 d$, and $3 s^{2} 3 p^{2}-3 s^{2} 3 p 4 s$ transitions in the silicon sequence for elements potassium through titanium. J. Opt. Soc. Am. B 1986, 3, 163-176.

212. Träbert, E. Solar EUV line identifications from delayed beam-foil spectra. Mon. Not. R. Astron. Soc. 1998, 297, 399-404.

213. Ishikawa, Y.; Vilkas, M.J. Relativistic multireference many-body perturbation-theory computations of the magnetic-dipole and electric-quadrupole transition probabilities of ions in the silicon isoelectronic sequence. Phys. Rev. A 2001, 63, 042506.

214. Vilkas, M.J.; Ishikawa, Y. High-accuracy computations of term energies and lifetimes of silicon-like ions with nuclear charges $Z=24-30$. J. Phys. B: At. Mol. Opt. Phys. 2004, 37, 1803-1816.

215. Träbert, E.; Ishikawa, Y; Santana, J.A.; Del Zanna, G. The $3 \mathrm{~s}^{2} 3 \mathrm{p} 3 \mathrm{~d}{ }^{3} \mathrm{~F}^{o}$ term in the Si-like spectrum of Fe (Fe XIII). Can. J. Phys. 2011, 89, 403-412.

216. Del Zanna, G. Benchmarking atomic data for astrophysics: Fe XIII. Astron. Astrophys. 2011, 533, A12. 
217. Del Zanna, G.; Storey, P.J. Atomic data for astrophysics: Fe XIII soft X-ray lines. Astron. Astrophys. 2012, 543, A144.

218. Froese Fischer, C. Towards accurate transition data for $3 \mathrm{p}^{2}, 3 \mathrm{p}^{3}, 3 \mathrm{p}^{4}$ levels of Fe, Co and Ni ions. J. Phys. B: At. Mol. Opt. Phys. 2010, 43, 074020.

219. Mendoza, C.; Zeippen, C.J. Transition probabilities for forbidden lines in the $3 \mathrm{p}^{2}$ configuration-II. Mon. Not. R. Astron. Soc. 1982, 199, 1025-1032.

220. Abou El-Maaref, A.; Uosif, M.A. M.; Allam, S.H.; El-Sherbini, Th. M. Energy levels, oscillator strengths and transition probabilities for Si-like P II, S III, Cl IV, Ar V and K VI. At. Data Nucl. Data Tables 2012, 98, 589-615.

221. Abou El-Maaref, A.; Allam, S.H.; El-Sherbini, Th.M. Energy levels, oscillator strengths and radiative rates for Si-like Zn XVII, Ga XVIII, Ge XIX, ans As XX. At. Data Nucl. Data Tables 2014, 100, 155-182.

222. Vilkas M.J.; Ishikawa Y. Relativistic many-body perturbation computations on extreme ultraviolet and soft-x-ray transition energies in siliconlike iron. Phys. Rev. A 2004, 69, 062503.

223. Mendoza, C.; Zeippen, C.J. Transition probabilities for forbidden lines in the $3 \mathrm{p}^{3}$ configuration. Mon. Not. R. Astron. Soc. 1982, 198, 127-139.

224. Biémont, E.; Hansen, J.E. Radiative transition rates in the ground configuration of the phosphorus sequence from argon to ruthenium. Phys. Scr. 1985, 31, 509-518.

225. Biémont, E.; Palmeri, P.; Quinet, P.; Träbert, E.; Zeippen, C.J. Level lifetimes in 3p and 3d configurations of Fe XII. Eur. Phys. J. D 2002, 20, 37-44.

226. Vilkas, M.J.; Ishikawa Y. Relativistic multireference many-body perturbation theory calculations on ions of the phosphorus isoelectronic sequence. J. Phys. B: At. Mol. Opt. Phys. 2004, 37, 4763-4778.

227. Del Zanna, G.; Mason, H.E. Benchmarking atomic data for astrophysics: Fe XII. Astron. Astrophys. 2005, 433, 731-744.

228. Mendoza, C.; Zeippen, C.J. Transition probabilities for forbidden lines in the $3 \mathrm{p}^{4}$ configuration-III. Mon. Not. R. Astron. Soc. 1983, 202, 981-986.

229. Biémont, E.; Hansen, J.E. Forbidden transitions in $3 \mathrm{p}^{4}$ and $4 \mathrm{p}^{4}$ configurations. Phys. Scr. 1986, 34, 116-130.

230. Saloman, E.B.; Kim, Y.-K. Energy levels and transition probabilities in the ground-state configuration of sulfur-like ions. At. Data Nucl. Data Tables 1989, 41, 339-356.

231. Chou, H.-S.; Chang, J.-Y.; Chang, Y.-H.; Huang, K.-N. Energy-level scheme and transition probabilities of S-like ions. At. Data Nucl. Data Tables 1996, 62, 77-145.

232. Ishikawa, Y.; Vilkas, M.J. Relativistic many-body computations of excited-state energies and transition wavelengths for six-valence-electron sulfurlike ions. Phys. Rev. A 2008, 78, 042501.

233. Del Zanna, G. Benchmarking atomic data for astrophysics: Fe XI. Astron. Astrophys. 2010, 514, A41.

234. Del Zanna, G.; Storey, P.J. Atomic data for astrophysics: Fe XI soft X-ray lines. Astron. Astrophys. 2013, 549, A42.

235. Biémont, E.; Träbert, E. Transition rates of the resonance line doublet in the $\mathrm{Cl}$ I sequence, Ar II-Ge XVI. J. Phys. B: At. Mol. Opt. Phys. 2000, 33, 2939-2946. 
236. Del Zanna, G.; Berrington, K.A.; Mason, H.E. Benchmarking atomic data for astrophysics: Fe X. Astron. Astrophys. 2004, 422, 731-749.

237. Del Zanna, G.; Berrington, K.A.; Mason, H.E. Atomic data for astrophysics: Fe X soft X-ray lines. Astron. Astrophys. 2012, 541, A90.

238. Ishikawa, Y.; SAntana, J.A.; Träbert, E. Relativistic multireference many-body perturbation theory for open-shell ions with multiple valence shell electrons: the transition rates and lifetimes of the excited levels in chlorinelike Fe X. J. Phys. B: At. Mol. Opt. Phys. 2010, 43, 074022.

239. Träbert, E.; Saathoff, G.; Wolf, A. M1/E2 decay rates in Co XI, Ni XII and Cu XIII measured at a heavy-ion storage ring. Can. J. Phys. 2004, 37, 945-952.

240. Träbert, E. Experimental checks on computations for Cl-, S- and P-like ions of the iron group elements. J. Phys. B: At. Mol. Opt. Phys. 1996, 29, L217-L224.

241. Dumont, P.D.; Garnir, H.P.; Baudinet-Robinet, Y.; Kapenyak, M. Lifetime measurements in Ti IV-VII using transitions observed in beam-foil spectroscopy between 400 and $800 \AA$. J. Opt. Soc. Am. 1981, 71, 502-503.

242. Mohan, M.; Singh, A.K.; Jha, A.K. S.; Jha, P. Level energies, oscillator strengths, and lifetimes for transitions in Ti VI. At. Data Nucl. Data Tables 2007, 93, 105-126.

243. Garnir, H.P.; Dumont, P.D.; Tordoir, X. Atomic lifetime measurement of K III. unpublished work.

244. Lauer, S.; Liebel, H.; Vollweiler, F.; Schmoranzer, H.; Lagutin, B.M.; Demekhin, Ph.V.; Petrov, I.D.; Sukhorukov, V.L. Lifetimes of the $\mathrm{ns}^{1} \mathrm{np}^{6}{ }^{2} \mathrm{~S}_{1=2}$ states of singly ionized argon, krypton and xenon. J. Phys. B: At. Mol. Opt. Phys. 1999, 32, 2015-2030.

245. Wilson, N.J.; Hibbert, A.; Bell, K.L. Oscillator Strengths for K III, Ca IV and Sc V. Phys. Scr. 2000, 61, 603-610.

246. Berrington, K.A.; Pelan, J.C.; Waldock, J.A. Oscillator strength for $3 s^{2} 3 p^{5}-3 s 3 p^{6}$ in Cl-like ions. J. Phys. B: At. Mol. Opt. Phys. 2001, 34, L419-L424.

247. Saha, B.; Fritzsche, S. M1 and E2 transitions in Ar II. J. Phys. B: At. Mol. Opt. Phys. 2005, 38, 1161-1171.

248. Wagner, W.J.; House, L.L. Hartree-Fock computations of coronal forbidden lines in the Argon I isoelectronic sequence. Astrophys. J. 1969, 155, 677-686.

249. Wagner, W.J.; House, L.L. Emprically corrected computations of coronal visible lines from the $3 \mathrm{p}^{5} 3 \mathrm{~d}$ configuration. Astrophys. J. 1971, 166, 683-698.

250. Träbert, E.; Jupén, C.; Fritzsche, S. EUV line identifications and lifetime measurements in highly-charged ions of the iron group. Phys. Scr. T 1999, 80, 463-465.

251. Träbert, E.; Beiersdorfer, P.; Brown, G.V.; Chen, H.; Thorn, D.B.; Biémont, E. Experimental M1 transition rates in highly charged Kr ions. Phys. Rev. A 2001, 64, 042511.

252. Utter, S.B.; Beiersdorfer, P.; Träbert, E.; Clothiaux, E.J. Wavelengths of the $4 \mathrm{~s}_{1 / 2}-4 \mathrm{p}_{3 / 2}$ resonance lines in Cu-like heavy ions. Phys. Rev. A 2003, 67, 032502.

253. Vilkas, M.J.; Ishikawa, Y. Relativistic multireference many-body perturbation computations on multi-valence-electron systems: Benchmarks on Zn-like ions. Phys. Rev. A 2005, 72, 032512.

254. Ishikawa, Y.; Quiney, H.M.; Malli, G.L. Dirac-Fock-Breit self-consistent-field method: Gaussian basis-set computations on many-electron atoms. Phys. Rev. A 1991, 43, 3270-3278. 
255. Malli, G.L.; Da Silva, A.B.F.; Ishikawa, Y. Universal Gaussian basis set for accurate ab initio relativistic Dirac-Fock computations. Phys. Rev. A 1993, 47, 143-146.

256. Blundell, S.A.; Johnson, W.R.; Safronova, M.S.; Safronova, U.I. Relativistic many-body computations of the energies of $n=4$ states along the zinc isoelectronic sequence. Phys. Rev. A 2008, 77, 032507.

257. Blundell, S.A. computations of QED corrections in highly charged Zn-like ions. Can. J. Phys. 2009, 87, 55-65.

258. Safronova, U.I.; Safronova, M.S. Relativistic many-body computations of the oscillator strengths, transition rates and polarizabilities in Zn-like ions. J. Phys. B: At. Mol. Opt. Phys. 2010, 43, 074025.

259. Chen, M.H.; Cheng, K.T. A large-scale relativistic configuration-interaction approach: application to the $4 s^{2}-4 s 4 p$ transition energies and E1 rates for Zn-like ions. J. Phys. B: At. Mol. Opt. Phys. 2010, 43, 074019.

260. Träbert, E.; Clementson, J.; Beiersdorfer, P.; Santana, J.A.; Ishikawa, Y. Measurements and computations of Zn-like heavy ions: an update. Can. J. Phys. 2011, 89, 639-645.

261. Träbert, E.; Clementson, J.; Beiersdorfer, P.; Santana, J.A.; Ishikawa, Y. Extreme-ultraviolet spectra of highly charged Pt ions with several valence-shell electrons: Observation and accurate computations. Phys. Rev. A 2010, 82, 062519.

262. Santana, J.A.; Ishikawa, Y.; Träbert, E. Relativistic MR-MP computations of the energy levels and transition probabilities in Ni- to Kr-like Pt ions. At. Data Nucl. Data Tables 2014, 100, 183-271.

263. Utter, S.B.; Beiersdorfer, P.; Träbert, E. Electron-beam ion-trap spectra of tungsten in the EUV. Can. J. Phys. 2002, 80, 1503-1515.

264. Träbert, E. EUV beam-foil observations of $\mathrm{Cu}$-like ions through Ge-like ions of iodine. Phys. Scr. T 2011, 144, 014004.

265. Li, J.G.; Träbert, E.; Dong, C.Z. Energy levels, transition rates and lifetimes for low-lying levels in $\mathrm{Cu}-, \mathrm{Zn}-, \mathrm{Ga}-$ and Ge-like ions of iodine. Phys. Scr. 2011, 83, 015301.

266. Curtis, L.J.; Ellis, D.G. Alkali Spectra in the Promethium Isoelectronic Sequence. Phys. Rev. Lett. 1980, 45, 2099-2102.

267. Träbert, E.; Heckmann, P.H. Tentative Identification of the 5s-5p Transitions in Pm I-Like Au XIX. Z. Phys. D 1986, 1, 381-383.

268. Kaufman, V.; Träbert, E.; Heckmann, P.H.; Möller, G.; Lodwig, V.; Blanke, J.H. Search for intercombination lines in few-electron spectra of rare-earth sequence ions of Os through Au. Phys. Scr. 1990, 42, 705-713

269. Vilkas, M.J.; Ishikawa, Y.; Träbert, E. Electric-dipole $5 \mathrm{~s}-5 \mathrm{p}$ transitions in promethiumlike ions. Phys. Rev. A 2008, 77, 042510.

270. Kobayashi, Y.; Kato, D.; Sakaue, H.A.; Murakami, I.; Nakamura, N. Spectroscopic study of promethiumlike bismuth with an electron-beam ion trap: Search for alkali-metal-like resonance lines Phys. Rev. A 2014, 89, 010501.

271. Kramida, A.E.; Shirai, T. Energy levels and spectral lines of tungsten, W III through W LXXIV. At. Data Nucl. Data Tables 2009, 95, 305-474; Erratum: 2009, 95, 1051. 
272. Beiersdorfer, P.; Osterheld, A.L.; Scofield, J.; Wargelin, B.; Marrs, R.E. Observation of magnetic octupole decay in atomic spectra. Phys. Rev. Lett. 1991, 67, 2272-2275.

273. Clementson, J.; Beiersdorfer, P.; Brown, G.V.; Gu, M.F. Spectroscopy of M-shell x-ray transitions in Zn-like through Co-like W. Phys. Scr. 2010, 81, 015301.

274. Clementson, J.; Beiersdorfer, P. Wavelength measurement of $n=3$ to $n=3$ transitions in highly charged tungsten ions. Phys. Rev. A 2010, 81,052509.

275. Clementson, J.; Beiersdorfer, P.; Brown, G.V.; Gu, M.F.; Lundberg, H.; Podpaly, Y.; Träbert, E. Tungsten spectroscopy at the Livermore electron beam ion trap facility. Can. J. Phys. 2011, 89, 571-580.

276. Clementson, J.; Beiersdorfer, P.; Gu, M.F. X-ray spectroscopy of E2 and M3 transitions in Ni-like W. Phys. Rev. A 2010, 81, 052509.

277. Clementson, J.; Beiersdorfer, P.; Brage. T.; Gu, M.F. Atomic data and theoretical X-ray spectra of Ge-like through V-like W ions. At. Data Nucl. Data Tables 2014, 100 577-649.

278. Träbert, E.; Beiersdorfer, P.; Pinnington, E.H.; Utter, S.B.; Vilkas, M.J.; Ishikawa, Y. Experiment and theory in interplay on high-Z few-electron ion spectra from foil-excited ion beams and electron beam ion traps. J. Phys.: Conf. Ser. 2007, 58, 93-96.

279. Kramida, A.; Ralchenko, Yu.; Reader, J.; NIST ASD Team. NIST Atomic Spectra Database (ver. 5.1), 2013. National Institute of Standards and Technology, Gaithersburg, MD, USA. Available online: http://physics.nist.gov/asd (accessed on 3 January 2014).

280. Dere, K.P.; Landi, E.; Young, P.R.; Del Zanna, G.; Landini, M.; Mason, H.E. CHIANTI-An atomic database for emission lines IX. Ionization rates, recombination rates, ionization equilibria for the elements hydrogen through zinc and updated atomic data. Astron. Astrophys. 2009, 498, 915-929.

281. Landi, E.; Del Zanna, G.; Young, P.R.; Dere, K.P.; Mason, H.E. CHIANTI-An atomic data base for emission lines. XII. Version 7 of thedatabase. Astrophys. J. 2012, 744, 99.

282. Mäckel, V.; Klawitter, R.; Brenner, G.; Crespo López-Urrutia, J.R.; Ullrich, J. Laser spectroscopy on forbidden transitions in trapped highly charged $\mathrm{Ar}^{13+}$ ions. Phys. Rev. Lett. 2011, 107, 143002.

(c) 2014 by the author; licensee MDPI, Basel, Switzerland. This article is an open access article distributed under the terms and conditions of the Creative Commons Attribution license (http://creativecommons.org/licenses/by/3.0/). 